\title{
4D higher spin black holes with nonlinear scalar fluctuations
}

\author{
Carlo Iazeolla ${ }^{a}$ and Per Sundell ${ }^{b}$ \\ ${ }^{a}$ NSR Physics Department, G. Marconi University, \\ via Plinio 44, Rome, Italy \\ ${ }^{b}$ Departamento de Ciencias Físicas, Universidad Andres Bello, \\ Republica 220, Santiago de Chile \\ E-mail: c.iazeolla@gmail.com, per.anders.sundell@gmail.com
}

ABSTRACT: We construct an infinite-dimensional space of solutions to Vasiliev's equations in four dimensions that are asymptotic to AdS spacetime and superpose massless scalar particle modes over static higher spin black holes. Each solution is obtained by a large gauge transformation of an all-order perturbatively defined particular solution given in a simple gauge, in which the spacetime connection vanishes, the twistor space connection is holomorphic, and all local degrees of freedom are encoded into the residual twistor space dependence of the spacetime zero-forms. The latter are expanded over two dual spaces of Fock space operators, corresponding to scalar particle and static black hole modes, equipped with positive definite sesquilinear and bilinear forms, respectively. Switching on an AdS vacuum gauge function, the twistor space connection becomes analytic at generic spacetime points, which makes it possible to reach Vasiliev's gauge, in which Fronsdal fields arise asymptotically, by another large transformation given here at first order. The particle and black hole modes are related by a twistor space Fourier transform, resulting in a black hole backreaction already at the second order of classical perturbation theory. We speculate on the existence of a fine-tuned branch of moduli space that is free from black hole modes and directly related to the quasi-local deformed Fronsdal theory. Finally, we comment on a possible interpretation of the higher spin black hole solutions as black-hole microstates.

KeYwords: Higher Spin Gravity, Higher Spin Symmetry, Black Holes

ArXIV EPRINT: 1705.06713 


\section{Contents}

1 Introduction $\quad 1$

1.1 Motivations 1

1.2 Black holes or black-hole microstates? 3

$\begin{array}{lll}1.3 & \text { Summary of our new results } & 7\end{array}$

1.4 Plan of the paper 9

2 Vasiliev's four-dimensional bosonic models 9

2.1 Correspondence space $\quad 9$

$\begin{array}{lll}2.2 & \text { Master fields } & 15\end{array}$

$\begin{array}{ll}2.3 \text { Equations of motion } & 17\end{array}$

3 Solution method $\quad 21$

3.1 Gauge functions 21

3.2 Separation of variables in twistor space and holomorphicity 24

4 Internal solution space with scalar field and black hole modes $\quad 27$

$\begin{array}{lll}4.1 & \text { Particle and black hole modes } & 27\end{array}$

$\begin{array}{lll}4.2 & \text { Regular presentation of generalized projectors } & 29\end{array}$

$\begin{array}{lll}4.3 & \text { Explicit form of internal solution in holomorphic gauge } & 30\end{array}$

$\begin{array}{lll}4.4 & \text { Black-hole backreaction from scalar particle modes } & 35\end{array}$

$\begin{array}{lll}\text { 4.5 } & \text { Embedding into an associative algebra } & 37\end{array}$

5 Spacetime dependence of the master fields $\quad 39$

$\begin{array}{lll}5.1 \text { Gauge functions } & 39\end{array}$

5.2 Master fields in $L$-gauge and Kruskal-like gauge 42

5.2.1 Weyl zero-form 42

5.2.2 Internal connection in $L$-gauge 44

$\begin{array}{lll}5.2 .3 & \text { Internal connection in Kruskal-like gauge } & 47\end{array}$

$\begin{array}{lll}5.3 & \text { Vasiliev gauge } & 48\end{array}$

6 Conclusions and outlook $\quad 51$

$\begin{array}{ll}\text { A Spinor conventions and } \boldsymbol{A d S}_{4} \text { background } & 57\end{array}$

$\begin{array}{ll}\text { B Properties of inner Klein operators } & 60\end{array}$

C Deformed oscillators with delta function Klein operator $\quad 61$

$\begin{array}{lll}\text { C.1 Problem setting } & 61\end{array}$

$\begin{array}{lll}\text { C.2 Integral equation } & 62\end{array}$

$\begin{array}{lll}\text { C.3 Solution using symbol calculus } & 64\end{array}$

$\begin{array}{lll}\text { C.4 Non-trivial vacuum connections on } \mathcal{Z} & 66\end{array}$ 


\section{Introduction}

\subsection{Motivations}

Higher spin gravities are extensions of ordinary gravity theories by massless fields with spin greater than two, based on the gauge principle. Remarkably, these assumptions lead to highly constrained interactions governed by non-abelian higher spin symmetry algebras, whose consistency requires special matter sectors and non-vanishing cosmological constant. The resulting framework thus provides a natural platform for studying tensionless string theory in anti-de Sitter spacetime [21-23] (for recent advances, see [24-26]) and holography [21, 27-41] (for a different approach, see also [42]), without relying on any a priori assumptions on dualities between strong and weak coupling on the worldsheet or the conformal field theory side.

One of the outstanding problems is to connect two different approaches to higher spin gravity that are presently pursued: Vasiliev's theory and the quasi-local deformed Fronsdal theory. The latter approach (see [43-58] and references therein; see also [59] for a review and more references, and [109-113] for more recent works on the holographic reconstruction of bulk vertices) provides a perturbatively defined deformation of the free Fronsdal action following the Noether procedure supplemented by weak locality conditions - as to ensure well-defined amplitudes built using Green's functions in anti-de Sitter spacetime with proper boundary conditions. Up to quartic order, this approach has so far been yielding holographic correlation functions corresponding to unitary free conformal field theories. As for exploring the moduli space of the theory, including more nontrivial field theory duals, the deformed Fronsdal theory has so far been of limited use, however, in the absence of any fully nonlinear completion of the Noether procedure.

Vasiliev's equations [4-8] (for reviews, focusing on various aspects of the subject, see [9-14]), on the other hand, provide a fully nonlinear classical theory based on differential graded algebras defined on noncommutative Poisson manifolds, given locally by direct products of a commuting spacetime manifold and internal symplectic manifolds. This formalism encodes the deformation of the Fronsdal equations on-shell into manifestly Cartan-integrable curvature constraints, at the expense, however, of blurring the spacetime quasi-locality, and of introducing additional moduli entering via the internal connection.

It is therefore highly non-trivial to establish whether and how the deformed Fronsdal theory and Vasiliev's formulation are connected beyond the linearized approximation. To this end, one may envisage two quite different routes, depending on whether one expects to make contact directly at the level of the spacetime action, or, more indirectly, at the level of on-shell actions subject to boundary conditions. The former path, whereby the internal degrees of freedom of Vasiliev's integrable system are viewed essentially as auxiliary fields, without any proper dynamics of their own, involves a reduction down to a set of deformed Fronsdal equations on the spacetime submanifold $[4,7-9,13,30,84,92-95]$, to be directly compared with those of the quasi-local deforrmed Fronsdal theory. Although this procedure introduces ambiguities concerning the embedding of spacetime into the full noncommutative geometry and the internal gauge fixing, one may entertain the idea that the quasi-locality requirement provides a guiding principle; for recent progress, see [95-97]. 
Following the second route, one instead seeks to implement the action principle by deriving Vasiliev's equations from a globally defined Lagrangian density on the full noncommutative manifold [15-17, 102], which requires a choice of topology and a suitable class of functions forming a differential graded algebra equipped with a trace operation and a hermitian conjugation. Making contact between the two approaches then consists of seeking up a point in moduli space leading to an on-shell action that agrees with that of the deformed Fronsdal theory. Note that this does not require any equivalence at the level of vertices and Green's functions.

To pursue the comparison, the access to explicit classical solution spaces of Vasiliev's equations may provide useful guidance, whether to extract a quasi-local Fronsdal branch of moduli space, or to compute on-shell actions on the full noncommutative geometry. So far, the classical moduli space has been explored in the direction of asymptotically anti-de Sitter solutions with special higher spin Weyl curvatures: instanton-like solutions, higher spin black holes and other generalized Type-D solutions [1-3, 66, 67, 90, 91]; see also $[63,64,101]$ for exact solutions in three spacetime dimensions, and [89] for solutions obtained in axial gauge in twistor space.

The study of exact solutions of higher-spin gravity is relevant for addressing other important open questions: for instance, as the theory is supposed to describe an "extremely stringy" extension of (super)gravity theories, a natural question to investigate is whether it admits black hole solutions, and how the main problems that their physics poses are framed in its language, and, possibly, resolved by the coupling of the gravitational fields with an infinite tower of massless fields of higher spin. Black-hole-like solutions of the Vasiliev equations have been constructed in [1-3, 91], but the present lack of a stringy generalization of geometry has so far limited our capability to assess their precise nature - for instance by establishing whether they possess a horizon, whether their curvature singularity is physical or not, etc. Moreover, whereas the Type-D solution spaces in ordinary gravity are finite-dimensional, it was observed in $[1,2]$ that their higher-spin counterparts are infinite-dimensional. We are inclined to interpret this fact, together with other properties, as an indication that such solutions are candidates for the microscopic description of hairy quantum black holes, rather then higher-spin black holes, as we shall comment on in more detail throughout this paper. Clearly, one expects that the study of fluctuations over such black-hole-like solutions should help in answering some of the key physical questions above.

In this paper, we shall extend the higher spin black hole systems of $[1,2]$ by massless scalar particle and anti-particle modes. ${ }^{1}$ The resulting configurations describe nonlinear propagating scalar fluctuation fields on static spherically-symmetric higher spin black hole backgrounds. Although these restrictions on the boundary conditions lead to technical simplifications, they do not imply any loss of generality concerning our main conclusions, as the solution method developed in this paper facilitates the systematic fully nonlinear completion of linear combinations of black-hole-like and particle modes, and therefore lends itself equally well to the construction of solution spaces consisting of general time-dependent

\footnotetext{
${ }^{1} \mathrm{~A}$ corollary of our analysis is that it is possible to map Weyl-ordered master fields on-shell to normalordered dittos obeying the central on-mass-shell theorem in the scalar field sector, as required for the embedding of the Vasiliev system into the Frobenius-Chern-Simons model on-shell [107].
} 
black hole modes superposed with general massless particle modes of any spin, which we leave for a future work.

Moreover, as we shall comment more in detail later on, the study in this paper already offers some results that may be relevant to the comparison of the two approaches to higher spin gravity. Indeed, the quasi-local deformed Fronsdal theory by construction builds a perturbation theory in which the fundamental massless particles are stable to any order which is indeed a necessary condition for it to be a candidate bulk dual of free conformal theories. At the classical level, this requires sufficiently local vertices; in particular, it requires that the result of two massless particles modes entering a cubic vertex can be expanded in terms of the same type of mode functions. On the other hand, in the Vasiliev system, which is a set of zero-curvature constraints on a higher-dimensional fibered space, the particle modes are encoded into specific fiber functions, and the classical perturbation theory is generated by applying homotopy contractors to initial data given by such elements. Thus, the issue of whether the system admits any perturbative expansion scheme describing self-interacting particles requires a careful analysis as in the fully non-linear theory there exists a vertex that interchanges particle and black hole modes. Indeed, the exact solutions of the present paper will show that, at least in certain gauges, particle modes interact to form black holes modes already at second order of classical perturbation theory — which can possibly be seen as a manifestation of the non-locality of the Vasiliev equations at each perturbative order. The special internal gauge we start from is connected by a large gauge transformation to Vasiliev's (internal) gauge, in which Fronsdal fields arise at the linearized level. Thus, aiming a direct correspondence between the Vasiliev theory and the quasi-local deformed Fronsdal theory, one may ask whether the ambiguity residing in Vasiliev's gauge beyond the linearized level can be exploited to cancel out the black hole modes at higher orders in perturbation theory, as to fine-tune to a quasi-local branch of Vasiliev's theory. We shall not pursue this refined boundary value problem in this paper, nor its analog in the alternative perturbative scheme employed by Vasiliev in his original work, though we shall remark on these topics towards the end of the paper.

\subsection{Black holes or black-hole microstates?}

The solutions to four-dimensional higher spin gravity that are referred to as higher spin black holes are distinct by a tower of electric or magnetic Weyl tensors of all spins that include and generalize the spin-2 Weyl tensor of an AdS Schwarzschild black hole. In particular, all Weyl tensors share the Killing symmetries, Petrov type D and principal spinors of the latter; ${ }^{2}$ see [3] for the first instance of such a solution, and [1, 2, 91] for developments. In gravity, such a form of the Weyl tensor is a local hallmark of a black-hole solution [68]. However, in higher spin gravity this identification is subtler: so far there is no known higher-spin

\footnotetext{
${ }^{2}$ The spin-two Weyl tensor is of Petrov Type $\mathrm{D}$, that is, $\left.C_{\alpha \beta \gamma \delta}=f u_{(\alpha} u_{\beta} v_{\gamma} v_{\delta}\right)$ where $\left(u_{\alpha}, v_{\beta}\right)$ is a spin-frame, normalized such that $u^{\alpha} v_{\alpha}=1$, and $f$ is a complex function (which remains invariant under redefinitions $u_{\alpha} \rightarrow \lambda u_{\alpha}$ and $v_{\alpha} \rightarrow \lambda^{-1} v_{\alpha}$, with $\lambda \in \mathbb{C}^{\prime}$ ). See appendix A. In particular, black-hole Weyl tensors are distinguished, in gravity, by the fact that $u_{\alpha}$ and $v_{\alpha}$ are eigenspinors of the (self-dual part of the) Killing two-form of a time-like Killing vector. In the case of a static and spherically-symmetric Type D solution, the function $f \sim \mathcal{M}_{2} r^{-3}$ in the asymptotic region, where $\mathcal{M}_{2}$ is the spin-two charge, which is real in the electric case and purely imaginary in the magnetic case.
} 
invariant quantity ensuring that the singularity of the individual Weyl tensors is physical, and whether there exists any invariant notion of an event horizon - and whether an entropy could be attached to it $^{3}$ - remains an open problem. Although there exist a large number of formally defined metrics in higher spin gravity, as (non-abelian) higher spin symmetries mix different spins as well as numbers of derivatives (in units of the cosmological constant) none of them is a priori preferred in the absence of any metric-like action principle (though calibrations of areas may select special metrics related to brane actions [88]).

On the other hand, as we shall describe more in detail below, the solutions found in $[1,2]$ form an infinite-dimensional unitarizable higher-spin module of states (the norm being given by the ordinary supertrace), each one giving rise to a solution that has identical black-hole asymptotics but that is possibly non-singular and horizon-free, which would rather suggest an interpretation in terms of black-hole microstates. In fact, viewing higher spin gravity as gravity coupled to higher spin gauge fields, it is tempting to view such solutions as higher-spin analogs of fuzzballs [69-73], that we think of as solutions that are degenerate with the AdS-Schwarzschild black hole to a (macroscopic) observer in the asymptotic region, but that contain "hairs" that modify the strongly coupled region of the black hole geometry as to create a smooth solution without a horizon. We hope to investigate the details of this proposal in a future work.

Reasoning physically, we find it plausible to entertain the idea that entropic horizons emerge in broken phases of higher spin gravity, whereas the microstates are directly visible in the unbroken phase as a spectrum of regular solutions. Indeed, the breaking of higher spin symmetries would introduce a mass scale that could give rise to Yukawa-like potentials that screen the higher spin hairs for the asymptotic observer, while higher spin gauge fields extend into the asymptotic region in the unbroken phase. Moreover, the blackhole-like solutions in Vasiliev's theory can be thought of as being smooth at the origin despite the fact that each individual Weyl tensor blows up at this point. Indeed, all Weyl tensors and auxiliary fields enter the Vasiliev equations packed into master fields living on an extension of spacetime by a noncommutative twistor space and satisfying a deformed oscillator algebra: in these terms, a sign that the origin is not a special point is that the deformed oscillator algebra remains well-defined there, even though the deformation is given by a distribution in twistor space. ${ }^{4}$

\footnotetext{
${ }^{3}$ The conservative notion of an entropy, namely as a density of states at the saddle point of a path integral, requires the further notions of an on-shell action $[15,19,102,107]$ and either a conserved set of macroscopic charges at infinity, or, alternatively, a conjugate (temperature) variable related to thermal fluctuations and Hawking radiation. Alternatively, and more formally, one may think of the entropy and the on-shell action as elements in the on-shell de Rham cohomology in spacetime form degree two and four; indeed, in [88] a structure group was found that yields a unique complex cohomology element for every strictly positive even degree (and vanishing cohomology for every positive odd degree). In [102, 103], cohomologies in degrees two and four have been constructed using different methods. See also [103] for the definition of generalized asymptotic charges.

${ }^{4}$ The global AdS radial coordinate $r$ appears in the black-hole solutions as the parameter of a delta sequence: away from the origin one has smooth Gaussian functions, approaching a Dirac delta function as $r$ goes to zero [1]. But unlike the delta function on a commutative space, which is singular thought of as an element in a ring of sections, the delta function in noncommutative twistors space is smooth thought of as a symbol for an element of a star product algebra. Indeed, one can show [1] that by changing ordering
} 
In order to stress this point, let us recall a few basic facts of the Vasiliev equations (leaving further details for section 2). Conceptually speaking, the equations are analogs of the constraints on the super-torsion and super-Riemann tensor in supergravity, in the sense that, rather than working directly with Fronsdal fields, the formalism employs a frame field, a Lorentz connection and an infinite tower of higher spin analogues. These are introduced together with a corresponding tower of zero-forms, altogether forming a Cartan integrable system, i.e. a set of curvature constraints that can be used to express all fields on shell in terms of (large) gauge functions and zero-form integration constants. Although it shares plenty of features with topological field theory, the formalism makes use of infinitely many fields, making it capable of describing systems with local degrees of freedom, which enter via infinite-dimensional spaces of integration constants, constituting a module for the higher spin Lie algebra, known as the Weyl zero-form module, or as the twisted-adjoint representation. Its Lorentz covariant basis define the generalized Weyl tensors (including spin-0 and spin- $1 / 2$ matter fields), and all their covariant derivatives on-shell at some point of the base manifold, while its compact basis describes particles, black holes, solitons and other states of the theory $[1,87]$.

The key to Vasiliev's fully nonlinear theory is the fact that both the higher spin algebra and its zero-form module arise as subspaces within one and the same associative algebra, given by sets of functions on a non-commutative twistor fiber space. In order to couple the connection to the Weyl zero-form, Vasiliev extended spacetime with an additional non-commutative twistor base space, supporting remarkable closed and twisted-central two-forms whose star products with the Weyl zero-form serve as nontrivial sources for the two-form curvature in twistor space. The resulting system thus describes a flat connection and a covariantly constant zero-form in spacetime coupled to a set of deformed oscillators in twistor space. This system can be treated in two dual fashions, by either reducing it perturbatively to deformed set of Fronsdal fields on spacetime [4, 7, 9, 84, 92], or expressing the fields using a gauge function (i.e. a large gauge transformation) and a set of deformed oscillators constructed using algebraic methods [1, 9, 66, 67, 85, 87, 91].

In a previous work [1] (see also [2, 91]), we have used the latter method to construct families of exact generalized Petrov Type D solutions, encompassed by an Ansatz based on factorization of noncommutative fiber and base twistor coordinates, absorbed into generalized projectors and deformed oscillators, respectively. The various families are distinguished by different Killing symmetries, while all share the Kerr-Schild property, i.e., the full Weyl zero-form coincides with the linearized one (in a special gauge). Thus, the Weyl zero-form can be assigned a linear space, while the twistor space connection contains interference terms (that can be removed as well in certain gauges [3] though they must be switched on eventually in going to Vasiliev's gauge).

In particular, the spherically-symmetric solutions are expanded over "skew-diagonal" projectors $\widetilde{\mathcal{P}}_{n} \sim|n / 2\rangle\langle-n / 2|$, that we shall refer to as twisted projectors, with real or

prescription (from Weyl ordering to normal ordering) one can map the delta function to a regular element, solve the equations in this basis and then move back to the original ordering — generating the same solution that one would have obtained by solving directly the deformed oscillator algebra with the distributional deformation. In this sense we can say that the black-hole-like solutions are actually smooth. 
imaginary expansion coefficients $\nu_{n}$, where $n$ is a non-zero integer, and $|n / 2\rangle$ denotes the supersingleton states with AdS energy $n / 2$. These solution spaces form real higher-spin representations with positive definite bilinear forms $[1,2]$. Thus, for each distinct $n$, the classical solution lends itself to be interpreted as a microstate of a Euclidean theory, rather than a Lorentzian one as is the case for the particle states; see also the Conclusions.

Looking more carefully at the classical solution for a given $n$, its Lorentz covariant field content contains an infinite tower of Weyl tensors of all spins that are spherically symmetric, static and of generalized Petrov Type D, each of which has a curvature singularity at its center; in particular, in the spin-two sector one recognizes the AdS-Schwarzschild Weyl tensor. The Weyl tensors carry asymptotic charges, that can be electric or magnetic depending on $n$, all proportional to the single deformation parameter $\nu_{n}, \mathcal{M}_{s}(\nu)=\mathcal{M}_{s}^{n} \nu_{n}$ (no sum on $n$ ). On the other hand, starting from a given Fronsdal field of a fixed Lorentz spin, the corresponding linearized Type-D sector ${ }^{5}$ is finite-dimensional, and does not lend itself to any sensible interpretation as a quantum mechanical state space. However, the interactions of the Vasiliev system create "coherent" states, consisting of all Lorentz spins, making up twisted projectors and thus facilitating a physical interpretation in terms of states in a real vector space with a Euclidean norm.

Conversely, one may consider deforming a whole tower of asymptotically defined (free) Fronsdal fields, with each spin- $s$ field carrying a charge $\mathcal{M}_{s}$. In order to use Vasiliev's theory for this purpose, one has to tune the charges so that $\mathcal{M}_{s}=\mathcal{M}_{s}(\nu)=\sum_{n} \mathcal{M}_{s}^{n} \nu_{n}$ for some ensemble $\left\{\nu_{n}\right\}[1,2]$; in particular, one may choose $\nu_{n}=\delta_{n, n_{0}} \nu_{n_{0}}$ as to activate a single microstate. In this sense, one may think of the asymptotic spin- $s$ charges $\mathcal{M}_{s}$ for $s \geqslant 3$ as "higher spin hairs" that needs to be fine-tuned to form a microstate. Moreover, it appears that the matrix $\mathcal{M}_{s}^{n}$ is non-invertible, which makes sense as the Lorentz spin is not expected to be a good observable in the strongly coupled region.

Clearly, to settle the issues of whether the singularity of the Weyl tensors is physical (not a gauge artifact), and whether or not these solutions possess an event horizon, a more detailed study of the propagation of small fluctuations over the black-hole-like solutions is required. As already mentioned, such a study is quite challenging, due to the non-locality of the interactions induced by higher spin symmetry, which requires a proper generalization of the standard geometric tools used in the case of gravity.

The aforementioned questions are tied to the issue of in which classes of functions on twistor space the master fields are allowed to take value. On general grounds, the admissible classes must form differential graded associative algebras with well-defined trace operations [92-95, 102]. We would like to stress that the class of functions making up the twistor fiber space has an impact on the boundary behaviour of the fields on the base manifold, which we expect to be dual to a set of observables, thereby determining a superselection sector of the theory; for examples, see $[18,87]$. Conversely, certain boundary

\footnotetext{
${ }^{5}$ As found in [114], for every spin $s$ it is possible to construct a solution of the spin- $s$ free Fronsdal equations as $\varphi_{\mu_{1} \ldots \mu_{s}}=\frac{M}{r} k_{\mu_{1}} \ldots k_{\mu_{s}}$, where $k_{\mu}$ is a Kerr-Schild vector. As stressed in [3], the physical higher spin gauge fields corresponding to the higher spin black hole Weyl tensors described above indeed admit such a form in a suitable gauge. It is for this reason that we refer to such fields as to the linearized Type-D sector of spin $s$.
} 
conditions in spacetime induce classes of functions in twistor fiber space whose star-product compositions must be regularized. This raises the issue of how to handle field configurations obtained by superimposing different sectors equipped with separately well-defined regular presentations. Indeed, a compatibility problem may arise upon dressing linear combinations of such linearized solutions into full solutions. This problem may have either only the trivial outcome, namely that each field configuration can only be dressed separately; or various non-trivial ones consisting of compatible combinations of regular presentations. In particular, as found in [1], the black hole solutions to the minimal bosonic model require a specific regular presentation for the projectors, which ensures associativity and traceability.

In what follows, we shall examine how to superpose particle modes over black hole modes, while we leave the issue of a proper interpretation of the latter to a future publication. Adhering to the current use in the literature, we shall therefore keep referring to them as higher spin black holes in this paper.

\subsection{Summary of our new results}

In this work, we shall extend the spherically-symmetric, static black hole solutions found in [1] by adding nonlinear time-dependent scalar field modes. To this end, we use mathematical methods that at first sight may appear to be distant from those used in ordinary treatments of gravity, but that are, on the other hand, natural from the point of view of the unfolded formulation. As mentioned above, we thus absorb the spacetime dependence into a gauge function, and construct a deformed oscillator algebra from the Weyl zero-form and twistor space connection. ${ }^{6}$ The superposition of scalar particle and black hole modes in the Weyl zero-form amounts to an expansion of its twistor fiber space dependence in terms of rotationally-invariant rank- $n$ projectors $^{7} \mathcal{P}_{n} \sim|n / 2\rangle\langle n / 2|$ and twisted projectors $\widetilde{\mathcal{P}}_{n} \sim|n / 2\rangle\langle-n / 2|$, respectively, where $|n / 2\rangle, n= \pm 1, \pm 2, \ldots$, belong to supersingleton and anti-supersingleton weight spaces for positive and negative $n$, respectively, and we have suppressed the spin degrees of freedom. These elements form an indecomposable subalgebra of the star-product algebra: evidently, the twisted projectors are not idempotent, but rather close under star products onto the projectors, which in their turn close onto themselves. Physically, this implies that, differently from black hole modes, the scalar particle modes only solve the linearized equations, and not the full equations as well. Therefore, once scalar particle modes are injected into the Weyl zero-form, the nonlinear corrections dress them into full solutions with black hole modes formed as a back-reaction.

The technical detail responsible for the aforementioned phenomenon is the presence of a Klein operator in a linear vertex in Vasiliev's equations, which has the effect of converting

\footnotetext{
${ }^{6}$ The gauge function method in four dimensions differ from that employed in three-dimensional higher spin gravity [65]. In the latter context, the analysis of the scalar propagator on higher spin black hole backgrounds is simplified by the fact that any black hole (in fact, any vacuum solution) in three spacetime dimensions is locally equivalent to $A d S_{3}$, which can be described by a vacuum gauge function. In four dimensions, however, the Weyl tensors deform the gauge function, in order for canonical Fronsdal fields to appear.

${ }^{7}$ More generally, by the Flato-Fronsdal theorem, the operators contained in $\left|j+\frac{1}{2} ;(j)\right\rangle\left\langle j^{\prime}+\frac{1}{2} ;\left(j^{\prime}\right)\right|$, with energy $j+\frac{1}{2}$ and spin $j=0, \frac{1}{2}, \ldots$, have compact spins in $(j) \otimes\left(j^{\prime}\right)$, and contribute to the harmonic expansions in terms of particle modes in fields with Lorentz spins $s=j+j^{\prime}$.
} 
particle modes into black holes modes by means of a Fourier transformation in twistor space. Schematically, the massless particle modes are first mapped, in accordance with the FlatoFronsdal theorem [20], to the aforementioned twistor-space realizations of operators $\left|\frac{n}{2}\right\rangle\left\langle\frac{n^{\prime}}{2}\right|$ $\left(n, n^{\prime}>0\right)$ in supersingleton state space [87]. These operators are then mapped via the aforementioned linear vertex to an outgoing operator of the form $\left|\frac{n}{2}\right\rangle\left\langle-\frac{n^{\prime}}{2}\right|$, where the bra is an anti-supersingleton state. At the second order, the interactions produce an admixture of supersingleton-supersingleton and supersingleton-anti-supersingleton operators, the latter corresponding to black-hole modes [1-3]. Thus, without any further fine-tuning of the initial data, the backreaction from particles produces black hole modes already at the second order of the classical perturbation theory, which one may view as a manifestation of the innate spacetime non-locality of the Vasiliev system. In the bulk of the paper, we shall fill in the details of the above sketchy derivation of this interesting backreaction mechanism.

The above results lend themselves to a holographic interpretation as follows: let us start by injecting into the bulk the states conjectured to be dual to the free vector model with conserved higher spin currents, by expanding the Weyl zero-form over operators corresponding to massless particle modes of arbitrary (integer) spin. Following these modes into the bulk, the Weyl tensors become strongly coupled, and the non-local vertices bring forth higher spin black hole modes, that blow up at the origin. In this sense, the projectors can be thought of as boundary states - since at the boundary all Weyl tensors fall off and the theory linearizes, so that one can think of preparing single Fronsdal fields - while the twisted projectors can be thought of as horizon states, as they blow up in the interior, where the fields coalesce into collective degrees of freedom that can no longer be assigned distinct Lorentz spins.

At first order, though, the black hole modes can be switched off, and it is possible to make contact with the usual perturbative scheme in Vasiliev gauge, ${ }^{8}$ as we shall see in section 5.2.2. We defer to a future study the interesting problem of whether one can, order by order, remove the Type D modes from the twistor-space connection and the spacetime gauge fields, thereby obtaining field configurations expandable over only particle modes and defining a quasi-local Fronsdal branch of moduli space, that can be a proper candidate dual of the free large- $N$ vector model.

We would also like to remark that while the internal connection does not belong to any differential graded associative algebra, the deformed oscillator variables formed from it belong to an associative subalgebra of the star product algebra for a general combined particle and black hole state. Moreover, the effect of the vacuum AdS gauge function on the deformed oscillators is to make them real analytic on the twistor base manifold, as necessary for the existence of yet another field-dependent gauge function, bringing the solution to Vasiliev's gauge, which we construct at the linearized level. We consider the fact that the solution space exists in the Vasiliev gauge as being nontrivial. This raises the prospect of using this requirement as a superselection mechanism for admissible twistor space initial data. ${ }^{9}$

\footnotetext{
${ }^{8}$ Our choice of nomenclature reflects the fact that this gauge was used by Vasiliev in his original works on the classical perturbation theory of his equations [8].

${ }^{9}$ Configurations that are singular on the twistor base manifold may instead have a role to play in a more general physical context, for example in describing nonlinear density matrices.
} 


\subsection{Plan of the paper}

In section 2, we spell out the properties of Vasiliev's equations in four dimensions that are relevant for constructing and interpreting our exact solutions. Section 3 provides the solution Ansatz in a specific holomorphic gauge. In section 4, we apply the Ansatz to the aforementioned generalized projector algebra in a specific regular presentation, and demonstrate the backreaction mechanism and the associativity of the deformed oscillator algebra in twistor space. Section 5 contains the analysis of the spacetime behaviour of the Weyl zero-form and the internal connection in the gauge reached from the holomorphic gauge by first switching on the vacuum gauge function, and then a compensating field dependent gauge function leading to the Vasiliev gauge at the linearized level; the latter gauge permits a perturbative description in terms of self-interacting Fronsdal fields (see for example $[8,9,12,13,84])$. Our conclusions and an outlook are in section 6 . The paper contains three appendices: our conventions for spinors and the gravitational background fields in anti-de Sitter spacetime are given in appendix A; useful properties of the Klein operator are contained in appendix B; finally, appendix $\mathrm{C}$ describes the method used for constructing the deformed oscillators.

\section{Vasiliev's four-dimensional bosonic models}

In this section, we describe the basic algebraic structure of Vasiliev's equations for bosonic gauge fields of all integer spins in four spacetime dimensions; in particular, the original form of Vasiliev's equations is given in eqs. (2.64)-(2.68). We would like to stress that, while our solutions only rely on the locally defined Vasiliev system, we shall here also address some global issues that may be useful in providing them with a physical interpretation, as well as in extracting Fronsdal fields from the full system. To this end, we shall introduce a related additional set of boundary conditions, including ordering prescriptions, that we propose will lead to a well-defined perturbative expansion around asymptotically anti-de Sitter spacetime in the presence of particle and black-hole modes.

\subsection{Correspondence space}

Vasiliev's higher spin gravity is formulated in terms of a finite set of master fields. Geometrically, these are horizontal differential forms on a bundle space, that we shall refer to as the correspondence space, whose fibers as well as base manifold are noncommutative. We shall denote the correspondence space, its base and its fiber space by $\mathcal{C}, \mathcal{B}$ and $\mathcal{Y}$, respectively. The fibers are symplectic, and the higher spin algebra arises naturally as symplectomorphisms, while the base can in general be a differential Poisson manifold (equipped with an integration measure). Further below, we shall largely trivialize these structures, though the requirement that higher spin gravity admits a global formulation within this category of quantum geometries may prove fruitful in constraining the theory and constructing new models.

The noncommutative structure of $\mathcal{C}$ is carried by a differential associative algebra $\Omega(\mathcal{C})$ consisting of symbols, which is a class of differential forms on $\mathcal{C}$, thought of as a classical 
manifold, equipped with a star product, differential and trace operation. Formally, one may obtain these structures by deforming the wedge product, de Rham differential and classical integration measure for smooth and bounded forms on $\mathcal{C}$ (thought of as a compact space) along a semi-classical differential Poisson structure [74-77]. For the application to higher spin gravity, however, it is crucial that these deformations are finite, as Vasiliev's theory makes explicit usage of non-trivial roots of the unity, that have no classical limit, in order to construct closed and central elements in positive form degree that can be used to build cocycles gluing sections to connections. We shall use the term master field to refer to sections and connections alike, though, more precisely, the elements in $\Omega(\mathcal{C})$ are sections, which means that the zero-forms must be bounded, while the connections are assumed to act faithfully on $\Omega(\mathcal{C})$ and have curvatures in $\Omega(\mathcal{C})$.

The choice of $\Omega(\mathcal{C})$ is dictated by the boundary conditions of the theory. We shall consider solution spaces in which the master fields can be expanded in terms of a basis of functions on $\mathcal{Y}$ star-multiplied with component forms on $\mathcal{B}$, which are determined on-shell by differential constraints. As for the section components, it is assumed that they have finite integrals over $\mathcal{B}$ in order to yield finite star products and traces; thus they should belong to $L^{1}(\mathcal{B}) .{ }^{10}$ As for connection components, it is instead assumed that they give rise to well-defined open Wilson lines, which can be implemented in noncommutative geometry using deformed oscillator algebras; for example, see [104-106].

As for the space of functions on $\mathcal{Y}$, which one may think of as the infinite-dimensional representation matrices of the theory, we shall consider modules of the underlying higher spin algebra that lend themselves to the unfolded framework for harmonic expansion on $A d S_{4}$ [87], i.e. the elements must be real-analytic in order for the Lorentz tensorial component fields to be well-defined. In the case of the Weyl zero-form, which contain the local degrees of freedom of the theory, we shall furthermore assume that its function space admits a positive definite sequi- or bilinear form in bases where compact higher spin generators are diagonalized, in order to attempt to provide the theory will a quantum-mechanical interpretation; assuming that thar these inner products are induced via the trace operation, the functions on $\mathcal{Y}$ in the Weyl zero-form must be elements in $L^{2}(\mathcal{Y})$.

Noncommutative structure. The noncommutative structure thus amounts to a differential $\widehat{d}: \Omega(\mathcal{C}) \rightarrow \Omega(\mathcal{C})$ and a compatible associative binary composition rule $(\cdot) \star(\cdot)$ : $\Omega(\mathcal{C}) \otimes \Omega(\mathcal{C}) \rightarrow \Omega(\mathcal{C})$, that are (finite) deformations of the de Rham differential and the wedge product, respectively, such that if $\widehat{f}, \widehat{g}, \widehat{h} \in \Omega(\mathcal{C})$ then ${ }^{11}$

$$
\begin{aligned}
& \widehat{d}^{2} \widehat{f}=0, \quad \widehat{d}(\widehat{f} \star \widehat{g})=(\widehat{d} \widehat{f}) \star \widehat{g}+(-1)^{\operatorname{deg}(\widehat{f})} \widehat{f} \star(\widehat{d} \widehat{g}), \\
& \widehat{f} \star(\widehat{g} \star \widehat{h})=(\widehat{f} \star \widehat{g}) \star \widehat{h} \text {. }
\end{aligned}
$$

\footnotetext{
${ }^{10}$ On $\mathbb{R}^{2}$, the integral version of the star product is equivalent to the twisting of the convolution product by a cocycle given by a phase factor, which does not affect the convergence of the integration; thus, as the convolution product closes for functions in $L^{1}\left(\mathbb{R}^{2}\right)$, so does the star product; we thank Giuseppe de Nittis for pointing out this fact to us.

${ }^{11}$ The hat on $\widehat{d}$ indicates that the it is in general a nontrivial deformation of the de Rham differential, whereas the hats on the elements in $\Omega(\mathcal{C})$ are used to distinguish them from elements on various subbundles that will be introduced below.
} 
These operations are in addition assumed to be compatible with an hermitian conjugation operation $\dagger$, viz.

$$
(\widehat{f} \star \widehat{g})^{\dagger}=(-1)^{\operatorname{deg}(\widehat{f}) \operatorname{deg}(\widehat{g})}(\widehat{g})^{\dagger} \star(\widehat{f})^{\dagger}, \quad(\widehat{d} \widehat{f})^{\dagger}=\widehat{d}\left((\widehat{f})^{\dagger}\right), \quad\left((\widehat{f})^{\dagger}\right)^{\dagger}=\widehat{f},
$$

for all $\widehat{f}, \widehat{g} \in \Omega(\mathcal{C})$. We shall assume that there exists a cover of $\mathcal{C}$ by charts and corresponding bases for local symbol calculus, which one may refer to as a quantum atlas. The corresponding similarity transformations (including transitions between charts) are given by Kontsevich gauge transformations [62], which combine bundle isomorphims (including higher spin gauge transformations) with re-orderings of symbols generated by (symmetric) polyvector fields. The resulting ambiguities are factored out at the level of classical observables given by functionals that are invariant under (small) Kontsevich gauge transformations.

Topology of correspondence space. As for the bundle structure of the correspondence space, viz.

$$
\mathcal{Y} \hookrightarrow \mathcal{C} \longrightarrow \mathcal{B}
$$

we take the fiber space $\mathcal{Y} \cong \mathbb{R}^{4}$ to be a symplectic manifold with global canonical coordinates; the structure group is thus the group of symplectomorphisms of $\mathcal{Y}$, playing the role of higher spin gauge group. ${ }^{12}$ As has already been remarked above, although $\mathcal{Y}$ is not compact, the class of functions on $\mathcal{Y}$ used to expand the sections will in effect be assumed to provide $\mathcal{Y}$ with a compact topology (at the level of the trace operation); further below, we shall achieve this by taking the functions on $\mathcal{Y}$ to be symbols of (super)traceable operators in Fock spaces (defined using Weyl order).

We furthermore assume $\mathcal{B}$ to be closed (hence compact) while allowing the (higher spin) connection to blow up on a submanifold

$$
\mathcal{S} \subset \mathcal{B},
$$

modulo gauge and ordering artifacts, that is, we assume that there exists a quantum atlas such that the connection is smooth away from $\mathcal{S}$; conversely, we assume that the singularities in the connection on $\mathcal{S}$ are not removable by going to a "finer" quantum atlas. We remark that, in this sense, large Kontsevich gauge transformations can be used to generate physical singularities in the connection whose structure define boundary states, such as, for example, conformal classes of higher spin gauge fields corresponding to sources for conserved currents on the dual conformal field theory side.

In order to describe asymptotically anti-de Sitter higher spin geometries, we shall take the differential Poisson structure on $\mathcal{C}$ to be flat and torsion free $[74,75]$, and assume a the bundle structure of $\mathcal{C}$ to be trivial with

$$
\mathcal{B} \cong \mathcal{X} \times \mathcal{Z}
$$

\footnotetext{
${ }^{12}$ The elevation of the space of horizontal forms on $\mathcal{C}$,i.e. the kernel of inner derivation along vertical vector fields, to a differential graded subalgebra of $\Omega(\mathcal{C})$ compatible with the quantum geometry, requires a differential Poisson structure with special (abelian) Killing vectors [78] along $\mathcal{Y}$ in order to project the star product and a special vertical top-form to project the quantum differential.
} 
where

$$
\mathcal{X} \cong S^{1} \times S^{3}
$$

is commutative, and

$$
\mathcal{Z} \cong S^{2} \times \bar{S}^{2}
$$

is a non-commutative space obtained by adding (commuting) points at infinity to $\mathcal{Z}^{\prime} \cong$ $\mathbb{R}^{4}[107]$. In other words, we have

$$
\mathcal{C}=\mathcal{X} \times \mathcal{T}, \quad \mathcal{T}:=\mathcal{Y} \times \mathcal{Z} \cong \mathbb{R}^{4} \times S^{2} \times \bar{S}^{2} .
$$

where $\mathcal{T}$ is thus obtained by adding points at infinity to

$$
\mathcal{T}^{\prime}:=\mathcal{Y} \times \mathcal{Z}^{\prime} \cong \mathbb{R}^{8}
$$

equipped with global canonical coordinates.

Ordering schemes and boundary conditions. In providing the (perturbatively) exact solutions, we shall start from an Ansatz in which the sections belong to the algebra

$$
\Omega(\mathcal{C})=\Omega(\mathcal{X}) \otimes \Omega(\mathcal{T}),
$$

where $\Omega(\mathcal{T})$ consists of symbols defined using Weyl order that are forms in $L^{1}(\mathcal{Z})$ valued in an extension $\mathcal{W}$ of the Weyl algebra on $\mathcal{Y}$ by inner Klein operators [107], as we shall describe further below. The trace operation on $\Omega(\mathcal{T})$ is given by integration over $\mathcal{Z}$ combined with a trace operation on $\mathcal{W}$ given by a regularized integral over $\mathcal{Y}$; see [107] for the definition. We shall then apply a large gauge transformation, and convert the sections to elements in the algebra $\Omega\left(\mathcal{T}^{\prime}\right)$ consisting of symbols defined using normal order that are real-analytic at the origin of $\mathcal{T}^{\prime}$, that is, they can be expanded in the basis of monomials in the canonical coordinates on $\mathcal{T}^{\prime}$, at generic spacetime points. As for $\mathcal{X}$, we shall keep the time-direction periodic by working with a compact spectrum of states realized in $\mathcal{W}$. Furthermore, we decompactify $S^{3}$ by allowing the connection to blow up at its south and north poles, $p_{\mathrm{S}}$ and $p_{\mathrm{N}}$, respectively, corresponding to the boundary and origin of the anti-de Sitter spacetime background, i.e. we take

$$
\mathcal{S}=S^{1} \times p_{\mathrm{S}} \times \mathcal{P}_{\mathrm{S}} \cup S^{1} \times p_{\mathrm{N}} \times \mathcal{P}_{\mathrm{N}},
$$

where $\mathcal{P}_{\mathrm{S}, \mathrm{N}}$ are planes passing through the origin of $\mathcal{T}$. We propose that requiring the connection at $p_{\mathrm{S}}$ to consist of asymptotically defined Fronsdal fields, defined order by order in perturbation theory, yields an irreducible gauge equivalence class of connections; the implementation of this boundary condition requires the aforementioned large gauge transformation, as will be discussed further below. We shall furthermore propose that the quasi-local Fronsdal branch arises upon further requiring smooth connections at $p_{\mathrm{N}}$.

As for the status of the boundary conditions on the connection, we shall implement them only to the linearized order. It remains to be seen whether they can be reached at higher orders, and, if so, whether they are non-trivial (in the sense that they select an irreducible gauge equivalence class) in view of the ambiguities residing in the form of large 
Kontsevich gauge transformations. We shall also leave for future work the issue of whether the sections in $\Omega\left(\mathcal{T}^{\prime}\right)$ can actually be mapped back to $\Omega(\mathcal{T})$ (by going from normal back to Weyl order). We remark that this would imply the Weyl zero-form is actually expandable over the basis of $\mathcal{W}$ in terms of bounded component fields, which may seem surprising in the case of the black-hole-like solutions, whose separate linearized Weyl tensors have singularities at $p_{\mathrm{N}}$. However, as we shall see, these can be summed up and converted to regular elements in the extended Weyl algebra (given by delta functions on $\mathcal{Y}$ ), at least at the linearized order. Another problem that we shall not address in any detail is whether the quasi-local Fronsdal branch can be equivalently described using an entirely normal-ordered scheme. To this end, it would be natural to equip $\Omega\left(\mathcal{T}^{\prime}\right)$ with the standard trace given by the integral over $\mathcal{T}^{\prime}$, which would require the symbols to be elements of $L^{1}\left(\mathcal{T}^{\prime}\right)$; for a brief discussion, see section 5.3.

Higher spin gauge fields and deformed oscillator algebras. Large gauge transformations are required in order to construct connections that obey the dual boundary conditions in normal and Weyl order. In normal order, the corresponding gauge condition in $\Omega\left(\mathcal{T}^{\prime}\right)$, namely, the Vasiliev gauge condition to be spelled out below, ensures that the restriction of the connection on $\mathcal{C}$ from $\mathcal{B}$ to $\mathcal{X}$, or more precisely, projecting onto the elements in $\Omega\left(\mathcal{T}^{\prime}\right)$ that are constant on $\mathcal{Z}$, defines a higher spin connection on $\mathcal{X}$ valued in a higher spin Lie algebra, to be defined below. The Vasiliev gauge condition implies that the gauging of the higher spin algebra, or unfolding procedure, yields a set of Fronsdal fields with particular self-interaction upon taking the spin-two subsector to describe a Lorentzian spacetime. Dually, for a generic point $p \in \mathcal{X}$, the restriction of the connection on $\mathcal{C}$ to $\{p\} \times \mathcal{Z}^{\prime}$ is assumed to equip $\mathcal{T}^{\prime}$ with a deformed oscillator algebra $\left.\widehat{\mathcal{A}}\right|_{p}$ that is a subalgebra of $\Omega\left(\mathcal{T}^{\prime}\right)$ and that in its turn is assumed to admit a Weyl ordered description in $\Omega(\mathcal{T})$.

We would like to stress the fact that Vasiliev's procedure is designed to describe interactions in spacetime that are dual to deformed oscillator algebras in twistor space rather than to obey any quasi-locality conditions in spacetime. Whether the resulting conversion of singularities in spacetime to distributions in twistor space provides a physically acceptable model for black holes remains to be spelled out in more detail. A promising fact, noted already in [1], is that the relevant Type D sector, which is infinite-dimensional in higher spin gravity already in the metric-like formulation, is mapped by means of a simple $\mathbb{Z}_{2}$-transformation to the ordinary massless particle spectrum consisting of lowest-weight spaces, as we shall recall below.

Local coordinates. We coordinatize $\mathcal{C}$ using $(\underline{\alpha}=(\alpha, \dot{\alpha}) ; \alpha, \dot{\alpha}=1,2)^{13}$

$$
\Xi^{\underline{M}}=\left(X^{M} ; Y^{\underline{\alpha}}\right), \quad X^{M}=\left(x^{\mu} ; Z^{\underline{\alpha}}\right), \quad\left(Y^{\underline{\alpha}} ; Z^{\underline{\alpha}}\right)=\left(y^{\alpha}, \bar{y}^{\dot{\alpha}} ; z^{\alpha},-\bar{z}^{\dot{\alpha}}\right),
$$

with reality properties

$$
\left(x^{\mu}\right)^{\dagger}=x^{\mu}, \quad\left(y^{\alpha}\right)^{\dagger}=\bar{y}^{\dot{\alpha}}, \quad\left(z^{\alpha}\right)^{\dagger}=\bar{z}^{\dot{\alpha}},
$$

\footnotetext{
${ }^{13}$ Our spinor conventions are collected in appendix A.
} 
and canonical commutation rules

$$
\left[y^{\alpha}, y^{\beta}\right]_{\star}=2 i \epsilon^{\alpha \beta}, \quad\left[z^{\alpha}, z^{\beta}\right]_{\star}=-2 i \epsilon^{\alpha \beta}, \quad\left[y^{\alpha}, z^{\beta}\right]_{\star}=0,
$$

idem their complex conjugates. We also have

$$
[\widehat{d} \Xi \underline{M}, \widehat{f}]_{\star}=0
$$

where the bracket is graded, and we choose a symbol calculus such that

$$
\widehat{d} \Xi \underline{M} \star \widehat{f}=\widehat{d} \Xi \underline{M} \wedge \widehat{f}
$$

in what follows we shall suppress the wedge product when ambiguities cannot arise. The local representatives of horizontal forms and the differential acting on them are given, respectively, by

$$
\left.\left.\widehat{f}\right|_{\text {hor }} \stackrel{\text { loc }}{=} \widehat{f}\right|_{d Y \underline{\alpha}=0}=\widehat{f}(X, d X ; Y), \quad \widehat{\left.d\right|_{\text {hor }}} \stackrel{\text { loc }}{=} \widehat{\left.d\right|_{d Y}}{ }_{d=0}=d X^{M} \partial_{M} .
$$

Star product formula. On $\mathcal{T}^{\prime}$, we shall use Vasiliev's normal ordered star product, given by the following twisted convolution formula:

$$
\begin{aligned}
& \widehat{f}_{1}(y, \bar{y}, z, \bar{z}) \star \widehat{f}_{2}(y, \bar{y}, z, \bar{z}) \\
= & \int_{\mathcal{R}_{\mathbb{R}}} \frac{d^{2} u d^{2} \bar{u} d^{2} v d^{2} \bar{v}}{(2 \pi)^{4}} e^{i\left(v^{\alpha} u_{\alpha}+\bar{v}^{\dot{\alpha}} \bar{u}_{\dot{\alpha}}\right)} \widehat{f}_{1}(y+u, \bar{y}+\bar{u} ; z+u, \bar{z}-\bar{u}) \widehat{f}_{2}(y+v, \bar{y}+\bar{v} ; z-v, \bar{z}+\bar{v}),
\end{aligned}
$$

where the integration domain

$$
\mathcal{R}_{\mathbb{R}}=\left\{\left(u_{\alpha}, \bar{u}_{\dot{\alpha}} ; v_{\alpha}, \bar{v}_{\dot{\alpha}}\right):\left(u_{\alpha}\right)^{\dagger}=u_{\alpha}, \quad\left(\bar{u}_{\dot{\alpha}}\right)^{\dagger}=\bar{u}_{\dot{\alpha}}, \quad\left(v_{\alpha}\right)^{\dagger}=v_{\alpha}, \quad\left(\bar{v}_{\dot{\alpha}}\right)^{\dagger}=\bar{v}_{\dot{\alpha}}\right\},
$$

that is, all auxiliary variables are integrated over the real line. In particular, it follows that

$$
\begin{array}{ll}
y_{\alpha} \star y_{\beta}=y_{\alpha} y_{\beta}+i \varepsilon_{\alpha \beta}, & y_{\alpha} \star z_{\beta}=y_{\alpha} z_{\beta}-i \varepsilon_{\alpha \beta}, \\
z_{\alpha} \star y_{\beta}=z_{\alpha} y_{\beta}+i \varepsilon_{\alpha \beta}, & z_{\alpha} \star z_{\beta}=z_{\alpha} z_{\beta}-i \varepsilon_{\alpha \beta},
\end{array}
$$

idem the anti-holomorphic variables. Equivalently, in terms of the creation $(+)$ and annihilation (-) operators

$$
a_{\alpha}^{ \pm}:=\frac{1}{2}\left(y_{\alpha} \pm z_{\alpha}\right)
$$

one has

$$
\begin{array}{ll}
a_{\alpha}^{-} \star a_{\beta}^{+}=a_{\alpha}^{-} a_{\beta}^{+}+i \varepsilon_{\alpha \beta}, & a_{\alpha}^{+} \star a_{\beta}^{-}=a_{\alpha}^{+} a_{\beta}^{-}, \\
a_{\alpha}^{+} \star a_{\beta}^{+}=a_{\alpha}^{+} a_{\beta}^{+}, & a_{\alpha}^{-} \star a_{\beta}^{-}=a_{\alpha}^{-} a_{\beta}^{-} .
\end{array}
$$

Thus, the star-product formula provides a realization of the operator product in terms of symbols defined in the above normal order. To be more precise, letting $\mathcal{O}_{\text {Normal }}$ denote the Wigner map that sends a classical function $\widehat{f}(y, z)$ to the operator $\mathcal{O}_{\text {Normal }}(\widehat{f}(y, z))$ with symbol $\widehat{f}(y, z)$ defined in normal order, we have

$$
\mathcal{O}_{\text {Normal }}\left(\widehat{f}_{1}(y, z) \star \widehat{f}_{2}(y, z)\right)=\mathcal{O}_{\text {Normal }}\left(\widehat{f}_{1}(y, z)\right) \mathcal{O}_{\text {Normal }}\left(\widehat{f}_{2}(y, z)\right) .
$$


Letting $\mathcal{O}_{\text {Weyl }}$ denote the corresponding map defined using Weyl order, we have

$$
\mathcal{O}_{\text {Weyl }}(f(y))=\mathcal{O}_{\text {Normal }}(f(y)), \quad \mathcal{O}_{\text {Weyl }}(f(z))=\mathcal{O}_{\text {Normal }}(f(z)) .
$$

It follows that

$$
\begin{aligned}
& \mathcal{O}_{\text {Weyl }}\left(f_{1}(y) \star f_{2}(y)\right)=\mathcal{O}_{\text {Weyl }}\left(f_{1}(y)\right) \mathcal{O}_{\text {Weyl }}\left(f_{2}(y)\right), \\
& \mathcal{O}_{\text {Weyl }}\left(f_{1}(z) \star f_{2}(z)\right)=\mathcal{O}_{\text {Weyl }}\left(f_{1}(z)\right) \mathcal{O}_{\text {Weyl }}\left(f_{2}(z)\right),
\end{aligned}
$$

and that

$$
\mathcal{O}_{\text {Normal }}\left(f_{1}(y) \star f_{2}(z)\right)=\mathcal{O}_{\text {Weyl }}\left(f_{1}(y)\right) \mathcal{O}_{\text {Weyl }}\left(f_{2}(z)\right)=\mathcal{O}_{\text {Weyl }}\left(f_{1}(y) f_{2}(z)\right),
$$

where the fact that the $Y$ and $Z$ oscillators are mutually commuting has been used in the last step. In other words, if the symbol $\widehat{f}(y, z)$ of an operator defined using normal order can be factorized as $\widehat{f}(y, z)=\sum_{\lambda} f_{\lambda}(y) \star f^{\lambda}(z)$ (over some classes of functions or distributions on $\mathcal{Y}$ and $\mathcal{Z}$ ), then its symbol defined using Weyl order is given by $\sum_{\lambda} f_{\lambda}(y) f^{\lambda}(z)$. We would like to stress, however, that, in what follows, all symbols will always be given using the normal order.

Regularization of star products. The twisted convolution formula extends the Moyal product from the space of real analytic functions to the space of Fourier transformable functions, including delta function distributions (including their derivatives). When applied to group elements and projectors, this may result in auxiliary Gaussian integrals that involve indefinite diagonalizable bilinear forms; resorting to the original Moyal-product shows that these integrals must be performed by means of analytical continuation in the eigenvalues of the bilinear forms.

\subsection{Master fields}

The connection on $\mathcal{C}$ is a one-form $\widehat{A}$ with curvature

$$
\widehat{F}:=\widehat{d} \widehat{A}+\widehat{A} \star \widehat{A},
$$

transforming in the adjoint representation of the structure group, and obeying the Bianchi identity

$$
\widehat{D} \widehat{F}:=\widehat{d} \widehat{F}+[\widehat{A}, \widehat{F}]_{\star} \equiv 0 .
$$

Higher spin gravity also makes use of twisted-adjoint [7-9], and, more generally, bi-fundamental [107] master fields, that can be introduced geometrically by replacing $\Omega(\mathcal{C})$ by $\Omega(\mathcal{C}) \times \mathcal{K} \times \mathcal{F}$, where $\mathcal{K}$ is generated by outer Klein operators $[4,7,9]$ and $\mathcal{F}$ is an internal graded Frobenius algebra [107]. Consistent truncation [107] leads to a twisted adjoint zero-form $\widehat{\Phi}$ and a pair of closed and twisted-central two-forms $(\widehat{J}, \widehat{\bar{J}})$. The resulting fields obey the reality conditions

$$
(\widehat{\Phi}, \widehat{A}, \widehat{J}, \widehat{\bar{J}})^{\dagger}=(\pi(\widehat{\Phi}),-\widehat{A},-\widehat{\bar{J}},-\widehat{J}),
$$


where $\pi$ and $\bar{\pi}$ are the involutive automorphisms defined by $\widehat{d} \pi=\pi \widehat{d}, \widehat{d} \bar{\pi}=\bar{\pi} \widehat{d}$ and

$$
\begin{array}{ll}
\pi\left(x^{\mu} ; y^{\alpha}, \bar{y}^{\dot{\alpha}} ; z^{\alpha}, \bar{z}^{\dot{\alpha}}\right)=\left(x^{\mu} ;-y^{\alpha}, \bar{y}^{\dot{\alpha}} ;-z^{\alpha}, \bar{z}^{\dot{\alpha}}\right), & \pi(\widehat{f} \star \widehat{g})=\pi(\widehat{f}) \star \pi(\widehat{g}), \\
\bar{\pi}\left(x^{\mu} ; y^{\alpha}, \bar{y}^{\dot{\alpha}} ; z^{\alpha}, \bar{z}^{\dot{\alpha}}\right)=\left(x^{\mu} ; y^{\alpha},-\bar{y}^{\dot{\alpha}} ; z^{\alpha},-\bar{z}^{\dot{\alpha}}\right), & \bar{\pi}(\widehat{f} \star \widehat{g})=\bar{\pi}(\widehat{f}) \star \bar{\pi}(\widehat{g}) .
\end{array}
$$

In order to define the twisted-adjoint representation, we introduce the graded bracket

$$
[\widehat{f}, \widehat{g}]_{\pi}:=\widehat{f} \star \widehat{g}-(-1)^{\operatorname{deg}(\widehat{f}) \operatorname{deg}(\widehat{g})} \widehat{g} \star \pi(\widehat{f}), \quad \widehat{f}, \widehat{g} \in \Omega(\mathcal{C}) .
$$

The twisted adjoint covariant derivative

$$
\widehat{D} \widehat{\Phi}:=\widehat{d} \widehat{\Phi}+[\widehat{A}, \widehat{\Phi}]_{\pi},
$$

obeys the Bianchi identity

$$
\widehat{D}^{2} \widehat{\Phi} \equiv[\widehat{F}, \widehat{\Phi}]_{\pi}
$$

The conditions on the two-form making it closed and twisted-central read

$$
\widehat{d} \widehat{J}=0, \quad[\widehat{J}, \widehat{f}]_{\pi}=0,
$$

for all $\widehat{f} \in \Omega(\mathcal{C})$. The bosonic models require the integer-spin projection

$$
\pi \bar{\pi}(\widehat{\Phi}, \widehat{A})=(\widehat{\Phi}, \widehat{A}),
$$

which together with the reality conditions lead to real Fronsdal fields with integer rank, each rank occurring once. The twisted-central elements obey the stronger conditions

$$
\pi(\widehat{J}, \widehat{\bar{J}})=\bar{\pi}(\widehat{J}, \widehat{\bar{J}})=(\widehat{J}, \widehat{\bar{J}}) .
$$

In the minimal models, the odd-spin Fronsdal fields are removed by the stronger even-spin projection

$$
\tau(\widehat{\Phi}, \widehat{A}, \widehat{J}, \widehat{\bar{J}})=(\pi(\widehat{\Phi}),-\widehat{A},-\widehat{J},-\widehat{\bar{J}}),
$$

where $\tau$ is the graded anti-automorphism defined by $\widehat{d} \tau=\tau \widehat{d}$ and

$$
\tau\left(x^{\mu} ; Y^{\underline{\alpha}} ; Z^{\underline{\alpha}}\right)=\left(x^{\mu} ; i Y^{\underline{\alpha}} ;-i Z^{\underline{\alpha}}\right), \quad \tau(\widehat{f} \star \widehat{g})=(-1)^{\widehat{f} \widehat{g}} \tau(\widehat{g}) \star \tau(\widehat{f}) ;
$$

from $\tau^{2}=\pi \bar{\pi}$ it follows that (2.42) implies (2.40).

Twisted-central and closed elements. Eq. (2.39) admits the following non-trivial solution [3]:

$$
\widehat{J}=j_{z} \star \kappa_{y}, \quad j_{z}:=-\frac{i}{4} d z^{\alpha} \wedge d z^{\beta} \varepsilon_{\alpha \beta} \kappa_{z}, \quad \kappa_{y}:=2 \pi \delta^{2}(y), \quad \kappa_{z}:=2 \pi \delta^{2}(z),
$$

where $\kappa_{y}$ is an inner Klein operator obeying

$$
\kappa_{y} \star f(y) \star \kappa_{y}=f(-y), \quad \kappa_{y} \star \kappa_{y}=1,
$$

idem $\kappa_{z}$. Thus, one may write $[3,79]$

$$
\widehat{J}=-\frac{i}{4} d z^{\alpha} \wedge d z^{\beta} \varepsilon_{\alpha \beta} \widehat{\kappa}, \quad \widehat{\kappa}:=\kappa_{y} \star \kappa_{z}=\exp \left(i y^{\alpha} z_{\alpha}\right),
$$


where thus

$$
\begin{aligned}
\widehat{\kappa} \star \widehat{f}(y, z) & =\widehat{\kappa} \widehat{f}(z, y), & \widehat{f}(y, z) \star \widehat{\kappa} & =\widehat{\kappa} \widehat{f}(-z,-y), \\
\widehat{\kappa} \star \widehat{f}(y, z) \star \kappa & =\pi(\widehat{f}(y, z)), & \widehat{\kappa} \star \widehat{\kappa} & =1 .
\end{aligned}
$$

By hermitian conjugation one obtains

$$
\widehat{\bar{J}}=-(\widehat{J})^{\dagger}=-\frac{i}{4} d \bar{z}^{\dot{\alpha}} \wedge d \dot{\bar{z}}^{\dot{\beta}} \varepsilon_{\dot{\alpha} \dot{\beta}} \widehat{\widehat{\kappa}} .
$$

\subsection{Equations of motion}

Master field equations. Vasiliev's equations of motion are given by

$$
\widehat{D} \widehat{\Phi}=0, \quad \widehat{F}+\mathcal{F}(\widehat{\Phi}) \star \widehat{J}+\overline{\mathcal{F}}(\widehat{\Phi}) \star \widehat{J}=0,
$$

where the interaction ambiguities. ${ }^{14}$

$$
\mathcal{F}=\sum_{n=0}^{\infty} f_{2 n+1}(\widehat{\Phi})(\widehat{\Phi} \star \pi(\widehat{\Phi}))^{\star n} \star \widehat{\Phi},
$$

and $\overline{\mathcal{F}}=(\mathcal{F})^{\dagger}$, where $f_{2 n+1}$ are complex-valued zero-form charges, which are functionals of $\widehat{\Phi}$ obeying

$$
\widehat{d} f_{2 n+1}=0,
$$

on-shell. Factoring out perturbatively defined redefinitions of $\widehat{\Phi}$, one has [9, 88]

$$
\begin{aligned}
& \mathcal{F}=\mathcal{B} \star \widehat{\Phi}, \quad \mathcal{B}=\exp _{\star}(i \theta(\widehat{\Phi})), \\
& \theta=\sum_{n=0}^{\infty} \theta_{2 n}(\widehat{\Phi})(\widehat{\Phi} \star \pi(\widehat{\Phi}))^{\star n},
\end{aligned}
$$

where $\theta_{2 n}$ are real-valued zero-form charges. Introducing the parity operation $P$ given by the automorphism of $\Omega(\mathcal{C})$ defined by

$$
P\left(x^{\mu} ; y^{\alpha}, \bar{y}^{\dot{\alpha}} ; z^{\alpha}, \bar{z}^{\dot{\alpha}}\right)=\left(x^{\mu} ; \bar{y}^{\dot{\alpha}}, y^{\alpha} ;-\bar{z}^{\dot{\alpha}},-z^{\alpha}\right), \quad \widehat{d} P=P \widehat{d},
$$

and by a linear action on the expansion coefficients of the master fields, it follows that

$$
P(\widehat{J}, \widehat{\bar{J}})=(\widehat{\bar{J}}, \widehat{J}) \text {. }
$$

Hence $\mathcal{B}$ breaks parity except in the following two cases [29]:

$$
\begin{aligned}
\text { Type A model (scalar) }: \theta=0, & P(\widehat{\Phi}, \widehat{A}) & =(\widehat{\Phi}, \widehat{A}), \\
\text { Type B model (pseudo-scalar) }: \theta=\frac{\pi}{2}, & P(\widehat{\Phi}, \widehat{A}) & =(-\widehat{\Phi}, \widehat{A}) .
\end{aligned}
$$

The equations of motion are Cartan integrable, and hence admit the following on-shell Cartan gauge transformations:

$$
\delta_{\widehat{\epsilon}} \widehat{\Phi}=-[\widehat{\epsilon}, \widehat{\Phi}]_{\pi}, \quad \delta_{\widehat{\epsilon}} \widehat{A}=\widehat{D} \widehat{\epsilon}:=\widehat{d} \widehat{\epsilon}+[\widehat{A}, \widehat{\epsilon}]_{\star},
$$

where the parameters obey the same kinematic conditions as $\widehat{A}$, and the two-form is treated as a background in the sense that $\delta_{\widehat{\epsilon}} \widehat{J}=0$ idem $\widehat{\bar{J}}$.

\footnotetext{
${ }^{14}$ The functionals $\mathcal{F}$ cannot be fixed by any a priori considerations on-shell. In the off-shell formulation proposed in [107], the parameters in $\mathcal{F}$ represent degrees of freedom entering via a dynamical two-form.
} 
Flat connection and deformed oscillators. In order to exhibit more explicitly the deformation of the curvature induced by the closed and twisted-central elements, we decompose

$$
\widehat{A}=\widehat{U}+\widehat{V}
$$

where

$$
\begin{aligned}
& \widehat{U}:=d x^{\mu} \widehat{U}_{\mu}(x ; Z, Y), \\
& \widehat{V}:=d Z^{\underline{\alpha}} \widehat{V}_{\underline{\alpha}}(x ; Z, Y)=d z^{\alpha} \widehat{V}_{\alpha}(x ; Z, Y)+d z^{\dot{\alpha}} \widehat{V}_{\dot{\alpha}}(x ; Z, Y),
\end{aligned}
$$

and introduce the deformed oscillators

$$
\widehat{S}_{\underline{\alpha}}:=\left(\widehat{S}_{\alpha},-\widehat{\bar{S}}_{\dot{\alpha}}\right):=Z_{\underline{\alpha}}-2 i \widehat{V}_{\underline{\alpha}}=\left(z_{\alpha}-2 i \widehat{V}_{\alpha},-\bar{z}_{\dot{\alpha}}+2 i \widehat{\bar{V}}_{\dot{\alpha}}\right) .
$$

Letting $d=d x^{\mu} \partial_{\mu}$, the master field equations can be rewritten as

$$
\begin{aligned}
d \widehat{U}+\widehat{U} \star \widehat{U} & =0, \\
d \widehat{\Phi}+\widehat{U} \star \widehat{\Phi}-\widehat{\Phi} \star \pi(\widehat{U}) & =0, \\
d \widehat{S}_{\underline{\alpha}}+\left[\widehat{U}, \widehat{S}_{\alpha}\right]_{\star} & =0, \\
\widehat{S}_{\alpha} \star \widehat{\Phi}+\widehat{\Phi} \star \pi\left(\widehat{S}_{\alpha}\right) & =0, \\
\widehat{\bar{S}}_{\dot{\alpha}} \star \widehat{\Phi}+\widehat{\Phi} \star \bar{\pi}\left(\widehat{\bar{S}}_{\dot{\alpha}}\right) & =0, \\
{\left[\widehat{S}_{\alpha}, \widehat{S}_{\beta}\right]_{\star} } & =-2 i \epsilon_{\alpha \beta}(1-\mathcal{B} \star \widehat{\Phi} \star \widehat{\kappa}), \\
{\left[\widehat{\bar{S}}_{\dot{\alpha}}, \widehat{\bar{S}}_{\dot{\beta}}\right]_{\star} } & =-2 i \epsilon_{\dot{\alpha} \dot{\beta}}(1-\overline{\mathcal{B}} \star \widehat{\Phi} \star \widehat{\bar{\kappa}}), \\
{\left[\widehat{S}_{\alpha}, \widehat{\bar{S}}_{\dot{\alpha}}\right]_{\star} } & =0 .
\end{aligned}
$$

The gauge transformations now read

$$
\delta_{\widehat{\epsilon}} \widehat{\Phi}=-[\widehat{\epsilon}, \widehat{\Phi}]_{\pi}, \quad \delta_{\widehat{\epsilon}} \widehat{S}_{\underline{\alpha}}=-\left[\widehat{\epsilon}, \widehat{V}_{\underline{\alpha}}\right]_{\star}, \quad \delta_{\widehat{\epsilon}} \widehat{U}=d \widehat{\epsilon}+[\widehat{U}, \widehat{\epsilon}]_{\star} .
$$

Thus, the master field equations describe a flat connection on $\mathcal{X}$ and a covariantly constant deformed oscillator algebra

$$
\widehat{\mathcal{A}}:=\left\{(\widehat{\Phi} \star \widehat{\kappa})^{\star k} \star(\Phi \star \widehat{\widehat{\kappa}})^{\star \bar{k}} \star \widehat{S}_{\left(\underline{\alpha}_{1}\right.} \star \cdots \star \widehat{S}_{\left.\underline{\alpha}_{m}\right)}\right\},
$$

generated by the internal master fields $\left(\widehat{\Phi}, \widehat{S}_{\underline{\alpha}}\right)$. These two objects belong to subalgebras of $\Omega\left(\mathcal{T}^{\prime}\right)$ that we expect to be determined by the boundary conditions, as we shall examine in more detail in the case of higher spin black holes and fluctuation fields.

Lorentz covariantization. To obtain a manifestly locally Lorentz covariant formulation one introduces a canonical Lorentz connection $\left(\omega^{\alpha \beta}, \bar{\omega}^{\dot{\alpha} \dot{\beta}}\right)$ by means of the field redefinition $[9,84,88]$

$$
\widehat{W}:=\widehat{U}-\widehat{K}, \quad \widehat{K}:=\frac{1}{4 i}\left(\omega^{\alpha \beta} \widehat{M}_{\alpha \beta}+\bar{\omega}^{\dot{\alpha} \dot{\beta}} \widehat{\bar{M}}_{\dot{\alpha} \dot{\beta}}\right)
$$

where

$$
\widehat{M}_{\alpha \beta}:=\widehat{M}_{\alpha \beta}^{(0)}+\widehat{M}_{\alpha \beta}^{(S)}, \quad \widehat{M}_{\dot{\alpha} \dot{\beta}}=\widehat{M}_{\dot{\alpha} \dot{\beta}}^{(0)}+\widehat{M}_{\dot{\alpha} \dot{\beta}}^{(\bar{S})}
$$


are the full Lorentz generators, consisting of the internal part

$$
\widehat{M}_{\alpha \beta}^{(0)}:=y_{(\alpha} \star y_{\beta)}-z_{(\alpha} \star z_{\beta)}, \quad \widehat{M}_{\dot{\alpha} \dot{\beta}}^{(0)}:=\bar{y}_{(\dot{\alpha}} \star \bar{y}_{\dot{\beta})}-\bar{z}_{(\dot{\alpha}} \star \bar{z}_{\dot{\beta})},
$$

rotating the $Y$ and $Z$ oscillators, and the external part

$$
\widehat{M}_{\alpha \beta}^{(S)}:=\widehat{S}_{(\alpha} \star \widehat{S}_{\beta)}, \quad \widehat{\bar{M}}_{\dot{\alpha} \dot{\beta}}^{(\bar{S})}:=\widehat{\bar{S}}_{(\dot{\alpha}} \star \widehat{\bar{S}}_{\dot{\beta})},
$$

rotating the spinor indices carried by $\left(\widehat{S}_{\alpha}, \widehat{\bar{S}}_{\dot{\alpha}}\right)$. As a result, the master equations read

$$
\begin{aligned}
\nabla \widehat{W}+\widehat{W} \star \widehat{W}+\frac{1}{4 i}\left(r^{\alpha \beta} \widehat{M}_{\alpha \beta}+\bar{r}^{\dot{\alpha} \dot{\beta}} \widehat{\bar{M}}_{\dot{\alpha} \dot{\beta}}\right) & =0, \\
\nabla \widehat{\Phi}+\widehat{W} \star \widehat{\Phi}-\widehat{\Phi} \star \pi(\widehat{W}) & =0, \\
\nabla \widehat{S}_{\alpha}+\widehat{W} \star \widehat{S}_{\alpha}-\widehat{S}_{\alpha} \star \widehat{W} & =0, \\
\nabla \widehat{\bar{S}}_{\dot{\alpha}}+\widehat{W} \star \widehat{\bar{S}}_{\dot{\alpha}}-\widehat{\bar{S}}_{\dot{\alpha}} \star \widehat{W} & =0, \\
\widehat{S}_{\alpha} \star \widehat{\Phi}+\widehat{\Phi} \star \pi\left(\widehat{S}_{\alpha}\right) & =0, \\
\widehat{\bar{S}}_{\dot{\alpha}} \star \widehat{\Phi}+\widehat{\Phi} \star \widehat{\pi}_{(}\left(\widehat{\bar{S}}_{\dot{\alpha}}\right) & =0, \\
{\left[\widehat{S}_{\alpha}, \widehat{S}_{\beta}\right]_{\star} } & =-2 i \epsilon_{\alpha \beta}(1-\mathcal{B} \star \widehat{\Phi} \star \widehat{\kappa}), \\
{\left[\widehat{\bar{S}}_{\dot{\alpha}}, \widehat{\bar{S}}_{\dot{\beta}}\right]_{\star} } & =-2 i \epsilon_{\dot{\alpha} \dot{\beta}}(1-\overline{\mathcal{B}} \star \widehat{\Phi} \star \widehat{\kappa}), \\
{\left[\widehat{S}_{\alpha}, \widehat{\bar{S}}_{\dot{\alpha}}\right]_{\star} } & =0,
\end{aligned}
$$

where $r^{\alpha \beta}:=d \omega^{\alpha \beta}+\omega^{\alpha \gamma} \omega^{\beta}$ and $\bar{r}^{\dot{\alpha} \dot{\beta}}:=d \bar{\omega}^{\dot{\alpha} \dot{\beta}}+\omega^{\dot{\alpha} \dot{\gamma}} \omega^{\dot{\beta}} \dot{\gamma}$, and

$$
\begin{aligned}
& \nabla \widehat{W}:=d \widehat{W}+\frac{1}{4 i}\left[\omega^{\alpha \beta} \widehat{M}_{\alpha \beta}^{(0)}+\bar{\omega}^{\dot{\alpha} \dot{\beta}} \widehat{\bar{M}}_{\dot{\alpha} \dot{\beta}}^{(0)}, \widehat{W}\right]_{\star}, \\
& \nabla \widehat{\Phi}:=d \widehat{\Phi}+\frac{1}{4 i}\left[\omega^{\alpha \beta} \widehat{M}_{\alpha \beta}^{(0)}+\bar{\omega}^{\dot{\alpha} \dot{\beta}} \widehat{\bar{M}}_{\dot{\alpha} \dot{\beta}}^{(0)}, \widehat{\Phi}\right]_{\star}, \\
& \nabla \widehat{S}_{\alpha}:=d \widehat{S}_{\alpha}+\omega_{\alpha}^{\beta} \widehat{S}_{\beta}+\frac{1}{4 i}\left[\omega^{\beta \gamma} \widehat{M}_{\beta \gamma}^{(0)}+\bar{\omega}^{\dot{\beta} \dot{\gamma}} \widehat{M}_{\dot{\beta} \dot{\gamma}}^{(0)}, \widehat{S}_{\alpha}\right]_{\star}, \\
& \nabla \widehat{S}_{\dot{\alpha}}:=d \widehat{S}_{\dot{\alpha}}+\bar{\omega}_{\dot{\alpha}} \dot{\beta} \widehat{\bar{S}}_{\dot{\beta}}+\frac{1}{4 i}\left[\omega^{\beta \gamma} \widehat{M}_{\beta \gamma}^{(0)}+\bar{\omega}^{\dot{\beta} \dot{\gamma}} \widehat{M}_{\dot{\beta} \dot{\gamma}}^{(0)}, \widehat{\bar{S}}_{\dot{\alpha}}\right]_{\star} .
\end{aligned}
$$

The field redefinition implies a local shift-symmetry with parameter $\left(\varsigma^{\alpha \beta}, \bar{\varsigma}^{\dot{\alpha} \dot{\beta}}\right)=$ $d X^{M}\left(\varsigma_{M}^{\alpha \beta}, \bar{\varsigma}_{M}^{\dot{\alpha} \dot{\beta}}\right)$ defined by

$$
\delta_{\varsigma}\left(\widehat{U}, \widehat{\Phi}, \widehat{S}_{\alpha}, \widehat{\bar{S}}_{\dot{\alpha}}\right)=0, \quad \delta_{\varsigma}\left(\omega^{\alpha \beta}, \bar{\omega}^{\dot{\alpha} \dot{\beta}}\right)=\left(\varsigma^{\alpha \beta}, \bar{\varsigma}^{\dot{\alpha} \dot{\beta}}\right) \Rightarrow \delta_{\varsigma} \widehat{W}=-\frac{1}{4 i}\left(\varsigma^{\alpha \beta} \widehat{M}_{\alpha \beta}+\bar{\varsigma}^{\dot{\alpha} \dot{\beta}} \widehat{\bar{M}}_{\dot{\alpha} \dot{\beta}}\right),
$$

which can be used to embed the canonical Lorentz connection into the full master fields by imposing

$$
\left.\frac{\partial^{2}}{\partial y^{\alpha} \partial y^{\beta}} \widehat{W}\right|_{Y=Z=0}=0,\left.\quad \frac{\partial^{2}}{\partial \bar{y}^{\dot{\alpha}} \partial \bar{y}^{\dot{\beta}}} \widehat{W}\right|_{Y=Z=0}=0 .
$$


Perturbatively defined Fronsdal fields. To obtain a perturbative expansion in terms of self-interacting Fronsdal fields that is locally Lorentz covariant and diffeomorphism invariant on $\mathcal{X}$, one proceeds by imposing the initial conditions

$$
\left.\widehat{\Phi}\right|_{Z=0}=\Phi,\left.\quad \widehat{W}\right|_{Z=0}=W,
$$

where $W$ is valued in the bosonic higher spin algebra $\mathfrak{h s}_{1}(4)$ or in its minimal projection $\mathfrak{h} \mathfrak{s}(4)$ and $\Phi$ in the corresponding twisted-adjoint representation [4, 7-9, 84], and the Vasiliev gauge condition

$$
z^{\alpha} \widehat{V}_{\alpha}+\bar{z}^{\dot{\alpha}} \widehat{\bar{V}}_{\dot{\alpha}}=0
$$

in normal order, after which it is possible to solve the constraints involving $Z$-derivatives in a perturbative expansion in terms of $\Phi$ while maintaining real-analyticity on $\mathcal{T}$. The remaining constraints can then be shown to hold on $\mathcal{X} \times \mathcal{Z}$ provided they hold at $\mathcal{X} \times$ $\{Z=0\}$, where they define a non-linear free differential algebra on $\mathcal{X}$ generated by $\Phi$ and $W[4,8,84] .{ }^{15}$ Assuming furthermore that the Vasiliev frame field

$$
e_{\alpha \dot{\alpha}}:=\left.\frac{\partial^{2}}{\partial y^{\alpha} \partial \bar{y}^{\dot{\alpha}}} W\right|_{Y=0}
$$

is invertible, the free differential algebra yields i) algebraic constraints that express $(\Phi, W)$ in terms of a remaining set of dynamical fields, namely $e^{\alpha \dot{\alpha}}$, the scalar ${ }^{16}$

$$
\phi:=\left.\Phi\right|_{Y=0}
$$

and the Fronsdal tensors $(s \geqslant 1, s \neq 2)$

$$
\phi_{\mu(s)}:=\left.2 i e_{\left(\mu_{1}\right.}^{\alpha_{1} \dot{\alpha}_{1}} \cdots e_{\mu_{s-1}}^{\alpha_{s-1} \dot{\alpha}_{s-1}} \frac{\partial^{2 s-2}}{\partial y^{\alpha_{1}} \cdots \partial y^{\alpha_{s-1}} \bar{\partial} \bar{y}^{\dot{\alpha}_{1}} \cdots \bar{\partial} \bar{y}^{\dot{\alpha}_{s-1}}} W_{\left.\mu_{s}\right)}\right|_{Y=0},
$$

modulo auxiliary gauge symmetries; ii) dynamical metric-like equations of motion; and iii) Bianchi identities; for details, see appendix D in [1].

As for the auxiliary fields, of particular interest are the pure Weyl tensors $C_{\alpha(2 s)}(s \geqslant 1)$ that make up the generating function $(s \geqslant 0)$

$$
C:=\left.\Phi\right|_{\bar{y}=0}, \quad C_{\alpha(2 s)}:=\left.\frac{\partial^{2 s}}{\partial y^{\alpha_{1}} \cdots \partial y^{\alpha_{2 s}}} C\right|_{y=0} .
$$

The remaining components of $\Phi$ are given by all possible derivatives of the Weyl tensors that are non-vanishing on-shell.

\footnotetext{
${ }^{15}$ Concerning the Vasiliev gauge condition (2.87), prior to Lorentz covariantization, it ensures that terms involving $\partial_{\mu} \Phi$ drop out from the perturbative expression for $\widehat{A}_{\mu}$; for details, see [83]. Thus, a modification of (2.87) to $z^{\alpha} \widehat{V}_{\alpha}+\bar{z}^{\dot{\alpha}} \widehat{\bar{V}}_{\dot{\alpha}}=\widehat{\Upsilon}$, where $\widehat{\Upsilon}$ is a Lorentz singlet with a perturbative expansion starting at the second order, leads to non-canonical higher order corrections to the Cartan curvature of $W$ of involving $\widehat{\Upsilon}$ and its twistor space derivatives at $Z=0$. Eliminating these terms perturbatively, using the constraint on $\Phi$, yields a canonical Cartan curvature for $W$.

${ }^{16}$ Under the parity operation $P$, one has $P(\phi)=\phi$ in the type A model, and $P(\phi)=-\phi$ in the type B model.
} 
The following remarks are in order:

The Vasiliev gauge is required at the linearized level in order to obtain a Lorentz covariant description of free Fronsdal fields on the mass shell. Beyond this order, we observe that: i) eq. (2.87) is manifestly Lorentz covariant and conveniently removes all gauge artifacts up to residual $\mathfrak{h s}_{1}(4)$ gauge transformations on $\mathcal{X}$; ii) It also yields perturbatively well-defined master fields in regular sub-classes of $\Omega\left(\mathcal{T}^{\prime}\right)$ for generic points in $\mathcal{X}$ as introduced in [7, 64] and further developed in [94]; iii) Provided that the asymptotic spacetime gauge fields defined in normal order are Fronsdal fields order-by-order in classical perturbation theory, and that the master fields can be mapped to $\Omega(\mathcal{T})$, which ensures finite invariants given by integrals $\mathcal{B}$ and traces over $\mathcal{W}$, then any modification of (2.87) preserving the topology of $\Omega(\mathcal{T})$ will not affect the invariants, and hence lead to a physically equivalent description of higher spin dynamics. In what follows, however, we shall impose the Vasiliev gauge only at the leading order pending further consolidation of the above approach.

Secondly, Vasiliev's procedure yields Fronsdal field interactions in the Vasiliev gauge that are highly non-local in the sense that the field redefinition [95] mapping them to the quasi-local Fronsdal theory [109-111] is large in the metric-like sense [98]. Moreover, as found in [92, 93], this procedure yields classical fields that do not lend themselves to on-shell amplitude computations using the prescription of [60,61], which assumes the existence of a quasi-local action principle with canonical self-adjoint metric-like kinetic terms in antide Sitter spacetime. In response to these subtleties, criteria for classifying the locality properties of interactions, field redefinitions and gauge transformations given in twistor space have been proposed in [94], though it remains to adapt them to the non-polynomial classes of initial data describing black holes and massless particles, and to furthermore verify the proposal at the level of the prescription of $[60,61]$. The appropriate interpretation of the quasi-local Fronsdal theory may thus be as a quantum effective theory [99].

As for the underlying microscopic action for Vasiliev's full equations, an alternative proposal formulated directly in terms of the master fields in correspondence space, has been given in $[15,107]$, whereby the amplitudes are to be obtained by evaluating the on-shell action on classical master fields (containing asymptotically anti-de Sitter regions) obtained by dressing free Fronsdal fields expanded over suitable higher spin representations. Thus, the findings of the present paper may serve as a starting point for corresponding amplitude computations, once an appropriate on-shell action has been found, a problem which is currently under investigation [15, 102, 107].

\section{Solution method}

In this section, we give the method based on gauge functions and separation of variables in twistor space that we shall use to solve Vasiliev's equations. In particular, by going to a convenient gauge, we shall provide a perturbatively defined solution for general zero-form initial data.

\subsection{Gauge functions}

The basic idea is to build families of exact solutions in gauges in which the spacetime connection vanishes. The latter can then be switched on and the solutions brought to the 
Vasiliev gauge by means of large gauge transformations. To this end, one makes use of the fact that the commutative nature of $\mathcal{X}$ implies that, locally on $\mathcal{X}$, the general solution to eqs. (2.64) and (2.65) is given by

$$
\widehat{U}=\widehat{g}^{-1} \star d \widehat{g}, \quad \widehat{\Phi}=\widehat{g}^{-1} \star \widehat{\Phi}^{\prime} \star \pi(\widehat{g}), \quad \widehat{S}_{\underline{\alpha}}=\widehat{g}^{-1} \star \widehat{S}_{\underline{\alpha}}^{\prime} \star \widehat{g},
$$

where $\widehat{g}(x, Y, Z)$ is a gauge function and

$$
\partial_{\mu} \widehat{\Phi}^{\prime}=\partial_{\mu} \widehat{S}_{\underline{\alpha}}^{\prime}=0 .
$$

By a choice of coordinates, we may assume that

$$
\left.\widehat{g}\right|_{x=0}=1, \quad\left(\widehat{\Phi}^{\prime}, \widehat{S}_{\underline{\alpha}}^{\prime}\right)=\left.\left(\widehat{\Phi}, \widehat{S}_{\underline{\alpha}}\right)\right|_{x=0} .
$$

The remaining master field equations read

$$
\begin{aligned}
\widehat{S}_{\alpha}^{\prime} \star \widehat{\Phi}^{\prime}+\widehat{\Phi}^{\prime} \star \pi\left(\widehat{S}_{\alpha}^{\prime}\right) & =0, \\
\widehat{\bar{S}}_{\dot{\alpha}}^{\prime} \star \widehat{\Phi}^{\prime}+\widehat{\Phi}^{\prime} \star \bar{\pi}\left(\widehat{\bar{S}}_{\dot{\alpha}}^{\prime}\right) & =0 \\
{\left[\widehat{S}_{\alpha}^{\prime}, \widehat{S}_{\beta}^{\prime}\right]_{\star} } & =-2 i \epsilon_{\alpha \beta}\left(1-\mathcal{B}^{\prime} \star \widehat{\Phi}^{\prime} \star \kappa\right), \\
{\left[\widehat{\bar{S}}_{\dot{\alpha}}^{\prime}, \widehat{\bar{S}}_{\dot{\beta}}^{\prime}\right]_{\star} } & =-2 i \epsilon_{\dot{\alpha} \dot{\beta}}\left(1-\overline{\mathcal{B}}^{\prime} \star \widehat{\Phi}^{\prime} \star \bar{\kappa}\right) \\
{\left[\widehat{S}_{\alpha}^{\prime}, \widehat{\bar{S}}_{\dot{\alpha}}^{\prime}\right]_{\star} } & =0,
\end{aligned}
$$

to be solved subject to boundary conditions on $\left(\widehat{\Phi}^{\prime}, \widehat{S}_{\underline{\alpha}}^{\prime}\right)$ on $\mathcal{T}$. We shall focus on classical solutions that admit perturbative expansions

$$
\widehat{\Phi}^{\prime}=\sum_{n=1}^{\infty} \widehat{\Phi}^{\prime(n)}, \quad \widehat{S}_{\underline{\alpha}}^{\prime}=\sum_{n=0}^{\infty} \widehat{S}_{\underline{\alpha}}^{\prime(n)} \equiv Z_{\underline{\alpha}}-2 i \sum_{n=0}^{\infty} \widehat{V}_{\underline{\alpha}}^{\prime(n)},
$$

where $\left(\widehat{S}_{\underline{\alpha}}^{\prime(n)}, \widehat{\Phi}^{\prime(n)}\right)$ is an $n$-linear functional in the integration constant

$$
\Phi^{\prime}(Y)=\left.\widehat{\Phi}^{\prime}(Y, Z)\right|_{Z=0},
$$

and $\widehat{S}_{\underline{\alpha}}^{(0)}$ is a flat connection obeying

$$
\left[\widehat{S}_{\underline{\alpha}}^{\prime(0)}, \widehat{S}_{\underline{\beta}}^{(0)}\right]_{\star}=-2 i C_{\underline{\alpha \beta}}:=-2 i\left(\begin{array}{cc}
\epsilon_{\alpha \beta} & 0 \\
0 & \epsilon_{\dot{\alpha} \dot{\beta}}
\end{array}\right),
$$

which admit non-trivial solutions obtained by activating Fock space projectors on $\mathcal{Y} \times \mathcal{Z}$; see appendix $\mathrm{C}$ for details.

In order to obtain solutions containing asymptotically anti-de Sitter regions with free Fronsdal (gauge) fields, we use a gauge function $\widehat{G}$ subject to the Vasiliev gauge condition, viz.

$$
Z^{\underline{\alpha}}\left((\widehat{G})^{-1} \star \widehat{S}_{\underline{\alpha}}^{\prime} \star \widehat{G}\right)=0,
$$

and a vacuum condition

$$
\left.\widehat{G}\right|_{\Phi^{\prime}=0}=L
$$


where $L: \mathcal{X} \rightarrow \exp \mathfrak{h}_{1}(4)$; for the sake of simplicity, we shall choose

$$
L: \mathcal{X} \rightarrow \mathrm{SO}(2,3) / \mathrm{SO}(1,3)
$$

such that $L^{-1} \star d L$ describes anti-de Sitter spacetime.

We recall from section 3.2 that eq. (3.10) is necessary at the linearized level but optional at higher orders, though under extra assumptions on the topology of $\mathcal{Z}$ and the nature of the physical observables of the theory, one may argue that it can be imposed to all orders. We would also like to stress that in order for $\widehat{G}$ to be of physical relevance it has to be a large gauge tranformation, which affects the asymptotics of the fields on $\mathcal{X} \times \mathcal{Z}$, and some observables of the theory. The space of such transformations consists of equivalence classes formed by factoring out the proper (or small) gauge transformations, which by their definition do not affect any observable, but that may nonetheless be useful in order to remove physically irrelevant coordinate singularities and other inconvenient gauge artifacts.

Having obtained $\widehat{G}$ (as a functional of $L$ and $\Phi^{\prime}$ ), the generating functions $C$ and $W$ of the pure Weyl tensors, including the physical scalar field, and of the Fronsdal gauge fields, including the spin-two frame field, defined in eqs. (2.91) and (2.86), respectively, are given by

$$
\begin{aligned}
C & =\left.\left(\widehat{G}^{-1} \star \widehat{\Phi}^{\prime} \star \pi(\widehat{G})\right)\right|_{Z=0, \bar{y}=0}, \\
W & =\left.\left[\widehat{G}^{-1} \star d \widehat{G}-\frac{1}{4 i}\left(\omega^{\alpha \beta}\left(y_{\alpha} \star y_{\beta}+\widehat{G}^{-1} \star \widehat{S}_{\alpha}^{\prime} \star \widehat{S}_{\beta}^{\prime} \star \widehat{G}\right)+\text { h.c. }\right)\right]\right|_{Z=0} ;
\end{aligned}
$$

finally, the condition (2.85) is imposed as to determine $\left(\omega_{\mu}^{\alpha \beta}, \bar{\omega}_{\mu}^{\dot{\alpha} \dot{\beta}}\right)$.

We remark that at the classical level, the fields $\left(\widehat{\Phi}^{\prime}, \widehat{S}_{\alpha}^{\prime}\right)$ and the gauge function, which must belong to an associative algebra, may be singular or given by distributions on $\mathcal{T}$, as long as $\left(\widehat{W}, \widehat{\Phi}, \widehat{S}_{\alpha}\right)$ are real-analytic on $\mathcal{T}$ for generic points in $\mathcal{X}$. At the semi-classical level, stronger conditions arise from demanding that $\left(\widehat{W}, \widehat{\Phi}, \widehat{S}_{\underline{\alpha}}\right)$ belong to an associative bundle over $\mathcal{X}$ with well-defined invariants, whose construction is beyond the scope of this work.

In summary so far, the gauge function method gives rise to solution spaces that depend on the following classical moduli (see also [1] and references therein):

(i) the constants entering via the parametrization of $\Phi^{\prime}(Y)$, which describe local degrees of freedom;

(ii) the degrees of freedom entering via the homogenous solutions to the Vasiliev gauge condition modulo proper gauge transformations, that is, the space of boundary gauge functions $\left.\left.\widehat{G}\right|_{\partial \mathcal{X} \times\{Z=0\}} \sim L\right|_{\partial \mathcal{X}}$

(iii) star-product projectors entering via the flat connections $\widehat{V}^{\prime(0)}$ on $\mathcal{Z}$;

(iv) windings contained in the transition functions entering via the atlas for $\mathcal{X}$.

In what follows, we shall activate (i) and (iii). 


\subsection{Separation of variables in twistor space and holomorphicity}

The deformed oscillator problem (3.4)-(3.6) can be solved formally by factorizing the dependencies on $Y$ and $Z$, and assuming that the internal connection $\widehat{V}^{\prime}$ is the sum of a holomorphic and an anti-holomorphic one-form on $\mathcal{Z}$ that can be expanded perturbatively in the zero-form initial data $\Phi^{\prime}$ defined in (3.15). One can show that it is in addition possible to assume that the zero-form is uncorrected, leading to an Ansatz of the form

$$
\begin{aligned}
\widehat{\Phi}^{\prime}(Y, Z) & =\Phi^{\prime}(Y) \equiv \Psi(Y) \star \kappa_{y} \equiv \bar{\Psi}(Y) \star \bar{\kappa}_{\bar{y}}, \\
\widehat{V}_{\alpha}^{\prime}(Y, Z) & =\sum_{n=0}^{\infty}(\Psi(Y))^{\star n} \star V_{\alpha}^{(n)}(z), \\
\widehat{V}_{\dot{\alpha}}^{\prime}(Y, Z) & =\sum_{n=0}^{\infty}(\bar{\Psi}(Y))^{\star n} \star \bar{V}_{\dot{\alpha}}^{(n)}(\bar{z})
\end{aligned}
$$

where $\kappa_{y}$ and $\bar{\kappa}_{\bar{y}}$ are defined in (2.44), and we have implicitly defined

$$
\Psi:=\Phi^{\prime} \star \kappa_{y}, \quad \bar{\Psi}:=\Phi^{\prime} \star \bar{\kappa}_{\bar{y}} .
$$

The reality conditions imply that

$$
\bar{\Psi}=\Psi^{\dagger}, \quad\left(V_{\alpha}^{(n)}(z)\right)^{\dagger}=-\bar{V}_{\dot{\alpha}}^{(n)}(\bar{z}),
$$

while the bosonic projection gives

$$
\pi \bar{\pi}(\Psi)=\Psi, \quad[\Psi, \bar{\Psi}]_{\star}=0 .
$$

As for the internal connection, the bosonic projection combined with the holomorphicity implies that

$$
\pi\left(V_{\alpha}^{(n)}\right)=-V_{\alpha}^{(n)}, \quad \bar{\pi}\left(\bar{V}_{\dot{\alpha}}^{(n)}\right)=-\bar{V}_{\dot{\alpha}}^{(n)} .
$$

We shall refer to the above Ansatz as the holomorphic gauge, noting the residual symmetry under gauge transformations with holomorphically factorized gauge functions

$$
\widehat{N}^{\prime}=\widehat{h}^{\prime}(\Psi, z) \star \widehat{\bar{h}}^{\prime}(\bar{\Psi}, \bar{z}), \quad \pi\left(\widehat{h}^{\prime}\right)=\widehat{h}^{\prime}, \quad \bar{\pi}\left(\hat{\bar{h}}^{\prime}\right)=\widehat{\bar{h}}^{\prime}, \quad\left(\widehat{h}^{\prime}\right)^{\dagger}=\widehat{\bar{h}}^{\prime} .
$$

In this gauge, one has $\widehat{F}_{\alpha \dot{\alpha}}=0$ and $\widehat{D}_{\alpha}^{\prime} \widehat{\Phi}^{\prime}=0$, idem for the hermitian conjugate; the former follows immediately from holomorphicity and (3.20), while the latter can be seen by rewriting

$$
\widehat{D}_{\alpha}^{\prime} \widehat{\Phi}^{\prime}=\sum_{n=0}^{\infty} \Psi^{\star n+1} \star\left\{V_{\alpha}^{(n)}, \kappa_{z}\right\}_{\star}=0,
$$

and using (3.21). The remaining constraints on $\widehat{F}_{\alpha \beta}^{\prime}$ and $\widehat{\bar{F}}_{\dot{\alpha} \dot{\beta}}^{\prime}$ (see eqs. (3.5)) now read

$$
\begin{array}{r}
\partial_{[\alpha} \widehat{V}_{\beta]}^{\prime}+\widehat{V}_{[\alpha}^{\prime} \star \widehat{V}_{\beta]}^{\prime}=-\frac{i}{4} \epsilon_{\alpha \beta} \mathcal{B}(\Psi \star \Psi) \star \Psi \star \kappa_{z}, \\
\partial_{[\dot{\alpha}} \widehat{\bar{V}}_{\dot{\beta}]}^{\prime}+\widehat{\bar{V}}_{[\dot{\alpha}}^{\prime} \star \widehat{\bar{V}}_{\dot{\beta}]}^{\prime}=-\frac{i}{4} \epsilon_{\dot{\alpha} \dot{\beta}} \overline{\mathcal{B}}(\bar{\Psi} \star \bar{\Psi}) \star \bar{\Psi} \star \bar{\kappa}_{\bar{z}},
\end{array}
$$


which are formally equivalent to those solved in [1] using the extension of the non-perturbative method of [64] given in $[1,66]$ as to include non-trivial flat connections $\widehat{V}_{\underline{\alpha}}^{\prime(0)}$; for details, see appendix C. For the sake of simplicity, we shall henceforth take

$$
\widehat{S}_{\underline{\alpha}}^{\prime(0)}=Z_{\underline{\alpha}}, \quad \mathcal{B}=b:=e^{i \theta_{0}},
$$

stressing that there is no technical obstruction to generalize the results as to include the full interaction ambiguity and non-trivial vacuum connections on $\mathcal{Z}$.

Next, we shall choose a particular solution for the internal connection. To this end, we use a spin-frame $u_{\alpha}^{ \pm}$obeying

$$
u^{\alpha+} u_{\alpha}^{-}=1
$$

to define conjugate variables

$$
z^{ \pm}:=u^{ \pm \alpha} z_{\alpha}, \quad\left[z^{-}, z^{+}\right]_{\star}=-2 i,
$$

that allows us to represent $\kappa_{z}$, appearing in the right-hand side of (3.5), as the following delta function sequence [1]: ${ }^{17}$

$$
\kappa_{z}=\lim _{\epsilon \rightarrow 0^{+}} \frac{1}{\epsilon} e^{-\frac{i}{\epsilon} w_{z}}, \quad w_{z}:=z^{+} z^{-}=\frac{1}{2} z^{\alpha} z^{\beta} \mathcal{D}_{\alpha \beta}, \quad \mathcal{D}_{\alpha \beta}:=2 u_{(\alpha}^{+} u_{\beta)}^{-} .
$$

Fixing the residual holomorphic gauge symmetries, we can construct the particular solution

$$
\widehat{V}_{\alpha}^{\prime}=2 i \sum_{n=1}^{\infty}\left(\begin{array}{c}
1 / 2 \\
n
\end{array}\right)\left(-\frac{b}{2}\right)^{n} \int_{-1}^{1} \frac{d t}{(t+1)^{2}} \frac{\left(\log \left(1 / t^{2}\right)\right)^{n-1}}{(n-1) !} z_{\alpha} e^{i \frac{t-1}{t+1} w_{z}} \star \Psi^{\star n} .
$$

Indeed, expansion of eqs. (3.24)-(3.25) in $\star$-powers of $\Psi$ yields

$$
\begin{gathered}
\partial_{[\alpha} V_{\beta]}^{(1)}+\frac{i}{4} \epsilon_{\alpha \beta} b \kappa_{z}=0, \\
\partial_{[\alpha} V_{\beta]}^{(n)}+\frac{1}{2} \sum_{p+q=n}\left[V_{\alpha}^{(p)}, V_{\beta}^{(q)}\right]_{\star}=0, \quad n \geq 2,
\end{gathered}
$$

which are solved by the coefficient of $\Psi^{\star n}$ in (3.30), i.e.

$$
V_{\alpha}^{(n)}=2 i\left(\begin{array}{c}
1 / 2 \\
n
\end{array}\right)\left(-\frac{b}{2}\right)^{n} \int_{-1}^{1} \frac{d t}{(t+1)^{2}} \frac{\left(\log \left(1 / t^{2}\right)\right)^{n-1}}{(n-1) !} z_{\alpha} e^{i \frac{t-1}{t+1} w_{z}} .
$$

The above particular solution obeys $z^{\alpha} V_{\alpha}^{(n)}=0$, and is given by a distribution that is not real-analytic at $z^{\alpha}=0$; thus, the symbol of $\widehat{V}_{\alpha}^{\prime}$ in Weyl order has the same properties.

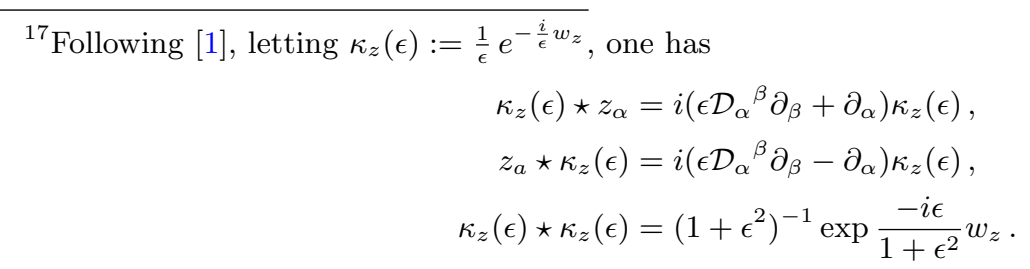

Assuming that $\check{\kappa}_{z}:=\lim _{\epsilon \rightarrow 0^{+}} \kappa_{z}(\epsilon)$ exists as a distribution, it follows that

$$
\check{\kappa}_{z} \star z_{\alpha}=-z_{\alpha} \star \check{\kappa}_{z}, \quad \check{\kappa}_{z} \star \check{\kappa}_{z}=1,
$$

that is, it obeys the defining property of $\kappa_{z}$, viz. $\check{\kappa}_{z} \star f(z) \star \check{\kappa}_{z}=\pi(f(z))$. Thus, setting aside the subtler existence issue, we are led to (3.29). 
A twistor-space distribution. At first order, the $z$-dependence of $\widehat{V}^{(1) \pm}$ is captured by the distribution

$$
I^{ \pm}(z):=2 z^{ \pm} \int_{-1}^{1} \frac{d t}{(t+1)^{2}} e^{i \frac{t-1}{t+1} z^{+} z^{-}}=\frac{1}{i z^{\mp}}
$$

where we have used $\lim _{\epsilon \rightarrow 0^{+}} e^{-\frac{i}{\epsilon} z^{+} z^{-}}=0$, which follows from (3.29). It enjoys the following properties

$$
i z^{\mp} I^{ \pm}=1, \quad \partial_{ \pm} I^{ \pm}=\kappa_{z},
$$

of which the first one clearly follows from (3.34), while the second one requires more care, as $1 / z^{\mp}$ is not differentiable at $z^{\mp}=0$. Thus, in order to differentiate $I$ we must first rewrite it as a differentiable distribution, for which we use

$$
\begin{aligned}
\partial_{ \pm} I^{ \pm} & =\partial_{ \pm}\left(\int_{0}^{z^{ \pm}} d z^{ \pm} \lim _{\epsilon \rightarrow 0^{+}} \frac{1}{\epsilon} e^{-\frac{i}{\epsilon} z^{ \pm} z^{\mp}}\right) \\
& =2 \pi \partial_{ \pm}\left(\int_{0}^{z^{ \pm}} d z^{\prime \pm} \delta\left(z^{ \pm}\right) \delta\left(z^{\mp}\right)\right)=2 \pi \partial_{ \pm} \theta\left(z^{ \pm}\right) \delta\left(z^{\mp}\right)
\end{aligned}
$$

which yields the second equation in (3.35).

Breaking of manifest $\mathfrak{s l}(2, \mathbb{C})$ covariance and role of boundary conditions. A few observations on the form of the solution space described above are now in order. To begin with, we note that the factorized dependence on the $Y$ and $Z$ oscillators is instrumental to solving for the $Z$ dependence in a universal way, irrespectively of the precise form of the initial datum $\Phi^{\prime}(Y)$. The price one pays is the explicit appearance of the singular Dirac delta function element $\kappa_{z}$ as deformation term in the equations, which results, as explained above, in the necessary introduction of a spin-frame in order to integrate the equations in $Z$ space using standard distribution techniques.

As a consequence, the deformed oscillators $i$ ) cannot be written on a manifestly $\mathfrak{s l}(2, \mathbb{C})$ covariant form; and they ii) are distributions in $Z$ space, with auxiliary integral representations (3.30), taking values in the extension of the Weyl algebra in $Y$ space by the inner Kleinian $\kappa_{y}$ and its hermitian conjugate, as anticipated in section 2. Therefore, the solution in Weyl ordering is not real-analytic in $Z$, and we have shown explicitly how the poles in the $t$-integral reflect themselves into $Z$-space non-analyticities at first order. Thus, the integral form (3.33) is crucial in order to evaluate star-products involving the deformed oscillators, and it indeed yields an associative algebra; for the proof, see section 4.5.

As we shall see next, choosing the initial data to correspond to massless particles and black holes, it follows that $\Psi$ belongs to an associative algebra in which $\Psi^{\star n}$ can be rigorously defined. Moreover, upon going to normal order, the internal connection becomes real-analytic in $\mathcal{Z}$ for generic spacetime points once the vacuum gauge function $L$ is switched on. This facilitates the construction of a gauge function correction $H$ that brings the solution space to the Vasiliev gauge, at least at the linearized level, where thus Fronsdal fields arise in the asymptotic region, as we shall examine further below. 


\section{Internal solution space with scalar field and black hole modes}

In this section, we examine the exact twistor-space solution in holomorphic gauge in the case when $\Phi^{\prime}$ consists of operators with distinct eigenvalues under left and right starmultiplication by the generators of the compact Cartan subalgebra of $\mathfrak{s o}(2,3)$ [1], corresponding to linearized massless scalar modes and spherically symmetric black hole modes. Unlike in a local field theory, black hole modes arise as a back-reaction to the scalar particle modes already at second order in perturbation theory. We would like to stress that the exact solutions in twistor space can be thought of as solution to the full set of equations over a special base point in $\mathcal{X}$ where the gauge function is equal to one; the inclusion of nontrivial gauge functions will be the topic of the next section.

\subsection{Particle and black hole modes}

Extending the approach of $[1,87]$, we shall take $\Phi^{\prime}(Y)$ to belong to a reducible twistedadjoint representation space consisting of eight unitarizable irreps as follows:

$$
\mathfrak{H}^{(+)} \oplus \pi\left(\mathfrak{H}^{(+)}\right) \oplus \mathfrak{H}^{(-)} \oplus \pi\left(\mathfrak{H}^{(-)}\right),
$$

where

$$
\mathfrak{H}^{( \pm)}:=\mathfrak{D}^{( \pm)} \oplus \mathfrak{S}^{( \pm)}, \quad \mathfrak{S}^{( \pm)}:=\mathfrak{D}^{( \pm)} \star \kappa_{y},
$$

and $\mathfrak{D}^{( \pm)}$consist of operators obtained by acting with reflectors ${ }^{18}[87]$ on squares of the scalar and spinor singletons $\mathfrak{D}\left(\frac{1}{2} ;(0)\right)$ and $\mathfrak{D}\left(1 ;\left(\frac{1}{2}\right)\right)$, respectively, viz.

$$
\begin{aligned}
& \mathfrak{D}^{(-)} \stackrel{\text { refl. }}{\cong}\left[\mathfrak{D}\left(\frac{1}{2} ;(0)\right)\right]^{\otimes 2} \cong \bigoplus_{s=0,1,2, \ldots} \mathfrak{D}(s+1 ;(s)), \\
& \mathfrak{D}^{(+)} \stackrel{\text { refl. }}{\cong}\left[\mathfrak{D}\left(1 ;\left(\frac{1}{2}\right)\right)\right]^{\otimes 2} \cong \mathfrak{D}(2 ;(0)) \oplus \bigoplus_{s=1,2, \ldots} \mathfrak{D}(s+1 ;(s, 1)) .
\end{aligned}
$$

These are lowest-weight spaces with positive energies sent by $\pi$ to corresponding highestweight spaces with negative energies. The resulting eight spaces in eq. (4.1) form separate twisted-adjoint $\mathfrak{h}_{1}(4)$-orbits. The superscript $( \pm)$ refers to the eigenvalue of the involution given by the composition of the twisted-adjoint action of $(-1)^{E+J^{\prime}}$ and the parity map $P$, where $J^{\prime}$ is a spatial angular momentum chosen as to make $e_{\star}^{i \pi J^{\prime}} \star P_{r} \star e_{\star}^{-i \pi J^{\prime}}=-P\left(P_{r}\right)$, idem on $M_{0 r}$. Thus, this involution commutes with the action of the full $\mathfrak{h s}_{1}(4)$ algebra, and as consequence it splits the module (4.1) into two submodules; for further details, see [87].

\footnotetext{
${ }^{18}$ The reflector sends elements in the direct product of two supersingletons to operators acting on the supersingleton Hilbert space. The twisted-adjoint action of the higher spin algebra on such an operator is therefore reflected to the left action of the higher spin algebra on the corresponding state in the tensor product of two supersingletons, that decomposes into a sum of massless particle states via the Flato-Fronsdal theorem [20]. In particular, we shall use projectors on supersingleton states that correspond to rotationally invariant massless scalar particle states [87]. As far as parity is concerned, the reflector preserves the parity of the states in $\mathfrak{D}(1 ;(0))$, while it reverses the parity of the states in $\mathfrak{D}(2 ;(0))$. In particular, the reflector maps the rotationally invariant states, which are parity even in $\mathfrak{D}(1 ;(0))$ and parity odd in $\mathfrak{D}(2 ;(0))$, to the aforementioned projectors, all of which are even under $P$.
} 
The reference states can be taken to be the scalar ground states represented by the projectors on the scalar and spinor (anti-)singleton ground states, respectively, viz.

$$
\begin{aligned}
& \mathcal{P}_{ \pm 1}(E) \equiv \mathcal{P}_{ \pm 1 ;(0)}(E)=4 \exp (\mp 4 E) \cong\left| \pm \frac{1}{2} ;(0)\right\rangle\left\langle \pm \frac{1}{2} ;(0)\right| \\
& \mathcal{P}_{ \pm 2}(E) \equiv \mathcal{P}_{ \pm 2 ;(0)}(E)=\mp 8 \exp (\mp 4 E)(1 \mp 4 E) \cong\left| \pm 1 ;\left(\frac{1}{2}\right)\right\rangle^{i}{ }_{i}\left\langle \pm 1 ;\left(\frac{1}{2}\right)\right|,
\end{aligned}
$$

where $E$ is the anti-de Sitter energy operator and $i=1,2$ is the doublet index of the spin$1 / 2$ representation of $\mathfrak{s o}(3)$; for further details, see section 4.2 and $[1,87]$. For simplicity, we shall limit the expansion of $\Phi^{\prime}$ over $\mathfrak{D}^{( \pm)} \oplus \pi\left(\mathfrak{D}^{( \pm)}\right)$to the rank- $|n|$ supersingleton projectors $\mathcal{P}_{n}(E), n= \pm 1, \pm 2, \ldots$, given by the sum of the projectors onto the (anti-)singleton states with energy $n / 2$ and spin $|n|-1 / 2$, viz. ${ }^{19}$

$$
\mathcal{P}_{n}(E) \equiv \mathcal{P}_{n ;(0)}(E) \cong\left|\frac{n}{2} ;\left(\frac{|n|-1}{2}\right)\right\rangle^{i(|n|-1)}{ }_{i(|n|-1)}\left\langle\frac{n}{2} ;\left(\frac{|n|-1}{2}\right)\right|, \quad n= \pm 1, \pm 2, \ldots,
$$

where the notation $i(|n|-1)=i_{1} i_{2} \ldots i_{|n|-1}$ denotes $|n|-1$ symmetrized doublet indices. These projectors obey

$$
\left(E-\frac{n}{2}\right) \star \mathcal{P}_{n}(E)=0=\mathcal{P}_{n}(E) \star\left(E-\frac{n}{2}\right), \quad\left[M_{r s}, \mathcal{P}_{n}(E)\right]_{\star}=0,
$$

from which it follows that they are rotationally invariant and that their twisted-adjoint energy eigenvalues are given by $n$, i.e.

$$
E \star \mathcal{P}_{n}(E)-\mathcal{P}_{n}(E) \star \pi(E)=\left\{E, \mathcal{P}_{n}(E)\right\}_{\star}=n \mathcal{P}_{n}(E)
$$

Thus, under the twisted-adjoint action $(4.9), \mathcal{P}_{n}(E)$ behave as enveloping algebra realizations of rotationally invariant modes of an $A d S_{4}$ massless scalar field [87]. In other words, the twisted-adjoint action of $\mathfrak{s o}(2,3)$ on the $\mathcal{P}_{n}(E)$ realizes the (left) $\mathfrak{s o}(2,3)$-action on the tensor product of two super(anti-)singleton states, that gives rise to specific scalar modes, in accordance with the Flato-Fronsdal theorem [20]. Indeed, via the linearization of the second equation in (3.1), this projector provides the initial data in twistor space for the scalar-field mode in spacetime with energy $n$ and vanishing spatial angular momentum found by Breitenlohner and Freedman [100], as will be shown below in section 5.2.1.

Turning to the spaces $\mathfrak{S}^{( \pm)} \oplus \pi\left(\mathfrak{S}^{( \pm)}\right)$, these are required for the non-linear completion of the particle states in our gauge, as the interactions involve $\star$-multiplications with $\kappa_{y}$ that map the projectors associated to particle states to twisted projectors

$$
\widetilde{\mathcal{P}}_{n}:=\mathcal{P}_{n}(E) \star \kappa_{y} \cong\left|\frac{n}{2} ;\left(\frac{|n|-1}{2}\right)\right\rangle_{i(|n|-1)}{ }_{i(|n|-1)}\left\langle-\frac{n}{2} ;\left(\frac{|n|-1}{2}\right)\right|, \quad n= \pm 1, \pm 2, \ldots,
$$

by transforming singleton states into anti-singleton states with opposite energy, and viceversa. ${ }^{20}$ These elements are the initial data for static, spherically symmetric higher spin

\footnotetext{
${ }^{19}$ More precisely, in [1], the projectors $\mathcal{P}_{n}(E)$ were referred to as symmetry-enhanced, in order to distinguish them from the rank-1, biaxially-symmetric projectors $P_{n_{1} n_{2}}(E, J)$, depending on $E$ as well as an angular momentum $J$.

${ }^{20}$ As a consequence of the action of parity on the oscillators (2.55), and of the third of eqs. (4.21), the twisted projectors $\widetilde{\mathcal{P}}_{n}$ are alternatively odd or even under $P, P\left(\widetilde{\mathcal{P}}_{n}\right)=(-1)^{n} \widetilde{\mathcal{P}}_{n}$.
} 
generalizations of Schwarzschild black holes [1,3]. Indeed, following the same reasoning as above, one can show that

$$
E \star \widetilde{\mathcal{P}}_{n}-\widetilde{\mathcal{P}}_{n} \star \pi(E)=\left[E, \mathcal{P}_{n}(E)\right]_{\star} \star \kappa_{y}=0,
$$

implying that the twisted projectors give rise to static solutions. Concerning the more detailed nature of the linearized fluctation fields, we recall that while the lowest-weight space modes give rise to fields that are regular everywhere in spacetime, the black hole modes exhibit the typical singularity at $r=0$, as dictated by the spherical symmetry and the generalized Petrov Type D structure in each spin- $s$ Weyl zero-form component (see appendix A for a quick review of Petrov's classification).

Thus, starting from a linearized particle mode, the star-product interactions generate black hole modes in $\left(\widehat{V}_{\alpha}^{\prime}, \widehat{\bar{V}}_{\dot{\alpha}}^{\prime}\right)$ already at second order, and hence in the spacetime gauge fields once the gauge function is switched on. We interpret the perturbative mixing of particle and black hole modes as a manifestation of the non-locality of the interactions in the Vasiliev equations. This raises the issue of whether one can fine-tune $\Phi^{\prime}$ and the gauge function $L$ to create a branch of moduli space that is smooth at the origin of spacetime, which could correspond holographically, via suitable generating functions, to three-dimensional conformal field theories at zero temprerature; we shall comment on this open problem towards the conclusions.

Remark on more general Killing vectors. We recall that, as studied in $[1,2]$, the solution space based on $\mathfrak{D}^{( \pm)} \equiv \mathfrak{D}^{( \pm)}(E ; J)$ admits a generalization to six different inequivalent solution spaces $\mathfrak{D}^{( \pm)}(K ; \widetilde{K})$, with different physical meaning, labelled by pairs of mutually commuting and normalized elements $K$ and $\widetilde{K}$ of the Cartan subalgebra of $\mathfrak{s p}(4 ; \mathbb{C})$ taken from the set $\{E, J, i B, i P\}$, where $J$ is an angular momentum, $B$ a (hermitian) boost and $P$ a (hermitian) translation. Thus, restricting to symmetry-enhanced solutions, the construction sketched above can be repeated for the more general projectors

$$
\mathcal{P}_{n}(K), \quad K=E, J, i B, i P .
$$

The corresponding twisted-adjoint $\mathfrak{h} \mathfrak{s}(4)$ orbits give rise to linearized solutions spaces given by expansions over lowest-weight spaces that in general not unitary. In the cases that $K=E, i P$, their fully non-linear completions, which are soliton-like states, contain twisted projectors (that necessarily appear at higher orders). In the cases that $K=J, i B$, however, the $\mathcal{P}_{n}(K)$ are eigenstates of one-sided star multiplication by $\kappa_{y}$, and hence the full solutions can be given by expansions over the $\mathcal{P}_{n}(K)$ alone.

\subsection{Regular presentation of generalized projectors}

Next, as anticipated, we shall expand the initial datum $\Phi^{\prime}$, and therefore $\Psi$ and $\widehat{V}_{\alpha}^{\prime}$, in terms of projectors and twisted projectors, which we shall refer to collectively as generalized projectors. To this end, let us start by recalling that the rotationally-invariant supersingleton projector $\mathcal{P}_{n}(E)$ given in $(4.7)$ can be realized as $[1](n= \pm 1, \pm 2, \ldots)$

$$
\mathcal{P}_{n}(E)=4(-1)^{n-\frac{1+\varepsilon}{2}} e^{-4 E} L_{n-1}^{(1)}(8 E)=2(-1)^{n-\frac{1+\varepsilon}{2}} \oint_{C(\varepsilon)} \frac{d \eta}{2 \pi i}\left(\frac{\eta+1}{\eta-1}\right)^{n} e^{-4 \eta E},
$$


where $L_{n}^{(1)}(x)$ are generalized Laguerre polynomials and the contour integral is performed around a small contour $C(\varepsilon)$ encircling $\varepsilon:=n /|n|$. As for the twisted projectors, the corresponding presentation is given by

$$
\widetilde{\mathcal{P}}_{n}:=\mathcal{P}_{n}(E) \star \kappa_{y}=4 \pi(-)^{n-\frac{1+\varepsilon}{2}} \oint_{C(\varepsilon)} \frac{d \eta}{2 \pi i}\left(\frac{\eta+1}{\eta-1}\right)^{n} \delta^{2}\left(y-i \eta \sigma_{0} \bar{y}\right) .
$$

The twisted projectors are not idempotent, but rather the generalized projectors form a subalgebra of the $\star$-product algebra containing an ideal spanned by the projectors, viz.

$$
\begin{aligned}
& \mathcal{P}_{n} \star \mathcal{P}_{m}=\delta_{n m} \mathcal{P}_{n}, \\
& \mathcal{P}_{n} \star \widetilde{\mathcal{P}}_{m}=\delta_{n m} \widetilde{\mathcal{P}}_{n}, \\
& \widetilde{\mathcal{P}}_{n} \star \mathcal{P}_{m}=\delta_{n,-m} \widetilde{\mathcal{P}}_{n}, \\
& \widetilde{\mathcal{P}}_{n} \star \widetilde{\mathcal{P}}_{m}=\delta_{n,-m} \mathcal{P}_{n},
\end{aligned}
$$

where we have used the property (2.45) and performed the star products before evaluating the contour integrals. In this way, we achieve orthonormality between the $\mathcal{P}_{n}$ with positive and negative $n$ in eq. (4.15), from which eqs. (4.16)-(4.18) follow by associativity; ${ }^{21}$ see appendix $\mathrm{F}$ in [1] for a proof and a precise prescription for the contour. For this reason, we shall refer to eqs. (4.13) and (4.14) as the regular presentations of the generalized projectors.

As the nonlinear corrections are given by star-powers of the adjoint elements $\Psi$ and $\bar{\Psi}$ (see for example eq. (3.30)), we note that the fact that the product of two twisted projectors is an ordinary projector manifests the fact that free scalar modes alone do not solve the full equations, but rather their completion into full solutions includes spherically symmetric higher spin black hole modes. It is also interesting to note that the relation between free massless scalar and higher spin black hole modes is just a Fourier transform with respect to the $y$ variables, since that is what the star-multiplication with $\kappa_{y}$ amounts to [3].

\subsection{Explicit form of internal solution in holomorphic gauge}

With the purpose of generalizing the black-hole-like solutions found in [1] to solutions superposing particle and black hole modes, let us examine in more detailed the expansion of the initial datum $\Phi^{\prime}$ over projectors and twisted projectors, for which we shall use the following notation:

$$
\Phi^{\prime}=\sum_{n= \pm 1, \pm 2, \ldots}\left(\nu_{n} \mathcal{P}_{n}+\widetilde{\nu}_{n} \widetilde{\mathcal{P}}_{n}\right) \star \kappa_{y}=\sum_{n= \pm 1, \pm 2, \ldots}\left(\nu_{n} \widetilde{\mathcal{P}}_{n}+\widetilde{\nu}_{n} \mathcal{P}_{n}\right)
$$

i.e.,

$$
\Psi=\sum_{n= \pm 1, \pm 2, \ldots}\left(\nu_{n} \mathcal{P}_{n}+\widetilde{\nu}_{n} \widetilde{\mathcal{P}}_{n}\right)
$$

\footnotetext{
${ }^{21}$ Performing the auxiliary contour integrals first, on the other hand, one encounters star products between two $\delta^{2}\left(y \mp i \sigma_{0} \bar{y}\right)$ which are divergent as $y_{\alpha} \mp i\left(\sigma_{0}\right)_{\alpha}{ }^{\dot{\beta}} \bar{y}_{\dot{\beta}}$ are abelian. We shall comment on alternative perturbative schemes towards the conclusions.
} 
where $\nu_{n}$ and $\widetilde{\nu}_{n}$ are constant deformation parameters, the $\mathcal{P}_{n}$ and the $\widetilde{\mathcal{P}}_{n}$ absorb all the $Y$-dependence and obey

$$
\pi \bar{\pi}\left(\mathcal{P}_{n}\right)=\mathcal{P}_{n}=\left(\mathcal{P}_{n}\right)^{\dagger}, \quad \pi\left(\mathcal{P}_{n}\right)=\mathcal{P}_{-n}, \quad \kappa_{y} \bar{\kappa}_{\bar{y}} \star \mathcal{P}_{n}=(-1)^{n} \mathcal{P}_{n}
$$

As a consequence, the reality conditions on $\Phi$ (2.33) impose the following restrictions on the deformation parameters:

$$
\begin{aligned}
& \nu_{n}^{*}=(-1)^{n} \nu_{n}, \quad \Rightarrow \quad \nu_{n}=i^{n} \mu_{n}, \quad \mu_{n} \in \mathbb{R} \\
& \widetilde{\nu}_{n}^{*}=\widetilde{\nu}_{-n} .
\end{aligned}
$$

Thus, from (4.23) it follows that the sum over the particle states (4.19) must run symmetrically around $n=0$, such that every particle mode is accompanied by its counterpart with opposite energy.

It is now possible to evaluate the product $\Psi^{\star k}$ that appears in $\widehat{V}_{\alpha}^{\prime}$ (see eq. (3.30)) explicitly in terms of the deformation parameters. To this end, we use the generalized projector algebra (4.15)-(4.18) to write

$$
\Psi^{\star k}=\sum_{n= \pm 1, \pm 2, \ldots}\left(\nu_{n}^{(k)} \mathcal{P}_{n}+\widetilde{\nu}_{n}^{(k)} \widetilde{\mathcal{P}}_{n}\right)
$$

where $\nu_{n}^{(k)}$ and $\widetilde{\nu}_{n}^{(k)}$ are homogeneous polynomials built out of $\nu_{n}$ and $\widetilde{\nu}_{n}$, defined by

$$
\nu_{n}^{(1)}=\nu_{n}, \quad \widetilde{\nu}_{n}^{(1)}=\widetilde{\nu}_{n},
$$

and, for $k \geq 2$, by the recursive relations

$$
\begin{aligned}
& \nu_{n}^{(k)}=\nu_{n} \nu_{n}^{(k-1)}+\widetilde{\nu}_{-n} \widetilde{\nu}_{n}^{(k-1)}, \\
& \widetilde{\nu}_{n}^{(k)}=\widetilde{\nu}_{n} \nu_{n}^{(k-1)}+\nu_{-n} \widetilde{\nu}_{n}^{(k-1)} .
\end{aligned}
$$

The solution to these relations is given by

$$
\begin{aligned}
\nu_{n}^{(k)} & =\frac{1}{2^{k+1} \Gamma_{n}}\left[\left(\Delta \nu_{n}+\Gamma_{n}\right)\left(\nu_{n}+\nu_{-n}+\Gamma_{n}\right)^{k}-\left(\Delta \nu_{n}-\Gamma_{n}\right)\left(\nu_{n}+\nu_{-n}-\Gamma_{n}\right)^{k}\right], \\
\widetilde{\nu}_{n}^{(k)} & =\frac{\widetilde{\nu}_{n}}{2^{k} \Gamma_{n}}\left[\left(\nu_{n}+\nu_{-n}+\Gamma_{n}\right)^{k}-\left(\nu_{n}+\nu_{-n}-\Gamma_{n}\right)^{k}\right],
\end{aligned}
$$

where $\Delta \nu_{n}:=\nu_{n}-\nu_{-n}$ and $\Gamma_{n}:=\sqrt{\left(\Delta \nu_{n}\right)^{2}+4\left|\widetilde{\nu}_{n}\right|^{2}}$. One has

$$
\nu_{n}^{(k)}-\nu_{-n}^{(k)}=\frac{\widetilde{\nu}_{n}^{(k)}}{\widetilde{\nu}_{n}} \Delta \nu_{n}
$$

We note that the zeroes in the denominator $\Gamma_{n}$ are cancelled by zeroes in the numerators. Moreover, for every $n$ such that $\widetilde{\nu}_{n}=0$ it follows from $(4.27)$ that $\widetilde{\nu}_{n}^{(k)}=0$, i.e., the problem reduces to the one already solved in $[1,2]$, with $\nu_{n}^{(k)}=\nu_{n}^{k}, \widetilde{V}_{n, \alpha}=0$, and the particle sector disappears. 
Inserting (4.24) into (3.30), the internal connection takes the form

$$
\widehat{V}_{\alpha}^{\prime}=\sum_{n= \pm 1, \pm 2, \ldots}\left(V_{n, \alpha} \star \mathcal{P}_{n}+\widetilde{V}_{n, \alpha} \star \widetilde{\mathcal{P}}_{n}\right)
$$

and analogously for $\widehat{\bar{V}}_{\dot{\alpha}}$. Thus, the coefficients $V_{n, \alpha}$ and $\widetilde{V}_{n, \alpha}$ are given by

$$
\begin{aligned}
& V_{n, \alpha}=2 i z_{\alpha} \sum_{k=1}^{\infty}\left(\begin{array}{c}
1 / 2 \\
k
\end{array}\right)\left(-\frac{b}{2}\right)^{k} \nu_{n}^{(k)} \int_{-1}^{1} \frac{d t}{(t+1)^{2}} \frac{\left(\log \left(1 / t^{2}\right)\right)^{k-1}}{(k-1) !} e^{i \frac{t-1}{t+1} w_{z}} \\
& \widetilde{V}_{n, \alpha}=2 i z_{\alpha} \sum_{k=1}^{\infty}\left(\begin{array}{c}
1 / 2 \\
k
\end{array}\right)\left(-\frac{b}{2}\right)^{k} \widetilde{\nu}_{n}^{(k)} \int_{-1}^{1} \frac{d t}{(t+1)^{2}} \frac{\left(\log \left(1 / t^{2}\right)\right)^{k-1}}{(k-1) !} e^{i \frac{t-1}{t+1} w_{z}}
\end{aligned}
$$

with $\nu_{n}^{(k)}$ and $\widetilde{\nu}_{n}^{(k)}$ given by (4.28) and (4.29). We note that the relation (4.30) among the deformation parameters translates into

$$
V_{n, \alpha}-V_{-n, \alpha}=\frac{\Delta \nu_{n}}{\widetilde{\nu}_{n}} \widetilde{V}_{n, \alpha}
$$

This completes the solution in twistor space, i.e., in holomorphic gauge.

Alternative solution method. Another way of obtaining the internal connection, which is closer to the procedure followed in [1], is to first expand $\Phi^{\prime}$ and $\left(\widehat{V}_{\alpha}^{\prime}, \widehat{\bar{V}}_{\dot{\alpha}}^{\prime}\right)$ in generalized projectors, viz.

$$
\begin{aligned}
\Phi^{\prime} & =\sum_{n= \pm 1, \pm 2, \ldots}\left(\nu_{n} \widetilde{\mathcal{P}}_{n}+\widetilde{\nu}_{n} \mathcal{P}_{n}\right), \\
\widehat{V}_{\alpha}^{\prime} & =\sum_{n= \pm 1, \pm 2, \ldots}\left(V_{n, \alpha}(Z) \star \mathcal{P}_{n}+\widetilde{V}_{n, \alpha}(Z) \star \widetilde{\mathcal{P}}_{n}\right), \\
\widehat{\bar{V}}_{\alpha}^{\prime} & =\sum_{n= \pm 1, \pm 2, \ldots}\left(\bar{V}_{n, \dot{\alpha}}(Z) \star \mathcal{P}_{n}+\overline{\widetilde{V}}_{n, \alpha}(Z) \star \widetilde{\mathcal{P}}_{n}\right)
\end{aligned}
$$

and then insert these expansions into eqs. (3.4)-(3.6). Using the generalized projector algebra (4.15)-(4.18), the coefficients of $\mathcal{P}_{n}$ and $\widetilde{\mathcal{P}}_{n}$ in eq. (3.4) yields the two conditions

$$
\begin{aligned}
& \nu_{n}\left(V_{n, \alpha}+\pi\left(V_{n, \alpha}\right)\right)+\widetilde{\nu}_{-n} \widetilde{V}_{n, \alpha}+\widetilde{\nu}_{n} \pi\left(\widetilde{V}_{-n, \alpha}\right)=0, \\
& \widetilde{\nu}_{n}\left(V_{n, \alpha}+\pi\left(V_{-n, \alpha}\right)\right)+\nu_{-n} \widetilde{V}_{n, \alpha}+\nu_{n} \pi\left(\widetilde{V}_{n, \alpha}\right)=0,
\end{aligned}
$$

respectively. They are solved by

$$
\begin{aligned}
\pi\left(V_{n, \alpha}\right) & =-V_{n, \alpha}, \quad \pi\left(\widetilde{V}_{n, \alpha}\right)=-\widetilde{V}_{n, \alpha}, \\
V_{n, \alpha}-V_{-n, \alpha} & =\frac{\Delta \nu_{n}}{\widetilde{\nu}_{n}} \widetilde{V}_{n, \alpha} \\
\widetilde{V}_{-n, \alpha} & =e^{-2 i \varphi_{n}} \widetilde{V}_{n, \alpha},
\end{aligned}
$$

provided $\widetilde{\nu}_{n} \neq 0$, and where, recalling the reality conditions (4.23), we have defined the phase factor

$$
e^{2 i \varphi_{n}}:=\frac{\widetilde{\nu}_{n}}{\left(\widetilde{\nu}_{n}\right)^{*}}
$$


Identical considerations hold for $\widehat{\bar{V}}_{\dot{\alpha}}$. Next, eq. (3.6) gives rise to the conditions

$$
\begin{aligned}
& \partial_{\alpha} \bar{V}_{n, \dot{\alpha}}-\bar{\partial}_{\dot{\alpha}} V_{n, \alpha}+\left[V_{n, \alpha}, \bar{V}_{n, \dot{\alpha}}\right]_{\star}+\left[\widetilde{V}_{n, \alpha}, \overline{\widetilde{V}}_{-n, \dot{\alpha}}\right]_{\star}=0, \\
& \partial_{\alpha} \overline{\widetilde{V}}_{n, \dot{\alpha}}-\bar{\partial}_{\dot{\alpha}} \widetilde{V}_{n, \alpha}+\left[V_{n, \alpha}, \overline{\widetilde{V}}_{n, \dot{\alpha}}\right]_{\star}+\left[\widetilde{V}_{n, \alpha}, \bar{V}_{-n, \dot{\alpha}}\right]_{\star}=0 .
\end{aligned}
$$

Solving perturbatively in powers of $\nu_{n}, \widetilde{\nu}_{n}$, it is possible to use the gauge freedom to set to zero any non-holomorphic terms in $V_{n, \alpha}, \widetilde{V}_{n, \alpha}$, leading to the holomorphic Ansatz satisfying

$$
\begin{aligned}
\partial_{\alpha} \bar{V}_{n, \dot{\alpha}} & =0=\bar{\partial}_{\dot{\alpha}} V_{n, \alpha}, \\
\partial_{\alpha} \overline{\widetilde{V}}_{n, \dot{\alpha}} & =0=\bar{\partial}_{\dot{\alpha}} \widetilde{V}_{n, \alpha} .
\end{aligned}
$$

Finally, inserting the expansions into eq. (3.5), which is equivalent to (3.24) and (3.25), and requiring the coefficients of $\mathcal{P}_{n}$ and $\widetilde{\mathcal{P}}_{n}$ to vanish, one obtains

$$
\begin{gathered}
\partial_{[\alpha} V_{n, \beta]}+V_{n,[\alpha} \star V_{n, \beta]}+\widetilde{V}_{n,[\alpha} \star \widetilde{V}_{-n, \beta]}=-\frac{i}{4} \epsilon_{\alpha \beta} b \nu_{n} \kappa_{z} \\
\partial_{[\alpha} \widetilde{V}_{n, \beta]}+\widetilde{V}_{n,[\alpha} \star V_{-n, \beta]}+V_{n,[\alpha} \star \widetilde{V}_{n, \beta]}=-\frac{i}{4} \epsilon_{\alpha \beta} b \widetilde{\nu}_{n} \kappa_{z},
\end{gathered}
$$

which can be rewritten using the relations (4.41) and (4.42) as

$$
\begin{array}{r}
\partial_{[\alpha} V_{n, \beta]}+V_{n,[\alpha} \star V_{n, \beta]}+e^{-2 i \varphi_{n}} \widetilde{V}_{n,[\alpha} \star \widetilde{V}_{n, \beta]}=-\frac{i}{4} \epsilon_{\alpha \beta} b \nu_{n} \kappa_{z}, \\
\partial_{[\alpha} \widetilde{V}_{n, \beta]}+\widetilde{V}_{n,[\alpha} \star V_{n, \beta]}-\frac{\Delta \nu_{n}}{\widetilde{\nu}_{n}} \widetilde{V}_{n,[\alpha} \star \widetilde{V}_{n, \beta]}+V_{n,[\alpha} \star \widetilde{V}_{n, \beta]}=-\frac{i}{4} \epsilon_{\alpha \beta} b \widetilde{\nu}_{n} \kappa_{z} .
\end{array}
$$

It follows that the black hole modes of $V_{n, \alpha}$ form a closed subsector, whereas the particle modes do not. Indeed, setting $\widetilde{\nu}_{n}=0$ reduces the deformed oscillator problem (4.48)(4.49) to the one already solved in [1,2], with $\widetilde{V}_{n, \alpha}=0$ and $\nu_{n}^{(k)}=\nu_{n}^{k}$; as for the fact that black hole modes obey both the linear and the non-linear equations, see [3] and references therein. Setting $\nu_{n}=0$, on the other hand, the black hole sector resurfaces through the nonlinearities: even though the first equation has no curvature deformation term on the right-hande side, the quadratic terms in $\widetilde{V}_{n, \alpha}$ nonetheless provide a source for $V_{n, \alpha}$ as soon as one goes beyond the linearized approximation. Indeed, setting $\nu_{n}=0$ does not imply that $\nu_{n}^{(k)}$ vanishes for $k>1$, as we shall examine in detail further below. Thus, introducing a particle mode necessarily turns on the black hole sector in the $Z$-space connection $\widehat{V}_{\alpha}^{\prime}$.

We proceed by defining the normal modes

$$
\Sigma_{n, \alpha}^{[ \pm]}:=z_{\alpha}-i\left(V_{n, \alpha}+V_{-n, \alpha} \pm \frac{\Gamma_{n}}{\widetilde{\nu}_{n}} \widetilde{V}_{n, \alpha}\right),
$$

in terms which one obtains a set of two decoupled deformed-oscillator equations for every $n$ as follows:

$$
\begin{aligned}
{\left[\Sigma_{n, \alpha}^{[ \pm]}, \Sigma_{n, \beta}^{[ \pm]}\right]_{\star} } & =-2 i \epsilon_{\alpha \beta}\left(1-b M_{n}^{[ \pm]} \kappa_{z}\right) \\
\pi\left(\Sigma_{n, \alpha}^{[ \pm]}\right) & =-\Sigma_{n, \alpha}^{[ \pm]}, \quad \partial_{\dot{\alpha}} \Sigma_{n, \alpha}^{[ \pm]}=0
\end{aligned}
$$


with complex deformation parameters

$$
M_{n}^{[ \pm]}:=\frac{1}{2}\left(\nu_{n}+\nu_{-n} \pm \Gamma_{n}\right) .
$$

These equations can be solved using the refined version of the method found in [64] given in $[1,66,67]$. The solution reads

$$
\Sigma_{n, \alpha}^{[ \pm]}=4 z_{\alpha} \int_{-1}^{1} \frac{d t}{(t+1)^{2}} f_{n}^{[ \pm]}(t) e^{i \frac{t-1}{t+1} w_{z}},
$$

where $f^{n[ \pm]}(t)$ obeys the integral equations

$$
\left(f_{n}^{[ \pm]} \circ f_{n}^{[ \pm]}\right)(t)=\delta(t-1)-\frac{b M_{n}^{[ \pm]}}{2}
$$

where

$$
\left(h_{1} \circ h_{2}\right)(u):=\int_{-1}^{1} d t \int_{-1}^{1} d t^{\prime} h_{1}(t) h_{2}\left(t^{\prime}\right) \delta\left(t t^{\prime}-u\right) .
$$

Using the above integral representation, the commutator of two deformed oscillators (4.56) reproduces the singular source term using the limit representation (3.29) with parameter $\epsilon$ identified as $1+t$. For each $n$, the o-product problem is solved by

$$
\begin{aligned}
& f^{[ \pm]}(t)=\delta(t-1)+j^{[ \pm]}(t) \\
& j^{[ \pm]}(t)=q^{[ \pm]}(t)+\sum_{k=0}^{\infty} \lambda_{\sigma, k} p_{k}(t), \\
& q^{[ \pm]}(t)=-\frac{b M_{n}^{[ \pm]}}{4}{ }_{1} F_{1}\left[\frac{1}{2} ; 2 ; \frac{b M_{n}^{[ \pm]}}{2} \log \left(1 / t^{2}\right)\right]=\sum_{k=1}^{\infty}\left(\begin{array}{c}
1 / 2 \\
k
\end{array}\right)\left(-\frac{b M_{n}^{[ \pm]}}{2}\right)^{k} \frac{\left[\log \left(1 / t^{2}\right)\right]^{k-1}}{(k-1) !}
\end{aligned}
$$

where $p_{k}(t):=\frac{(-1)^{k}}{k !} \delta^{(k)}(t)$ act as projectors in the o-product algebra; fur further details as well as the explicit expression for the coefficients $\lambda_{k}$, see appendix C. Choosing $\lambda_{k}=0$, it follows from (4.52) combined with (4.41) that

$$
\begin{aligned}
V_{n, \alpha}= & i z_{\alpha} \sum_{k=1}^{\infty}\left(\begin{array}{c}
1 / 2 \\
k
\end{array}\right)\left(-\frac{b}{2}\right)^{k} \int_{-1}^{1} \frac{d t}{(t+1)^{2}} \frac{\left[\log \left(1 / t^{2}\right)\right]^{k-1}}{(k-1) !} e^{i \frac{t-1}{t+1} w_{z}} \\
& \times\left[\left(M_{n}^{[+]}\right)^{k}\left(1+\frac{\Delta \nu_{n}}{\Gamma_{n}}\right)+\left(M_{n}^{[-]}\right)^{k}\left(1-\frac{\Delta \nu_{n}}{\Gamma_{n}}\right)\right] \\
= & 2 i z_{\alpha} \sum_{k=1}^{\infty}\left(\begin{array}{c}
1 / 2 \\
k
\end{array}\right)\left(-\frac{b}{2}\right)^{k} \nu_{n}^{(k)} \int_{-1}^{1} \frac{d t}{(t+1)^{2}} \frac{\left(\log \left(1 / t^{2}\right)\right)^{k-1}}{(k-1) !} e^{i \frac{t-1}{t+1} w_{z}}
\end{aligned}
$$

and

$$
\begin{aligned}
\widetilde{V}_{n, \alpha}= & 2 i z_{\alpha} \frac{\widetilde{\nu}_{n}}{\Gamma_{n}} \sum_{k=1}^{\infty}\left(\begin{array}{c}
1 / 2 \\
k
\end{array}\right)\left(-\frac{b}{2}\right)^{k} \int_{-1}^{1} \frac{d t}{(t+1)^{2}} \frac{\left[\log \left(1 / t^{2}\right)\right]^{k-1}}{(k-1) !} e^{i \frac{t-1}{t+1} w_{z}} \\
& \times\left[\left(M_{n}^{[+]}\right)^{k}-\left(M_{n}^{[-]}\right)^{k}\right] \\
= & 2 i z_{\alpha} \sum_{k=1}^{\infty}\left(\begin{array}{c}
1 / 2 \\
k
\end{array}\right)\left(-\frac{b}{2}\right)^{k} \widetilde{\nu}_{n}^{(k)} \int_{-1}^{1} \frac{d t}{(t+1)^{2}} \frac{\left(\log \left(1 / t^{2}\right)\right)^{k-1}}{(k-1) !} e^{i \frac{t-1}{t+1} w_{z}}
\end{aligned}
$$


which coincides with the result that we had previously found in eqs. (4.32)-(4.33). We remark that the reduced deformed oscillators $\left(\Sigma_{\alpha}^{n[ \pm]}, \bar{\Sigma}_{\dot{\alpha}}^{n[ \pm]}\right)$ are real analytic away from $Z=0$ [1], where singularities in $\mathcal{Z}$ arise from the singularities of the integrand at $t=-1$; see further comments below (3.30).

\subsection{Black-hole backreaction from scalar particle modes}

In what follows, we give explicitly the solution in holomorphic gauge in the case in which $\nu_{n}=0, \forall n$, i.e., in which the Weyl zero-form contains only scalar modes. In this case formulas simplify and the backreaction mechanism producing black hole modes at higher orders becomes more transparent The resulting Ansatz for the internal master fields reads

$$
\widehat{\Phi}^{\prime}=\sum_{n \neq 0} \widetilde{\nu}_{n} \mathcal{P}_{n}, \quad \widehat{V}_{\alpha}^{\prime}=\sum_{n \neq 0} \mathcal{P}_{n} \star\left(V_{n, \alpha}+\kappa_{y} \star \widetilde{V}_{n, \alpha}\right)
$$

idem $\widehat{\bar{V}}_{\dot{\alpha}}^{\prime}$. As already noted, even if the zero-form is expanded over only projectors (and no twisted projectors), the internal connection must be expanded over both projectors and twisted projectors. At the linearized level, the presence of $\kappa_{y}$ on the right-hand side of (3.5) requires the linearized internal connection to contain only twisted projectors, but the higher order corrections to the commutator on the left-hand side of (3.5) necessarily produce both types of projectors, as is evident from eqs. (4.15)-(4.18)). More precisely, even- and odd-order corrections to the internal connection are expanded over untwisted and twisted projectors, respectively.

Proceeding, it follows from $\widehat{D}_{\alpha}^{\prime} \widehat{\Phi}^{\prime}=0$ that

$$
\begin{aligned}
\widetilde{\nu}_{n} \pi\left(\widetilde{V}_{-n, \alpha}\right)+\widetilde{\nu}_{-n} \widetilde{V}_{n, \alpha} & =0, \\
\widetilde{\nu}_{n}\left(\pi\left(V_{-n, \alpha}\right)+V_{n, \alpha}\right) & =0,
\end{aligned}
$$

with the solution

$$
\begin{aligned}
\pi\left(V_{n, \alpha}\right) & =-V_{n, \alpha}, & \pi\left(\widetilde{V}_{n, \alpha}\right) & =-\widetilde{V}_{n, \alpha}, \\
V_{-n, \alpha} & =V_{n, \alpha}, & \widetilde{V}_{-n, \alpha} & =e^{-2 i \varphi_{n}} \widetilde{V}_{n, \alpha},
\end{aligned}
$$

where the phase factor $e^{2 i \varphi_{n}}=\widetilde{\nu}_{n} /\left(\widetilde{\nu}_{n}\right)^{*}$. Moreover, the condition $\widehat{F}_{\alpha \dot{\alpha}}^{\prime}=0$ holds identically, provided that the internal connection is holomorphic, viz.

$$
\partial_{\dot{\alpha}} V_{n, \alpha}=0, \quad \partial_{\dot{\alpha}} \widetilde{V}_{n, \alpha}=0 .
$$

The deformation term in eq. (3.24) now only has components along the twisted projectors, i.e., eqs. (4.50) and (4.51) reduce to

$$
\begin{aligned}
& \partial_{[\alpha} V_{n, \beta]}+V_{n,[\alpha} \star V_{n, \beta]}+\widetilde{V}_{n,[\alpha} \star \widetilde{V}_{-n, \beta]}=0 \\
& \partial_{[\alpha} \widetilde{V}_{n, \beta]}+V_{n,[\alpha} \star \widetilde{V}_{n, \beta]}+\widetilde{V}_{n,[\alpha} \star V_{-n, \beta]}=-\frac{i b}{4} \epsilon_{\alpha \beta} \widetilde{\nu}_{n} \kappa_{z},
\end{aligned}
$$

where, as already noted, quadratic terms in $\widetilde{V}_{n, \alpha}$ source the $V_{n, \alpha}$. Defining the reduced deformed oscillators

$$
\Sigma_{n, \alpha}^{[ \pm]}:=z_{\alpha}-2 i\left(V_{n, \alpha} \pm e^{-i \varphi_{n}} \widetilde{V}_{n, \alpha}\right)
$$


one arrives at the decoupled deformed-oscillator equations for every $n$, viz.

$$
\begin{aligned}
{\left[\Sigma_{n, \alpha}^{[ \pm]}, \Sigma_{n, \beta}^{[ \pm]}\right]_{\star} } & =-2 i \epsilon_{\alpha \beta}\left(1 \mp b\left|\widetilde{\nu}_{n}\right| \kappa_{z}\right), \\
\pi\left(\Sigma_{n, \alpha}^{[ \pm]}\right) & =-\Sigma_{n, \alpha}^{[ \pm]}, \quad \partial_{\dot{\alpha}} \Sigma_{n, \alpha}^{[ \pm]}=0,
\end{aligned}
$$

where the deformation parameter indeed corresponds to $M_{n}^{[ \pm]}$evaluated at $\nu_{n}=0$. Solving these equations using the method based on integral representations spelled out above, and taking $\lambda_{k}=0$, one obtains

$$
\begin{aligned}
& V_{n, \alpha}=z_{\alpha} \frac{i b\left|\widetilde{\nu}_{n}\right|}{2} \int_{-1}^{1} \frac{d t}{(t+1)^{2}} F^{-}\left[\frac{b\left|\widetilde{\nu}_{n}\right|}{2} \log t^{2}\right] e^{i \frac{t-1}{t+1} w_{z}}, \\
& \widetilde{V}_{n, \alpha}=-z_{\alpha} \frac{i b \widetilde{\nu}_{n}}{2} \int_{-1}^{1} \frac{d t}{(t+1)^{2}} F^{+}\left[\frac{b\left|\widetilde{\nu}_{n}\right|}{2} \log t^{2}\right] e^{i \frac{t-1}{t+1} w_{z}},
\end{aligned}
$$

were we have defined the even and odd combinations

$$
F^{ \pm}[x]:=\frac{1}{2}\left({ }_{1} F_{1}\left[\frac{1}{2} ; 2 ; x\right] \pm{ }_{1} F_{1}\left[\frac{1}{2} ; 2 ;-x\right]\right) .
$$

One can indeed see, as expected, that the black hole sector $V_{n, \alpha}$ is non-vanishing beyond the leading order; it contains contributions that are of positive even orders in the deformation parameter $\widetilde{\nu}_{n}$.

We stress that the appearance of black-hole modes at second order in (classical) perturbation theory, starting from particle modes, takes place within families of exact solutions to Vasiliev's equations constructed by working with a specific set of basis functions for the master fields that facilitate the imposition of boundary and gauge conditions in spacetime as well as in the internal twistor $Z$ space. Clearly, sufficiently large changes of basis, by means of large gauge transformations or field redefinitions, may affect the perturbative organization of the master fields and hence the above phenomenon. However, one should keep in mind that the actual observables are not the master fields themselves, but rather invariant functionals thereof, and we expect an agreement at that level, noting that the perturbatively produced black-hole modes provide additional corrections to observables built from particle polarization tensors.

We note that, working within our scheme, the above effect is a consequence of the spacetime non-locality induced by the twistor space non-locality of the star products (see, for example, $[9,11])$, and not of the regularization procedure used to define the underlying associative algebra (see 4.5 for further details). ${ }^{22}$ The latter, in fact, is only responsible for setting to zero the star products of projectors $\mathcal{P}_{n}$ and $\mathcal{P}_{m}$ that have energy eigenvalues $n$ and $m$ of opposite sign. Instead, the appearance of black-hole modes is purely a consequence of the fact that: $i$ ) at the level of the Weyl zero-form, particle modes are encoded in the projectors $\mathcal{P}_{n}$ and black-hole ones in the twisted projectors $\widetilde{\mathcal{P}}_{n}=\mathcal{P}_{n} \star \kappa_{y}$ (i.e., the two

\footnotetext{
${ }^{22}$ Recently, working in a different basis, and imposing specific gauges and boundary conditions, it has been shown that Vasiliev's equations at quadratic order correspond to quasi-local cubic vertices [116]. Whether this scheme can be extended to a fully non-linear quasi-local deformed Fronsdal theory, and be related to the current scheme, remain open problems.
} 
types of modes are connected via a twistor-space Fourier transform); ii) the Ansatz we use reconstructs the deformed oscillators (hence the gauge field generating functions) in star powers of $\Phi \star \kappa_{y}$, which means that in the deformed oscillators the particles are represented by twisted projectors and the black holes by projectors; and iii) the reality conditions force the Weyl zero-form to contain for every particle mode $\mathcal{P}_{n}$ also its negative energy counterpart $\mathcal{P}_{-n}$. These facts imply that, starting from, say, a single particle mode of energy $n$ and its corresponding anti-particle mode,

$$
\Phi=\Phi_{\mathrm{pt}}=\widetilde{\nu}_{n} \mathcal{P}_{n}+\widetilde{\nu}_{-n} \mathcal{P}_{-n}
$$

the second order correction to $\widehat{V}_{\alpha}$ is proportional to

$$
\left(\Phi \star \kappa_{y}\right)^{\star 2}=\left(\widetilde{\nu}_{n} \mathcal{P}_{n}+\widetilde{\nu}_{-n} \mathcal{P}_{-n}\right) \star\left(\widetilde{\nu}_{n} \mathcal{P}_{-n}+\widetilde{\nu}_{-n} \mathcal{P}_{n}\right)=\widetilde{\nu}_{n} \widetilde{\nu}_{-n}\left(\mathcal{P}_{n}+\mathcal{P}_{-n}\right)
$$

corresponding, as recalled above, to black-hole states in the deformed oscillators, thus resulting from the star products $\mathcal{P}_{n} \star \mathcal{P}_{n}=\mathcal{P}_{n}$ and $\mathcal{P}_{-n} \star \mathcal{P}_{-n}=\mathcal{P}_{-n}$. The latter hold with or without the regularization, that is, one gets the same result also using the unregularized form of the projectors, obtained by first evaluating the contour integrals. In other words, the regularization is only responsible for setting to zero the other two mixed star products.

\subsection{Embedding into an associative algebra}

Let us demonstrate that the enveloping algebra of the internal deformed oscillator algebra forms a subalgebra of an associative algebra with well-defined star product. ${ }^{23,24}$

To this end, we first summarize the internal master fields as follows:

$$
\begin{aligned}
\Phi^{\prime} & =\sum_{n= \pm 1, \pm 2, \ldots}\left(\nu_{n} \mathcal{P}_{n}+\widetilde{\nu}_{n} \widetilde{\mathcal{P}}_{n}\right) \star \kappa_{y} \\
\widehat{S}_{\alpha}^{\prime} & =z_{\alpha}-2 i \sum_{n= \pm 1, \pm 2, \ldots}\left(V_{n, \alpha} \star \mathcal{P}_{n}+\widetilde{V}_{n, \alpha} \star \widetilde{\mathcal{P}}_{n}\right)
\end{aligned}
$$

with

$$
\begin{aligned}
& \mathcal{P}_{n}(E)=2(-1)^{n-\frac{1+\varepsilon}{2}} \oint_{C(\varepsilon)} \frac{d \eta}{2 \pi i}\left(\frac{\eta+1}{\eta-1}\right)^{n} e^{-4 \eta E} \\
& \widetilde{\mathcal{P}}_{n}(E):=\mathcal{P}_{n}(E) \star \kappa_{y}=4 \pi(-)^{n-\frac{1+\varepsilon}{2}} \oint_{C(\varepsilon)} \frac{d \eta}{2 \pi i}\left(\frac{\eta+1}{\eta-1}\right)^{n} \delta^{2}\left(y-i \eta \sigma_{0} \bar{y}\right),
\end{aligned}
$$

\footnotetext{
${ }^{23}$ While the proof is more straightforward with the solutions cast in factorized form, we would like to stress that the latter is not necessary in any way, and every result, in particular the finiteness of star products among master fields in our solution space, can be proved equally well in factorized and in fully normal-ordered form.

${ }^{24}$ We remark that the fact that the connection on a non-commutative symplectic geometry can be shifted to a deformed oscillator that belong to an adjoint section is important for constructing open Wilson lines, which are gauge invariant observables that have no analog in the commuting case.
} 
and

$$
\begin{aligned}
& V_{n, \alpha}=2 i z_{\alpha} \sum_{k=1}^{\infty}\left(\begin{array}{c}
1 / 2 \\
k
\end{array}\right)\left(-\frac{b}{2}\right)^{k} \nu_{n}^{(k)} \int_{-1}^{1} \frac{d t}{(t+1)^{2}} \frac{\left(\log \left(1 / t^{2}\right)\right)^{k-1}}{(k-1) !} e^{i \frac{t-1}{t+1} w_{z}} \\
& \widetilde{V}_{n, \alpha}=2 i z_{\alpha} \sum_{k=1}^{\infty}\left(\begin{array}{c}
1 / 2 \\
k
\end{array}\right)\left(-\frac{b}{2}\right)^{k} \widetilde{\nu}_{n}^{(k)} \int_{-1}^{1} \frac{d t}{(t+1)^{2}} \frac{\left(\log \left(1 / t^{2}\right)\right)^{k-1}}{(k-1) !} e^{i \frac{t-1}{t+1} w_{z}}
\end{aligned}
$$

where the deformation coefficients $\nu_{n}^{(k)}$ and $\widetilde{\nu}_{n}^{(k)}$ are given in (4.28)-(4.29). From the fact that the generalized projectors form a separate star product algebra, as in eqs. (4.15)(4.18), and using the self-replication formula (C.5) (see also [1]), it follows that the associative algebra generated by the internal master fields is a subalgebra of

$$
\widehat{\mathcal{A}}^{\prime}:=\left\{\sum_{n} \mathcal{P}_{n}(E) \star \sum_{i, \bar{i}, j, \bar{j}=0,1} V_{i, \bar{i}, j, \bar{j}}^{n}(z) \star \bar{V}_{i, \bar{i}, j, \bar{j}}^{n}(\bar{z}) \star\left(\kappa_{y}\right)^{\star i} \star\left(\bar{\kappa}_{\bar{y}}\right)^{\star \bar{i}} \star\left(\kappa_{z}\right)^{\star j} \star\left(\bar{\kappa}_{\bar{z}}\right)^{\star \bar{j}}\right\},
$$

where (see (C.4))

$$
\left.V_{i, \bar{i}, j, \bar{j}}^{n}:=\int_{-1}^{1} \frac{d t}{t+1} \Delta_{i, \bar{i}, j, \bar{j}}^{n}\left(t ; \partial^{(\rho)}\right) e^{\frac{i}{t+1}\left((t-1) z^{+} z^{-}+\rho^{+} z^{-}-\rho^{-} z^{+}\right.}\right)\left.\right|_{\rho=0},
$$

are defined in terms of

$$
\Delta_{i, \bar{i}, j, \bar{j}}^{n}\left(t ; \partial^{(\rho)}\right):=\sum_{p=0}^{p_{0}} f_{i, \bar{i}, j, \bar{j}}^{n, \alpha_{1} \ldots \alpha_{p}}(t) \partial_{\alpha_{1}}^{(\rho)} \cdots \partial_{\alpha_{p}}^{(\rho)},
$$

where $p_{0}$ is a finite interger, and $f_{i, i, j, \bar{j}}^{n, \alpha_{1} \ldots \alpha_{p}}(t)$ belong to the ring product algebra $\mathcal{R}$ spanned by real functions on $[-1,1]$ of the form

$$
\left(T f_{m}\right)(t):=\int_{-1}^{1} d s_{1} \cdots \int_{-1}^{1} d s_{m} f_{m}\left(s_{1}, \ldots, s_{m}\right) \delta\left(t-s_{1} \cdots s_{m}\right)
$$

with $f_{m}\left(s_{1}, \ldots, s_{m}\right)$ being a polynomial in $m$ variables. Indeed, if $T f_{m}, T g_{n} \in \mathcal{R}$, then

$$
\left(T f_{m}\right) \circ\left(T g_{n}\right)=T\left(f_{m} g_{n}\right)
$$

and $\mathcal{R}$ forms a linear space under addition and multiplication by polynomials, viz. $t^{p}\left(T f_{m}\right)(t)=T\left(\left(s_{1} \cdots s_{m}\right)^{p} f\left(s_{1}, \ldots, s_{m}\right)\right)(t)$. The star product of two elements in $\widehat{\mathcal{A}}^{\prime}$ thus involves holomorphic star products in $\mathcal{Z}$ of the form

$$
\begin{aligned}
& V(z) \star V^{\prime}(z) \\
& \left.=\int_{-1}^{1} d t \int_{-1}^{1} d t^{\prime} \frac{1}{2(\tilde{t}+1)} \Delta\left(t ; \partial^{(\rho)}\right) \Delta^{\prime}\left(t^{\prime} ; \partial^{\left(\rho^{\prime}\right)}\right) e^{\frac{i}{t+1}\left((\tilde{t}-1) z^{+} z^{-}+\tilde{\rho}^{+} z^{-}-\tilde{\rho}^{-} z^{+}-\frac{i}{2} \rho^{+} \rho^{-}+\frac{i}{2} \rho^{+} \rho^{-}\right.}\right)\left.\right|_{\rho=0=\rho^{\prime}},
\end{aligned}
$$

where $\tilde{t}=t t^{\prime}$ and $\tilde{\rho}^{ \pm}$are defined in (C.6). We can rearrange

$$
\begin{gathered}
\left.\frac{1}{2} \Delta\left(t ; \partial^{(\rho)}\right) \Delta^{\prime}\left(t^{\prime} ; \partial^{\left(\rho^{\prime}\right)}\right) e^{\frac{i}{t+1}\left(\tilde{\rho}^{+} z^{-}-\tilde{\rho}^{-} z^{+}-\frac{i}{2} \rho^{+} \rho^{-}+\frac{i}{2} \rho^{\prime+} \rho^{-}\right)}\right|_{\rho=0=\rho^{\prime}} \\
=:\left.\sum_{I} \Delta^{I}\left(t ; \partial^{(\rho)}\right) \Delta^{\prime I}\left(t^{\prime} ; \rho, \partial^{(\rho)}\right) e^{\frac{i}{\tilde{t}+1}\left(\rho^{+} z^{-}-\rho^{-} z^{+}\right)}\right|_{\rho=0},
\end{gathered}
$$


where $\Delta^{\prime I}\left(t^{\prime} ; \rho, \partial^{(\rho)}\right)=\sum_{p^{\prime}=0}^{p_{0}} \sum_{q^{\prime}=0}^{p^{\prime}} f^{I, \alpha_{1} \ldots \alpha_{p^{\prime}}, \beta_{1} \ldots \beta_{q^{\prime}}}(t) \rho_{\alpha_{1}} \cdots \rho_{\alpha_{p^{\prime}}} \partial_{\beta_{1}}^{(\rho)} \cdots \partial_{\beta_{q^{\prime}}}^{(\rho)}, \quad$ and $\left\{f^{I, \alpha_{1} \ldots \alpha_{p}}(t)\right\}$ and $\left\{f^{I I, \alpha_{1} \ldots \alpha_{p^{\prime}}, \beta_{1} \ldots \beta_{q^{\prime}}}\left(t^{\prime}\right)\right\}$ are linear combinations of $\left\{f^{\alpha_{1} \ldots \alpha_{p}}(t)\right\}$ and $\left\{f^{\prime \alpha_{1} \ldots \alpha_{p^{\prime}}}(t)\right\}$, respectively, with coefficients given by polynomials in $t$ and $t^{\prime}$; hence the new functions remain elements of $\mathcal{R}$. Thus

$$
\left.V(z) \star V^{\prime}(z)=\sum_{I} \int_{-1}^{1} \frac{d t}{t+1}\left(\Delta^{I}\left(\partial^{(\rho)}\right) \circ \Delta^{\prime I}\left(\rho, \partial^{(\rho)}\right)\right)(t) e^{\frac{i}{t+1}\left((t-1) z^{+} z^{-}+\rho^{+} z^{-}-\rho^{-} z^{+}\right.}\right)\left.\right|_{\rho=0},
$$

where

$$
\begin{aligned}
& \left(\Delta^{I}\left(\partial^{(\rho)}\right) \circ \Delta^{\prime I}\left(\rho, \partial^{(\rho)}\right)\right)(t)(\cdot) \\
:= & \sum_{p, q^{\prime}=0}^{p_{0}} \sum_{p^{\prime}=0}^{p_{0}^{\prime}}\left(f^{I, \alpha_{1} \ldots \alpha_{p}} \circ f^{I I, \beta_{1} \ldots \beta_{q^{\prime}}, \gamma_{1} \ldots \gamma_{p^{\prime}}}\right)(t) \partial_{\alpha_{1}}^{(\rho)} \ldots \partial_{\alpha_{p}}^{(\rho)}\left(\rho_{\beta_{1}} \cdots \rho_{\beta_{q^{\prime}}} \partial_{\gamma_{1}}^{(\rho)} \cdots \partial_{\gamma_{p^{\prime}}}^{(\rho)}(\cdot)\right),
\end{aligned}
$$

showing the closure of $\mathcal{A}^{\prime}$ under the star product.

\section{Spacetime dependence of the master fields}

In this section, we shall introduce different gauge functions $\widehat{g}(x, Y, Z)$ that activate the connection $\widehat{U}$ and the dependence of the master fields on $\mathcal{X}$, as to give rise to a nontrivial spacetime structure. As already stressed in section 3, these functions implement large gauge transformations, altering the asymptotics of the fields, as opposed to small, or proper, gauge transformations, which represent redundancies in the local description of the dynamics, but that can nonetheless be useful in order to remove unphysical singularities form the fields. In what follows, we shall consider three gauge functions: i) the AdS gauge function $L(x, Y)$, that takes the solution space to a gauge that we shall refer to as the L-gauge; ii) the Kruskal-like gauge function $\widehat{L}(x, Y, Z)=L(x, Y) \star \widetilde{L}(x, Z)$, where $\widetilde{L}(x, Z)$ is a local Lorentz transformation that aligns the spin frame in $\mathcal{Z}$ with that of $\mathcal{Y}$ as to remove all singularities away from the center of the black hole solutions, leading to a gauge reminiscent of the Kruskal coordinate system for the Schwarzschild solution; and iii) the Vasiliev gauge function $\widehat{G}(x, Y, Z)=L(x, Y) \star \widehat{H}(x, Y, Z)$, that takes the solution to the Vasiliev gauge where Fronsdal fields arise asymptotically in $W$ (after Lorentz covariantization and setting $Z=0$ ). We would like to stress that in the $L$-gauge, the internal connection becomes real-analytic in $\mathcal{Z}$ for generic points in $\mathcal{X}$, which facilitates the perturbative construction of $\widehat{H}$, that we shall undertake at the first order. We also note that, due to the nature of the Ansatz in section 4, linearized Fronsdal fields with $s \geqslant 1$ only arise in the black hole sector; we shall discuss the prospect of switching on these fields in the particle sector in the Conclusion.

\subsection{Gauge functions}

$\boldsymbol{A d S}_{4}$ vacuum solution. The $A d S_{4}$ spacetime provides a vacuum configuration for Vasiliev's theory, given by

$$
\widehat{\Phi}^{(0)}=0, \quad \widehat{S}_{\underline{\alpha}}^{(0)}=Z_{\underline{\alpha}}, \quad \widehat{U}^{(0)}=\Omega,
$$


where $\Omega$ is the $\mathfrak{s p}(4 ; \mathbb{R})$-valued flat connection on $\mathcal{X}$ with invertible frame field. The resulting vacuum values of $W$ and the canonical Lorentz connection $\omega$ are given by $W^{(0)}=e^{(0)}$ and $\omega^{(0)}$, where

$$
e_{\alpha \dot{\alpha}}^{(0)}=\left.2 i \lambda \frac{\partial^{2}}{\partial y^{\alpha} \partial \bar{y}^{\dot{\alpha}}} \Omega\right|_{Y=0}, \quad\left(\omega_{\alpha \beta}^{(0)}, \bar{\omega}_{\dot{\alpha} \dot{\beta}}^{(0)}\right)=\left.2 i\left(\frac{\partial^{2}}{\partial y^{\alpha} \partial y^{\beta}}, \frac{\partial^{2}}{\partial \bar{y}^{\dot{\alpha}} \partial \bar{y}^{\dot{\beta}}}\right) \Omega\right|_{Y=0},
$$

introducing the inverse AdS radius $\lambda$. A vacuum gauge function $[85,86]$ is a map

$$
L: \mathcal{R} \rightarrow \operatorname{Sp}(4 ; \mathbb{R}) / \mathrm{SL}(2 ; \mathbb{C})
$$

defined on a region $\mathcal{R} \subset \mathcal{X}$ where it obeys

$$
L^{-1} \star d L=\left.\Omega\right|_{\mathcal{R}} .
$$

The Killing symmetries, that is, the globally defined higher spin gauge transformations preserving the vacuum, have parameters given locally by

$$
\left.\widehat{\epsilon}^{(0)}\right|_{\mathcal{R}}=L^{-1} \star \epsilon^{\prime}(Y) \star L,
$$

where $\epsilon^{\prime}$ belongs to the bosonic higher spin algebra $\mathfrak{h}_{1}(4)$ or its minimal subalgebra $\mathfrak{h} \mathfrak{s}(4)$. Writing

$$
f^{L}(Y):=L^{-1}(x, Y) \star f(Y) \star L(x, Y),
$$

and defining the matrix representation $L_{\alpha \underline{\beta}}(x)$ of $L$ via

$$
Y_{\underline{\alpha}}^{L}:=L^{-1} \star Y_{\underline{\alpha}} \star L=L_{\underline{\alpha}} \underline{\beta} Y_{\underline{\beta}},
$$

it follows that

$$
f^{L}(Y)=f\left(Y^{L}\right),
$$

where Weyl ordering is assumed on both sides; in particular, we have $\widehat{\epsilon}^{(0)}=\epsilon^{\prime}\left(Y^{L}\right)$, which are globally defined Killing parameters on $A d S_{4}$.

Vacuum gauge function in stereographic coordinates. The metric can be given on manifestly Lorentz covariant form in stereographic coordinates in units where $\lambda=1$ as follows:

$$
d s_{(0)}^{2}=\frac{4 d x^{2}}{\left(1-x^{2}\right)^{2}}, \quad x^{a} \in \mathbb{R}^{4}, \quad x^{2} \neq 1,
$$

where $x^{2}:=x^{a} x^{b} \eta_{a b}$ and $d x^{2}:=d x^{a} d x^{b} \eta_{a b}$. This coordinate system provides a global cover of $A d S_{4}$, provided that the surface $x^{2}=1$ is taken to be two-sided, after which it can be identified as the boundary; for relations to embedding coordinates and spherically symmetric global coordinates, see appendix A. Correspondingly, in the notation of appendix A, we have

$$
e_{\alpha \dot{\alpha}}^{(0)}=-h^{-2}\left(\sigma^{a}\right)_{\alpha \dot{\alpha}} d x_{a}, \quad \omega_{\alpha \beta}^{(0)}=-h^{-2}\left(\sigma^{a b}\right)_{\alpha \beta} d x_{a} x_{b}, \quad h:=\sqrt{1-x^{2}} .
$$

Defining

$$
x^{a}=-\frac{1}{2}\left(\sigma^{a}\right)^{\alpha \dot{\alpha}} x_{\alpha \dot{\alpha}}, \quad x^{\alpha \dot{\alpha}}=\left(\sigma_{a}\right)^{\alpha \dot{\alpha}} x^{a}
$$


and

$$
\xi:=\left(1-h^{2}\right)^{-\frac{1}{2}} \tanh ^{-1} \sqrt{\frac{1-h}{1+h}},
$$

the vacuum connection can be integrated on

$$
\mathcal{R}=\left\{x^{a} \in \mathbb{R}^{4}: x^{2}<1\right\},
$$

and expressed in terms of the gauge function $[66,86,87]$

$$
L=\exp _{\star}\left(4 i \xi x^{a} P_{a}\right)=\frac{2 h}{1+h} \exp \frac{4 i x^{a} P_{a}}{1+h} .
$$

Its matrix representation reads

$$
L_{\underline{\alpha}} \underline{\beta}=\left(\begin{array}{cc}
\cosh (2 \xi x) \delta_{\alpha}{ }^{\beta} & \sinh (2 \xi x) \frac{x_{\alpha} \dot{\beta}}{x} \\
\sinh (2 \xi x) \frac{\bar{x}_{\dot{\alpha}}{ }^{\beta}}{x} & \cosh (2 \xi x) \delta_{\dot{\alpha}}^{\dot{\beta}}
\end{array}\right) .
$$

Kruskal-like gauge function. Following [1], we define

$$
\widehat{L}(x, Y, Z):=L(x \mid Y) \star \tilde{L}(x \mid Z), \quad \tilde{L}: \mathcal{R}_{4} \rightarrow \mathrm{SL}(2 ; \mathbb{C}) / C_{\mathrm{SL}(2 ; \mathbb{C})}\left(E^{L}\right),
$$

where $C_{\mathrm{SL}(2 ; \mathbb{C})}(M)$ denotes the subgroup of $\mathrm{SL}(2 ; \mathbb{C})$ that commutes with $M \in \mathfrak{s p}(4 ; \mathbb{C})$, and

$$
E^{L}=L^{-1} \star E \star L=-\frac{1}{8} Y^{\underline{\alpha}} Y^{\underline{\beta}} E_{\underline{\alpha \beta}}^{L}, \quad E_{\underline{\alpha \beta}}^{L}=L_{\underline{\alpha}}^{\underline{\alpha^{\prime}}} L_{\underline{\beta}} \underline{\beta^{\prime}}\left(\Gamma_{0^{\prime} 0}\right)_{\underline{\alpha^{\prime} \beta^{\prime}}} .
$$

The role of $\tilde{L}$ is to align the spin-frame on $\mathcal{Z}$ with one adapted to the $\operatorname{Sp}(4 ; \mathbb{R})$ generator $E^{L}$ used to construct the projectors $\mathcal{P}_{n}^{L}$. As shown in [1], and as we shall review below, this gauge choice removes all singularities from the gauge fields away from the spatial origin of $A d S_{4}$. In this gauge, the vacuum configuration is given by

$$
\widehat{\Phi}^{(\widehat{L})(0)}=0, \quad \widehat{S}_{\underline{\alpha}}^{(\widehat{L})(0)}=\tilde{L}^{-1} \star Z_{\underline{\alpha}} \star \tilde{L}, \quad \widehat{U}^{(\widehat{L})(0)}=\Omega+\tilde{L}^{-1} \star d \tilde{L},
$$

implying that still

$$
W^{(\widehat{L})(0)}=\Omega, \quad \omega^{(\widehat{L})(0)}=\omega^{(0)},
$$

as $\left.\tilde{L}^{-1} \star d \tilde{L}\right|_{Z=0}=0$ and $\left.\widehat{M}_{\alpha \beta}^{(\widehat{L})}\right|_{Z=0}=y_{(\alpha} \star y_{\beta)}$, though the internal connection does no longer obey the Vasiliev gauge condition.

Vasiliev gauge function. Provided that the internal connection is real analytic in $\mathcal{Z}$ for generic points on $\mathcal{X} \times \mathcal{Y}$, the Vasiliev gauge function

$$
\widehat{G}(x \mid Y, Z)=L \star \widehat{H},
$$

where $\widehat{H}$ is a perturbatively defined field-dependent large gauge transformation defined by

$$
Z^{\underline{\alpha}} S_{\underline{\alpha}}^{(\widehat{G})}=0 .
$$

We note that

$$
\widehat{H}=1+\sum_{n \geqslant 1} \widehat{H}^{(n)},
$$

and stress that $\widehat{H}^{(n)}$ may in general contain singularities in $\mathcal{C}$, as we shall exemplify further below at the linearized level in the particle sector. 


\subsection{Master fields in $L$-gauge and Kruskal-like gauge}

\subsubsection{Weyl zero-form}

From eqs. (3.1) and (4.19), and using $\pi(\widehat{L})=\pi(L) \star \tilde{L}$, it follows that

$$
\widehat{\Phi}^{(\widehat{L})}=\tilde{L}^{-1} \star \widehat{\Phi}^{(L)} \star \tilde{L}=\widehat{\Phi}^{(L)}=\Phi_{\mathrm{bh}}+\Phi_{\mathrm{pt}},
$$

where we have use the fact that $\widehat{\Phi}^{(L)}$ is $Z$-independent, and defined

$$
\Phi_{\mathrm{bh}}:=\sum_{n= \pm 1, \pm 2, \ldots} \nu_{n} \mathcal{P}_{n}^{L} \star \kappa_{y}, \quad \Phi_{\mathrm{pt}}:=\sum_{n= \pm 1, \pm 2, \ldots} \widetilde{\nu}_{n} \widetilde{\mathcal{P}}_{n}^{L} \star \kappa_{y},
$$

recalling that $\mathcal{P}_{n}^{L} \equiv L^{-1} \star \mathcal{P}_{n} \star L$ and $\widetilde{\mathcal{P}}_{n}^{L} \equiv L^{-1} \star \widetilde{\mathcal{P}}_{n} \star L$. We also define the generating functions

$$
C_{\mathrm{bh}}(x, y):=\left.\Phi_{\mathrm{bh}}\right|_{\bar{y}=0}, \quad C_{\mathrm{pt}}(x, y):=\left.\Phi_{\mathrm{pt}}\right|_{\bar{y}=0},
$$

for the dynamical scalar field and the (self-dual part of the) spin $s=1,2, \ldots$ Weyl tensors in the black hole and particle sectors, respectively. As we shall demonstrate next, all spins are activated in $C_{\mathrm{bh}}(x, y)$, whose spin-2 component is the Schwarzschild black hole Weyl tensor $[1,3]$, while $\Phi_{\mathrm{pt}}$ only consists of a rotationally-invariant dynamical scalar field.

Black-hole sector. Using the regular presentation (4.84), we have

$$
\mathcal{P}_{n}^{L} \star \kappa_{y}=2(-1)^{n-\frac{1+\epsilon}{2}} \oint_{C(\epsilon)} \frac{d \eta}{2 \pi i}\left(\frac{\eta+1}{\eta-1}\right)^{n} e^{-4 \eta E^{L}} \star \kappa_{y},
$$

where

$$
e^{-4 \eta E^{L}} \equiv L^{-1} \star e^{-4 \eta E} \star L=e^{-\frac{\eta}{2}\left(y^{\alpha} y^{\beta} \varkappa_{\alpha \beta}^{L}+\bar{y}^{\dot{\alpha}} \bar{y}^{\dot{\beta}} \bar{\varkappa}_{\dot{\alpha} \dot{\beta}}^{L}+2 y^{a} \bar{y}^{\dot{\beta}} v_{\alpha \dot{\beta}}^{L}\right)} .
$$

The matrix $v_{\alpha \dot{\beta}}^{L}$ is a Killing vector with Killing two-form $\left(\varkappa_{\alpha \beta}^{L}, \bar{\varkappa}_{\dot{\alpha} \dot{\beta}}^{L}\right)$. In terms of the global radial AdS coordinate $r$, one has

$$
\left(\varkappa^{L}\right)^{2}:=\frac{1}{2}\left(\varkappa^{L}\right)^{\alpha \beta}\left(\varkappa^{L}\right)_{\alpha \beta}=-r^{2} .
$$

By introducing an adapted spin-frame consisting of the $x$-dependent eigenspinors $\left(u_{(E) \alpha}^{+}, u_{(E) \alpha}^{-}\right)$of $\varkappa_{\alpha \beta}^{L}$, we can write

$$
\varkappa_{\alpha \beta}^{L}=r \mathcal{D}_{(E) \alpha \beta}, \quad v_{\alpha \dot{\beta}}^{L}=\sqrt{1+r^{2}} \mathcal{T}_{(E) \alpha \dot{\beta}},
$$

where

$$
\mathcal{D}_{(E) \alpha \beta}:=u_{(E) \alpha}^{+} u_{(E) \beta}^{-}+u_{(E) \alpha}^{-} u_{(E) \beta}^{+}, \quad \mathcal{T}_{(E) \alpha \dot{\beta}}:=u_{(E) \alpha}^{+} \bar{u}_{(E) \dot{\beta}}^{+}+u_{(E) \alpha}^{-} \bar{u}_{(E) \dot{\beta}}^{-} ;
$$

see [1] for further details. Performing the star product $e^{-4 \eta E^{L}} \star \kappa_{y}$ in (5.26) yields

$$
\begin{aligned}
\Phi_{\mathrm{bh}}= & \frac{2}{\sqrt{\left(\varkappa^{L}\right)^{2}}} \sum_{n= \pm 1, \pm 2, \ldots}(-1)^{n-\frac{1+\epsilon}{2}} \nu_{n} \oint_{C(\epsilon)} \frac{d \eta}{2 \pi i \eta}\left(\frac{\eta+1}{\eta-1}\right)^{n} \\
& \times \exp \left\{\frac{1}{2}\left[\frac{1}{\eta} y^{\alpha}\left(\varkappa^{L}\right)_{\alpha \beta}^{-1} y^{\beta}+\eta \bar{y}^{\dot{\alpha}}\left(\bar{\varkappa}^{L}\right)_{\dot{\alpha} \dot{\beta}}^{-1} \bar{y}^{\dot{\beta}}+2 i y^{\alpha} \bar{y}^{\dot{\beta}}\left(\varkappa^{L}\right)_{\alpha \beta}^{-1}\left(v^{L}\right)^{\beta}{ }_{\dot{\beta}}\right]\right\} .
\end{aligned}
$$


Since

$$
\left.e^{-4 \eta E^{L}} \star \kappa_{y}\right|_{\bar{y}=0}=\frac{1}{\sqrt{\left(\eta \varkappa^{L}\right)^{2}}} \exp \frac{1}{2 \eta} y^{\alpha}\left(\varkappa^{L}\right)_{\alpha \beta}^{-1} y^{\beta}, \quad\left(\varkappa^{L}\right)_{\alpha \beta}^{-1}=\frac{1}{r} \mathcal{D}_{(E) \alpha \beta},
$$

we have

$$
C_{\mathrm{bh}}(x, y)=\frac{2}{r} \sum_{n=1}^{\infty} i^{n-1} \oint_{C(1)} \frac{d \eta}{2 \pi i \eta}\left(\frac{\eta+1}{\eta-1}\right)^{n} \sum_{\epsilon= \pm 1}(-1)^{\frac{1+\epsilon}{2}(n-1)} \mu_{\epsilon n} e^{\frac{\epsilon}{2 \eta} y^{\alpha}\left(\varkappa^{L}\right)_{\alpha \beta}^{-1} y^{\beta}} .
$$

For a given $n$, the contribution from $\nu_{n} \mathcal{P}_{n}(E)$ to the spin- $s$ sector is thus given, up to an $n$ - and $s$-dependent real factor, by

$$
C_{\mathrm{bh}, n, \alpha(2 s)} \sim \frac{i^{n-1} \mu_{n}}{r^{s+1}}\left(u_{(E)}^{+} u_{(E)}^{-}\right)_{\alpha(2 s)}^{s}
$$

where we note that $i^{n-1} \mu_{n}$ is real and imaginary, respectively in the case of scalar (odd $n$ ) and spinor (even $n$ ) singleton twisted projectors. The Weyl tensors are thus of generalized Petrov type D [1], and the corresponding asymptotic charges are electric and magnetic, respectively, for scalar and spinor singletons in the A model, and vice versa in the B model. As first noted in [3] for the case $n=1$, this corresponds to an $A d S_{4}$ Schwarzschild black hole Weyl tensor together with its generalization to all integer spins.

Particle sector. In order to compute $\Phi_{\mathrm{pt}}$ in (5.23), we start from the regular presentation (4.85) of the twisted projector, which transforms under the adjoint action by $L$ into

$$
\widetilde{\mathcal{P}}_{n}^{L}(E)=4 \pi(-1)^{n-\frac{1+\varepsilon}{2}} \oint_{C(\varepsilon)} \frac{d \eta}{2 \pi i}\left(\frac{\eta+1}{\eta-1}\right)^{n} \delta^{2}\left(y^{L}-i \eta \sigma_{0} \bar{y}^{L}\right)
$$

where, in stereographic coordinates,

$$
\begin{aligned}
\delta^{2}\left(y^{L}-i \eta \sigma_{0} \bar{y}^{L}\right) & =\delta^{2}(A(x, \eta) y+B(x, \eta) \bar{y}), \quad \text { with } \\
A_{\alpha}{ }^{\beta} & =\frac{1}{\sqrt{1-x^{2}}}\left(\delta_{\alpha}{ }^{\beta}-i \eta\left(\sigma_{0} \bar{x}\right)_{\alpha}^{\beta}\right), \quad B_{\alpha}^{\dot{\beta}}=\frac{1}{\sqrt{1-x^{2}}}\left(x_{\alpha}^{\dot{\beta}}-i \eta\left(\sigma_{0}\right)_{\alpha}^{\dot{\beta}}\right) .
\end{aligned}
$$

Recalling the complex analyticity property (B.1) of the delta function in non-commutative twistor space, the star product in the second equation in (5.24) can be found to be

$$
2 \pi \delta^{2}\left(y^{L}-i \eta \sigma_{0} \bar{y}^{L}\right) \star \kappa_{y}=\frac{2 \pi}{\operatorname{det} A} \delta^{2}(\widetilde{y}) \star \kappa_{y}=\frac{1}{\operatorname{det} A} e^{i y^{\alpha} M_{\alpha} \bar{\alpha}_{\dot{\alpha}}},
$$

where

$$
\begin{aligned}
M_{\alpha}^{\dot{\beta}} & :=A_{\alpha}^{-1 \beta} B_{\beta} \dot{\beta}=f_{1}(x, \eta) x_{\alpha}{ }^{\dot{\beta}}-i f_{2}(x, \eta)\left(\sigma_{0}\right)_{\alpha}^{\dot{\beta}}, \\
f_{1} & :=\frac{1-2 i \eta x_{0}+\eta^{2}}{1-2 i \eta x_{0}+\eta^{2} x^{2}}, \quad f_{2}:=\eta \frac{1-x^{2}}{1-2 i \eta x_{0}+\eta^{2} x^{2}}, \\
\operatorname{det} A & =\frac{1-2 i \eta x_{0}+\eta^{2} x^{2}}{1-x^{2}},
\end{aligned}
$$


and we have introduced

$$
\widetilde{y}_{\alpha}:=y_{\alpha}+M_{\alpha}^{\dot{\beta}}(x, \eta) \bar{y}_{\dot{\beta}},
$$

which generate a (noncommutative) Weyl algebra for generic $x^{a}$ and $\eta$, with the notable exception $\left.\widetilde{y}_{\alpha}\right|_{x=0, \eta= \pm 1}=y_{\alpha} \mp i\left(\sigma_{0}\right)_{\alpha} \dot{\beta}_{\bar{\beta}}$, which are abelian; see Footnote 21 after eq. (4.18). Thus, we arrive at

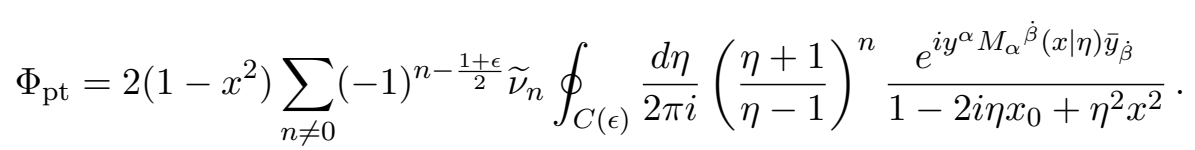

We note that the expansion in oscillators of this function only contains equal powers of $y_{\alpha}$ and $\bar{y}_{\dot{\alpha}}$, i.e., all Weyl tensors of spin $1,2,3, \ldots$ vanish and only scalar modes appear. Moreover, the reality condition on $\widehat{\Phi}$ implies that each positive-energy particle mode must be accompanied by a corresponding negative-energy anti-particle mode; see eq. (4.23). For example, if $\widetilde{\nu}_{n}=0$ for $\forall n \neq \pm 1$, then we recover the mode function of the ground state of the lowest-weight space $\mathfrak{D}(1,0)$ accompanied by its negative-energy counterpart in $\mathfrak{D}(-1,0)$, as $(5.43)$ becomes

$$
\begin{aligned}
\Phi_{\mathrm{pt}}^{|n|=1}=2\left(1-x^{2}\right) & {\left[\widetilde{\nu}_{1} \oint_{C(1)} \frac{d \eta}{2 \pi i} \frac{\eta+1}{\eta-1} \frac{e^{i y^{\alpha} M_{\alpha} \dot{\beta}(x \mid \eta) \bar{y}_{\dot{\beta}}}}{1-2 i \eta x_{0}+\eta^{2} x^{2}}\right.} \\
& \left.-\widetilde{\nu}_{-1} \oint_{C(-1)} \frac{d \eta}{2 \pi i} \frac{\eta-1}{\eta+1} \frac{e^{i y^{\alpha} M_{\alpha} \dot{\beta}(x \mid \eta) \bar{y}_{\dot{\beta}}}}{1-2 i \eta x_{0}+\eta^{2} x^{2}}\right] .
\end{aligned}
$$

The corresponding real physical scalar mode $\phi_{\mathrm{pt}}^{|n|=1}$ is given by the coefficient of the unity, i.e.

$$
\phi_{\mathrm{pt}}^{|n|=1}=4\left(\widetilde{\nu}_{1} \frac{1-x^{2}}{1-2 i x_{0}+x^{2}}+\widetilde{\nu}_{-1} \frac{1-x^{2}}{1+2 i x_{0}+x^{2}}\right)=4\left(\widetilde{\nu}_{1} \frac{e^{i t}}{\left(1+r^{2}\right)^{1 / 2}}+\widetilde{\nu}_{1}^{*} \frac{e^{-i t}}{\left(1+r^{2}\right)^{1 / 2}}\right),
$$

where the last expression is given in the global spherically symmetric coordinates, and we note that it is regular everywhere. Similarly, the scalar mode $\phi_{\mathrm{pt}}^{|n|=2}$ from the ground state of $\mathfrak{D}(2,0)$ together with its negative energy counterpart in $\mathfrak{D}(-2,0)$ can be obtained from the $\mathcal{P}_{2}$ and $\mathcal{P}_{-2}$ projectors, i.e.

$$
\phi_{\mathrm{pt}}^{|n|=2}=8\left[\widetilde{\nu}_{2}\left(\frac{1-x^{2}}{1-2 i x_{0}+x^{2}}\right)^{2}+\widetilde{\nu}_{-2}\left(\frac{1-x^{2}}{1+2 i x_{0}+x^{2}}\right)^{2}\right]=8\left[\widetilde{\nu}_{2} \frac{e^{2 i t}}{1+r^{2}}+\widetilde{\nu}_{2}^{*} \frac{e^{-2 i t}}{1+r^{2}}\right] .
$$

The projectors with $|n|>2$ encode the rotationally-invariant massless scalar modes of energy $\pm n$.

\subsubsection{Internal connection in $L$-gauge}

Applying an adjoint $L$ transformation to the internal connection (4.83), and using (5.26) and (5.35), we find

$$
\widehat{S}_{\alpha}^{(L)}=L^{-1} \star \widehat{S}_{\alpha}^{\prime} \star L=z_{\alpha}-2 i\left(\widehat{V}_{\mathrm{bh}, \alpha}^{(L)}+\widehat{V}_{\mathrm{pt}, \alpha}^{(L)}\right),
$$


where black hole sector is given by

$$
\begin{aligned}
\widehat{V}_{\mathrm{bh}, \alpha}^{(L)}= & 4 i \sum_{n}(-)^{n-\frac{1+\varepsilon}{2}} \oint_{C(\varepsilon)} \frac{d \eta}{2 \pi i}\left(\frac{\eta+1}{\eta-1}\right)^{n} \sum_{k=1}^{\infty}\left(\begin{array}{c}
1 / 2 \\
k
\end{array}\right)\left(-\frac{b}{2}\right)^{k} \nu_{n}^{(k)} \\
& \times \int_{-1}^{1} \frac{d t}{(t+1)^{2}} \frac{\left(\log \left(1 / t^{2}\right)\right)^{k-1}}{(k-1) !} e^{-4 \eta E^{L}} \star 2 i z_{\alpha} e^{i \frac{t-1}{t+1} w_{z}}
\end{aligned}
$$

and the particle sector by

$$
\begin{aligned}
\widehat{V}_{\mathrm{pt}, \alpha}^{(L)}= & 4 i \sum_{n}(-)^{n-\frac{1+\varepsilon}{2}} \oint_{C(\varepsilon)} \frac{d \eta}{2 \pi i}\left(\frac{\eta+1}{\eta-1}\right)^{n} \sum_{k=1}^{\infty}\left(\begin{array}{c}
1 / 2 \\
k
\end{array}\right)\left(-\frac{b}{2}\right)^{k} \widetilde{\nu}_{n}^{(k)} \\
& \times \int_{-1}^{1} \frac{d t}{(t+1)^{2}} \frac{\left(\log \left(1 / t^{2}\right)\right)^{k-1}}{(k-1) !} \delta^{2}\left(y^{L}-i \eta \sigma_{0} \bar{y}^{L}\right) \star z_{\alpha} e^{i \frac{t-1}{t+1} w_{z}}
\end{aligned}
$$

whose real-analyticity properties in $\mathcal{C}$ will be spelled out next.

Black-hole sector. The master field $\widehat{V}_{\mathrm{bh}, \alpha}^{(L)}$ was shown in [2] to be real-analytic in $\mathcal{T}$ except at the equatorial plane $\theta=\pi / 2$ in the spherical global coordinates of $A d S_{4}$ defined in (A.19), where singularities appear on a plane in twistor space. This can be seen by introducing a source $\rho^{\alpha}$ to write $V_{n, \alpha}$ as in (C.4), after which $\mathcal{P}_{n}^{L} \star V_{n, \alpha}$ can be computed using the lemma

$$
\begin{aligned}
& e^{-\frac{1}{2}\left(y^{\alpha} y^{\beta} \varkappa_{\alpha \beta}^{L}+\bar{y}^{\dot{\alpha}} \bar{y}^{\dot{\beta}} \bar{\varkappa}_{\dot{\alpha} \dot{\beta}}^{L}+2 y^{a} \bar{y}^{\dot{\beta}} v_{\alpha \dot{\beta}}^{L}\right)} \star e^{\frac{i}{2(t+1)}\left((t-1) z^{\alpha} z^{\beta} \mathcal{D}_{\alpha \beta}+2 \rho^{\alpha} z_{\alpha}\right)} \\
= & \frac{1}{\sqrt{\left(\varkappa^{L}\right)^{2} G^{2}}} e^{-\frac{1}{2} \bar{y}^{\dot{\alpha}} \dot{y}^{\dot{\beta}}\left(\bar{\varkappa}_{\dot{\alpha} \dot{\beta}}^{L}-\bar{v}_{\dot{\alpha}}^{L}\left(\varkappa^{L}\right)_{\alpha}^{-1 \beta} v_{\beta \dot{\beta}}^{L}\right)+\frac{i}{2(t+1)}\left((t-1) z^{\alpha} z^{\beta} \mathcal{D}_{\alpha \beta}+2 \rho^{\alpha} z_{\alpha}\right)+\frac{1}{2} b^{\alpha} b^{\beta} G_{\alpha \beta}^{-1}},
\end{aligned}
$$

where we recall that $\mathcal{D}_{\alpha \beta}:=2 u_{(\alpha}^{-} u_{\beta)}^{+}$, and

$$
\begin{aligned}
G_{\alpha \beta} & :=\left(\varkappa^{L}\right)_{\alpha \beta}^{-1}+i \frac{t-1}{t+1} \mathcal{D}_{\alpha \beta}, \\
b^{\alpha} & :=i\left[y^{\alpha}+\bar{y}^{\dot{\alpha}} \bar{v}_{\dot{\alpha}}^{L \beta}\left(\varkappa^{L}\right)_{\beta}^{-1 \alpha}+\frac{1}{t+1}\left((t-1) z^{\beta} \mathcal{D}_{\beta}^{\alpha}-\rho^{\alpha}\right)\right],
\end{aligned}
$$

and we have used $G_{\alpha \beta}^{-1}=-\frac{G_{\alpha \beta}}{G^{2}}$ with

$$
G^{2}:=\frac{1}{2} G^{\alpha \beta} G_{\alpha \beta}=\frac{(t+1)^{2}-i\left(t^{2}-1\right) \varkappa^{L \alpha \beta} \mathcal{D}_{\alpha \beta}+\left(\varkappa^{L}\right)^{2}(t-1)^{2}}{(t+1)^{2}\left(\varkappa^{L}\right)^{2}} .
$$

The crux of the matter is that, after the star product with the projectors, the singularities at $t=-1$ are moved to the zeros of $(t+1)^{2}-i\left(t^{2}-1\right) \varkappa^{L \alpha \beta} \mathcal{D}_{\alpha \beta}+\left(\varkappa^{L}\right)^{2}(t-1)^{2}$, that have imaginary parts provided that $\varkappa^{L \alpha \beta} \mathcal{D}_{\alpha \beta} \neq 0$ and real (recall that $\left(\varkappa^{L}\right)^{2}=-r^{2}$ is real), which push them away from the integration domain $t \in[-1,1]$. The quantity $\varkappa^{L \alpha \beta} \mathcal{D}_{\alpha \beta}$, which is given by the contraction of the $x$-dependent eigenspinors of $\varkappa_{\alpha \beta}^{L}(5.29)-(5.30)$ with the rigid spin-frame $\left(u_{\alpha}^{+}, u_{\alpha}^{-}\right)$, is indeed real and proportional to $\cos \theta$ : one way of proving 
this is expanding the spinors $\left(u_{(E) \alpha}^{+}, u_{(E) \alpha}^{-}\right)$over the rigid spin-frame $\left(u_{\alpha}^{+}, u_{\alpha}^{-}\right)$, thereby obtaining

$$
\varkappa^{L \alpha \beta}=r\left[2 \cos \theta u_{(\alpha}^{+} u_{\beta)}^{-}+\sin \theta\left(e^{i \phi} u_{\alpha}^{-} u_{\beta}^{-}-e^{-i \phi} u_{\alpha}^{+} u_{\beta}^{+}\right)\right] \quad \Rightarrow \quad \varkappa^{L \alpha \beta} \mathcal{D}_{\alpha \beta}=-2 r \cos \theta ;
$$

for details, see [2] and appendix E in [1]. Thus, the $t$-integral is convergent away from the equatorial plane, and one is led to the conclusion stated above.

Particle sector. To analyze the singularity structure of $\widehat{V}_{\mathrm{pt}, \alpha}^{(L)}$, we need to compute the star product in (5.49). To this end, we introduce a source $\rho_{\alpha}$ as in (C.4), and consider

$$
\begin{aligned}
& \delta^{2}\left(y^{L}-i \eta \sigma_{0} \bar{y}^{L}\right) \star e^{\frac{i}{2(t+1)}\left((t-1) z^{\alpha} z^{\beta} \mathcal{D}_{\alpha \beta}+2 \rho^{\alpha} z_{\alpha}\right)} \\
= & \frac{1}{2 \pi} \frac{1-x^{2}}{1-2 i \eta x_{0}+\eta^{2} x^{2}} \frac{t+1}{t-1} e^{\frac{i}{2} \frac{t+1}{t-1} \widetilde{y}^{\alpha} \mathcal{D}_{\alpha \beta} \widetilde{y}^{\beta}-i\left(z^{\alpha}-\frac{1}{t-1} \rho^{\beta} \mathcal{D}_{\beta}{ }^{\alpha}\right) \widetilde{y}_{\alpha}+\frac{i}{2\left(t^{2}-1\right)} \rho^{\alpha} \rho^{\beta} \mathcal{D}_{\alpha \beta},}
\end{aligned}
$$

where the modified oscillators $\widetilde{y}_{\alpha}$ are defined in (5.42), and $\left(1-x^{2}\right) /\left(1-2 i \eta x_{0}+\eta^{2} x^{2}\right)$ is regular, as shown explicitly in (5.45). The final form of $\widehat{V}_{\mathrm{pt}, \alpha}^{(L)}$ is thus given by ${ }^{25}$

$$
\begin{aligned}
\widehat{V}_{\mathrm{pt}}^{(L) \pm}= & \mp 2 i \sum_{n= \pm 1, \pm 2, \ldots}(-1)^{n-\frac{1+\varepsilon}{2}} \oint_{C(\varepsilon)} \frac{d \eta}{2 \pi i}\left(\frac{\eta+1}{\eta-1}\right)^{n} \sum_{k=1}^{\infty}\left(\begin{array}{c}
1 / 2 \\
k
\end{array}\right)\left(-\frac{b}{2}\right)^{k} \frac{\widetilde{\nu}_{n}^{(k)}}{(k-1) !} \\
& \times \frac{1-x^{2}}{1-2 i \eta x_{0}+\eta^{2} x^{2}} \widetilde{y}^{ \pm} e^{i\left(\widetilde{y}^{+} z^{-}-\widetilde{y}^{-} z^{+}\right)} \int_{-1}^{1} \frac{d t}{(t-1)^{2}}\left(\log \frac{1}{t^{2}}\right)^{k-1} e^{i \frac{t+1}{t-1} \widetilde{y}^{+} \widetilde{y}^{-}}
\end{aligned}
$$

where $\widehat{V}_{\mathrm{pt}}^{(L) \pm}:=u^{ \pm \alpha} \widehat{V}_{\mathrm{pt}, \alpha}^{(L)}$. Interestingly, the singularity in the $t$-integral has been moved from its position in the holomorphic gauge, namely at $t=-1$, to $t=+1$, unlike in the case of the black hole sector where it was removed except at the equatorial plane. ${ }^{26}$ Taking into account the more detailed structure of the poles at $t=+1$ in the exponential, it follows that $\widehat{V}_{\mathrm{pt}, \alpha}^{(L)}$ is regular in $\mathcal{Z}$, while it has singularities on a plane in $\mathcal{Y}$, given by a pole in the first order of the perturbative expansion (i.e. for $k=1$ ).

Singularity-free twistor space curvature. We remark that the singularities are cancelled, however, in the star commutator $\left[\widehat{S}^{(L)-}, \widehat{S}^{(L)+}\right]_{\star}$, which yields the source term $-2 i\left(1-b \widehat{\Phi}^{(L)} \star \kappa\right)$, which is regular (and exact already at the first order, as the Weyl zero-form does not receive any non-linear corrections in the $L$-gauge). To exhibit this, we consider the linearized equation

$$
\partial_{+} \widehat{V}_{\mathrm{pt}}^{(L)(1)+}+\partial_{-} \widehat{V}_{\mathrm{pt}}^{(L)(1)-}=\frac{b}{2 i} \Phi_{\mathrm{pt}}^{(L)} \star \kappa, \quad \partial_{ \pm}=\partial / \partial z^{ \pm},
$$

\footnotetext{
${ }^{25}$ The solutions contain two sources of explicit Lorentz symmetry breaking: one due to the expansion over the generalized projectors, and another one due to the introduction of $w_{z}$ in representing $\delta^{2}(z)$ as a delta sequence; of these two, the latter is responsible for the different signs in (5.56) in the decomposition with respect to the spin frame.

${ }^{26}$ The formula (5.55) can be obtained directly from (5.50)-(5.53) by realizing the delta function as $\delta^{2}\left(y^{L}-\right.$ $\left.i \eta \sigma_{0} \bar{y}^{L}\right)=\frac{1}{\operatorname{det} A} \delta^{2}(\widetilde{y})=\frac{1}{\operatorname{det} A} \lim _{\epsilon \rightarrow 0^{+}} \frac{1}{\epsilon} e^{-\frac{i}{\epsilon} \widetilde{y}^{+} \widetilde{y}^{-}}$, which can be cast in the form of the first factor in (5.53) with $\varkappa_{\alpha \beta}=\frac{i}{\epsilon} \mathcal{D}_{\alpha \beta}, \bar{\varkappa}_{\dot{\alpha} \dot{\beta}}=-\frac{i}{\epsilon}\left(M^{T} \mathcal{D} M\right)_{\dot{\alpha} \dot{\beta}}, v_{\alpha \dot{\beta}}=\frac{i}{\epsilon}(\mathcal{D} M)_{\alpha \dot{\beta}}$. Thus $\varkappa^{-1} \rightarrow 0$ in that limit, and the Gaussian determinant (5.53) reduces to $\lim _{\epsilon \rightarrow 0^{+}}(t+1) /(t-1-\epsilon(t+1))=(t+1) /(t-1)$.
} 
in the special case where $\widetilde{\nu}_{n}$ vanishes except for $|n|=1$, i.e.

$$
\begin{aligned}
\widehat{V}_{\mathrm{pt}}^{(L)(1) \pm}= & \pm 2 i b\left(1-x^{2}\right)\left(\left.\frac{\widetilde{\nu}_{1} \widetilde{y}^{ \pm}}{1-2 i x_{0}+x^{2}} e^{i\left(\widetilde{y}^{+} z^{-}-\widetilde{y}^{-} z^{+}\right)} \int_{-1}^{1} \frac{d t}{(t-1)^{2}} e^{i \frac{t+1}{t-1} \widetilde{y}^{+} \widetilde{y}^{-}}\right|_{\eta=+1}\right. \\
& \left.+\left.\frac{\widetilde{\nu}_{-1} \widetilde{y}^{ \pm}}{1+2 i x_{0}+x^{2}} e^{i\left(\widetilde{y}^{+} z^{-}-\widetilde{y}^{-} z^{+}\right)} \int_{-1}^{1} \frac{d t}{(t-1)^{2}} e^{i \frac{t+1}{t-1} \widetilde{y}^{+} \widetilde{y}^{-}}\right|_{\eta=-1}\right)
\end{aligned}
$$

where $\left.\widetilde{y}_{\alpha}\right|_{\eta= \pm 1}=\tilde{y}_{\alpha}+M_{\alpha} \dot{\beta}(x, \eta= \pm 1) \bar{y}_{\dot{\beta}}$. The left-hand side of (5.57) thus reads

$$
\begin{aligned}
\partial_{+} \widehat{V}_{\mathrm{pt}}^{(L)(1)+}+\partial_{-} \widehat{V}_{\mathrm{pt}}^{(L)(1)-}= & 4 b\left(1-x^{2}\right)\left(\left.\frac{\widetilde{\nu}_{1} \widetilde{y}^{+} \widetilde{y}^{-}}{1-2 i x_{0}+x^{2}} e^{i\left(\widetilde{y}^{+} z^{-}-\widetilde{y}^{-} z^{+}\right)} \int_{-1}^{1} \frac{d t}{(t-1)^{2}} e^{i \frac{t+1}{t-1} \widetilde{y}^{+} \widetilde{y}^{-}}\right|_{\eta=+1}\right. \\
& \left.+\left.\frac{\widetilde{\nu}_{-1} \widetilde{y}^{+} \widetilde{y}^{-}}{1+2 i x_{0}+x^{2}} e^{i\left(\widetilde{y}^{+} z^{-}-\widetilde{y}^{-} z^{+}\right)} \int_{-1}^{1} \frac{d t}{(t-1)^{2}} e^{i \frac{t+1}{t-1} \widetilde{y}^{+} \widetilde{y}^{-}}\right|_{\eta=-1}\right) \cdot(5.59)
\end{aligned}
$$

As for the right-hand side of (5.57), we use (5.44) to compute

$$
\begin{aligned}
\Phi_{\mathrm{pt}}^{|n|=1} \star \kappa & =4\left(1-x^{2}\right)\left(\left.\frac{\widetilde{\nu}_{1}}{1-2 i x_{0}+x^{2}} e^{i y^{\alpha} M_{\alpha} \dot{\beta} \bar{y}_{\dot{\beta}}}\right|_{\eta=+1}+\left.\frac{\widetilde{\nu}_{-1}}{1+2 i x_{0}+x^{2}} e^{i y^{\alpha} M_{\alpha} \dot{y}_{\dot{\beta}}}\right|_{\eta=-1}\right) \star \kappa \\
& =4\left(1-x^{2}\right)\left(\left.\frac{\widetilde{\nu}_{1}}{1-2 i x_{0}+x^{2}} e^{i\left(\widetilde{y}^{+} z^{-}-\widetilde{y}^{-} z^{+}\right)}\right|_{\eta=+1}+\left.\frac{\widetilde{\nu}_{-1}}{1+2 i x_{0}+x^{2}} e^{i\left(\widetilde{y}^{+} z^{-}-\widetilde{y}^{-} z^{+}\right)}\right|_{\eta=-1}\right) .
\end{aligned}
$$

Thus, eq. (5.57) is satisfied provided that

$$
\widetilde{y}^{+} \widetilde{y}^{-} \int_{-1}^{1} \frac{d t}{(t-1)^{2}} e^{i \frac{t+1}{t-1} \widetilde{y}^{+} \widetilde{y}^{-}}=\frac{1}{2 i}
$$

which holds on the grounds of the first of the properties of the distribution $I^{ \pm}$given in (3.35). This means, in particular, that $\widehat{V}_{\mathrm{pt}}^{(L)(1) \pm}$ has a pole at $\widetilde{y}^{\mp}=0$, and that, as anticipated, the latter is exactly cancelled by the commutator in (2.67) at first order.

\subsubsection{Internal connection in Kruskal-like gauge}

Applying an adjoint $\widehat{L}$ transformation to the internal connection (4.83), and using (5.26) and (5.35) and $\widehat{L}=L \star \widetilde{L}$, we find

$$
\widehat{S}_{\alpha}^{(\widehat{L})}=\widehat{L}^{-1} \star \widehat{S}_{\alpha}^{\prime} \star \widehat{L}=\tilde{L}_{\alpha}^{\beta} z_{\beta}-2 i\left(\widehat{V}_{\mathrm{bh}, \alpha}^{(\widehat{L})}+\widehat{V}_{\mathrm{pt}, \alpha}^{(\widehat{L})}\right),
$$

where the black hole and particle contributions

$$
\widehat{V}_{\mathrm{bh}, \alpha}^{(\widehat{L})}=\widetilde{L}^{-1} \star \widehat{V}_{\mathrm{bh}, \alpha}^{(L)} \star \widetilde{L}, \quad \widehat{V}_{\mathrm{pt}, \alpha}^{(\widehat{L})}=\widetilde{L}^{-1} \star \widehat{V}_{\mathrm{pt}, \alpha}^{(L)} \star \widetilde{L},
$$

with $\widehat{V}_{\mathrm{bh}, \alpha}^{(L)}$ and $\widehat{V}_{\mathrm{pt}, \alpha}^{(L)}$ given in (5.48) and (5.49), respectively. Thus, more explicitly,

$$
\begin{aligned}
\widehat{V}_{\mathrm{bh}, \alpha}^{(\widehat{L})}= & 4 i \sum_{n}(-)^{n-\frac{1+\varepsilon}{2}} \oint_{C(\varepsilon)} \frac{d \eta}{2 \pi i}\left(\frac{\eta+1}{\eta-1}\right)^{n} \sum_{k=1}^{\infty}\left(\begin{array}{c}
1 / 2 \\
k
\end{array}\right)\left(-\frac{b}{2}\right)^{k} \nu_{n}^{(k)} \\
& \times \int_{-1}^{1} \frac{d t}{(t+1)^{2}} \frac{\left(\log \left(1 / t^{2}\right)\right)^{k-1}}{(k-1) !} e^{-4 \eta E^{L}} \star 2 i z_{\alpha}^{\tilde{L}} e^{i \frac{t-1}{t+1} w_{z}^{\tilde{L}}}
\end{aligned}
$$


and

$$
\begin{aligned}
\widehat{V}_{\mathrm{pt}, \alpha}^{(\widehat{L})}= & 4 i \sum_{n}(-)^{n-\frac{1+\varepsilon}{2}} \oint_{C(\varepsilon)} \frac{d \eta}{2 \pi i}\left(\frac{\eta+1}{\eta-1}\right)^{n} \sum_{k=1}^{\infty}\left(\begin{array}{c}
1 / 2 \\
k
\end{array}\right)\left(-\frac{b}{2}\right)^{k} \widetilde{\nu}_{n}^{(k)} \\
& \times \int_{-1}^{1} \frac{d t}{(t+1)^{2}} \frac{\left(\log \left(1 / t^{2}\right)\right)^{k-1}}{(k-1) !} \delta^{2}\left(y^{L}-i \eta \sigma_{0} \bar{y}^{L}\right) \star z_{\alpha}^{\tilde{L}} e^{i \frac{t-1}{t+1} w_{z}^{\tilde{L}}},
\end{aligned}
$$

with

$$
z_{\alpha}^{\tilde{L}}:=\tilde{L}_{\alpha}{ }^{\beta} z_{\beta}, \quad u^{ \pm \beta} \tilde{L}_{\beta}{ }^{\alpha}=u_{(E)}^{ \pm \alpha}, \quad w_{z}^{\tilde{L}}:=\frac{1}{2} z^{\alpha} z^{\beta} \mathcal{D}_{(E) \alpha \beta},
$$

where these equalities follow from the definition made in (5.30) (see appendix E in [1] for the explicit form of $\tilde{L}_{\alpha}{ }^{\beta}$ ). In what follows, we shall first recall how the conjugation by $\tilde{L}$ removes unphysical singularities in $\mathcal{Z}$ in $\widehat{V}_{\mathrm{bh}}^{(L)}$, as first found in [1], and then show that it preserves the real-analyticity property of $\widehat{V}_{\mathrm{pt}}^{(L)}$ in $\mathcal{Z}$.

Black-hole sector. As observed in [1], the $\mathrm{SL}(2, \mathbb{C})$ transformation induced by $\widetilde{L}$ aligns the spin-frame in $\mathcal{Z}$ with that of $\left(u_{(E) \alpha}^{+}, u_{(E) \alpha}^{-}\right)$, as in (5.66), thereby modiying the quantities in (5.51) and (5.52). The resulting modification of the determinant in (5.53) contains the factor $(t+1)^{2}-i\left(t^{2}-1\right) \varkappa^{L \alpha \beta} \mathcal{D}_{(E) \alpha \beta}+\left(\varkappa^{L}\right)^{2}(t-1)^{2}$, the imaginary part of which only vanishes at $r=0$, which is thus the only singular point of $\widehat{V}_{\mathrm{bh} \alpha}^{(\widehat{L})}$.

As $r=0$ is the only singular point of the Weyl zero-form, we conclude that the singularities in the $L$-gauge at the equatorial plane away from $r=0$ are gauge artifacts.

Particle sector. As explained before, the singularity structure in $\mathcal{C}$ of $\widehat{V}_{\mathrm{pt}}^{(\widehat{L})}$ is related to the nonintegrable divergencies of the measure of the $t$-integral. To study the latter, we need the modified version of (5.55), viz.

$$
\begin{aligned}
& \delta^{2}\left(y^{L}-i \eta \sigma_{0} \bar{y}^{L}\right) \star e^{\frac{i}{2(t+1)}\left((t-1) z^{\alpha} z^{\beta} \mathcal{D}_{(E) \alpha \beta}+2 \rho^{\alpha} z_{\alpha}^{\tilde{L}}\right)} \\
= & \frac{1}{2 \pi} \frac{1-x^{2}}{1-2 i \eta x_{0}+\eta^{2} x^{2}} \frac{t+1}{t-1} e^{\frac{i}{2} \frac{t+1}{t-1} \widetilde{y}^{\alpha} \mathcal{D}_{(E) \alpha \beta} \widetilde{y}^{\beta}-i\left(z^{\alpha}-\frac{1}{t-1} \rho^{\gamma} \mathcal{D}_{\gamma}{ }^{\beta} \tilde{L}_{\beta}{ }^{\alpha}\right) \widetilde{y}_{\alpha}+\frac{i}{2\left(t^{2}-1\right)} \rho^{\alpha} \rho^{\beta} \mathcal{D}_{\alpha \beta} .}
\end{aligned}
$$

Thus, comparing to (5.55), we conclude that $\widehat{V}_{\mathrm{pt}, \alpha}^{(\widehat{L})}$ is real-analytic in $\mathcal{Z}$ while it has singularities on a plane in $\mathcal{Y}$ depending on $\mathcal{X}$ (that is a modification of the singular plane in $L$-gauge).

We remark that as far as the real-analyticity property of $\widehat{V}_{\mathrm{pt}}^{(L)}$ in $\mathcal{Z}$ is concerned, one may argue as follows: unlike in the black hole sector, in the $L$-gauge, the star product in (5.55) does not generate any imaginary part, or any other sort of contribution that pushes the singular points of the $t$-measure out of the integration domain, independently of whether the spin-frames in $\mathcal{Z}$ and $\mathcal{Y}$ are collinear or not (point-wise over $\mathcal{X}$ ). However, the details of the singular plane in $\mathcal{Y}$ requires the detailed calculation in (5.67).

\subsection{Vasiliev gauge}

Let us investigate the mechanism whereby the (large) gauge transformation, with gauge function $\widehat{G}=L \star \widehat{H}$, that brings the solution spaces from the holomorphic gauge to the 
Vasiliev gauge, defined by the condition

$$
z^{\alpha} \widehat{V}_{\alpha}^{(\widehat{G})}+\text { h.c. }=z^{+} \widehat{V}^{(\widehat{G})-}-z^{-} \widehat{V}^{(\widehat{G})+}+\text { h.c. }=0,
$$

removes singularities in $\mathcal{T}$ in the internal connection and restores the manifest Lorentz covariance, broken by the introduction of the delta sequence (3.29).

Linearized analysis. At linearized level, the gauge transformation reads

$$
\widehat{V}_{\alpha}^{(\widehat{G})(1)}=\widehat{V}_{\alpha}^{(L)(1)}+\partial_{\alpha} \widehat{H}^{(1)} .
$$

Contracting by $z^{\alpha}$ and using that, by definition, $Z^{\underline{\alpha}} \widehat{V}_{\underline{\alpha}}^{(\widehat{G})}=0$, one finds

$$
\widehat{H}^{(1)}=-\left(\frac{1}{z^{\beta} \partial_{\beta}} z^{\alpha} \widehat{V}_{\alpha}^{(L)(1)}+\text { h.c. }\right) \text {. }
$$

Inserting $\widehat{H}^{(1)}$ into (5.69) and decomposing using the spin-frame, yields

$$
\widehat{V}^{(\widehat{G})(1) \pm}=\widehat{V}^{(L) \pm}-\partial^{ \pm} \frac{1}{z^{+} \partial_{+}+z^{-} \partial_{-}}\left(z^{+} \widehat{V}^{(L)(1)-}-z^{-} \widehat{V}^{(L)(1)+}\right) .
$$

In the black hole sector, the resulting $t$-integral was analyzed in [91], and shown to be a real-analytic function on $\mathcal{C}$ for $r>0$, and to reproduce

$$
\widehat{V}_{\mathrm{bh}, \alpha}^{(\widehat{G})(1)}=z_{\alpha} \int_{0}^{1} d t t \Phi_{\mathrm{bh}}(-t z, \bar{y}) e^{i t y^{\alpha} z_{\alpha}}
$$

that is, the linearized internal connection obtained by direct integration in the Vasiliev gauge, recalling that the linearized Weyl zero-form is the same in the $L$ and Vasiliev gauges; see, for example, $[8,84]$. At $r=0$ the internal connection is instead singular, as expected. Examining the case $\nu_{n}=0, \forall n \neq 1$, for concreteness, it reads

$$
\left.\widehat{V}_{\mathrm{bh}, \alpha}^{(\widehat{G})(1)}\right|_{r=0}=\left.z_{\alpha} \int_{0}^{1} d t t \Phi_{\mathrm{bh}}(-t z, \bar{y})\right|_{r=0} e^{i t y^{\alpha} z_{\alpha}},
$$

and, inserting $\left.\Phi_{\mathrm{bh}}(-t z, \bar{y})\right|_{r=0}=\nu_{1}\left(\mathcal{P}_{1} \star \kappa_{y}\right)(-t z, \bar{y})=\nu_{1} \delta^{2}\left(-t z-i \sigma_{0} \bar{y}\right)$, we get

$$
\left.\widehat{V}_{\mathrm{bh}, \alpha}^{(\widehat{G})(1)}\right|_{r=0}=-i \nu_{1}\left(\sigma_{0} \bar{y}\right)_{\alpha} e^{-4 E} \int_{0}^{1} d t \delta^{2}\left(t z+i \sigma_{0} \bar{y}\right),
$$

which has singularites on a plane in twistor space.

Turning to the linearized particle sector, upon defining

$$
\widetilde{u}:=\widetilde{y}^{\alpha} z_{\alpha}=\widetilde{y}^{+} z^{-}-\widetilde{y}^{-} z^{+},
$$

we compute

$$
\widehat{H}_{\mathrm{pt}}^{(1)}=-\left.\frac{i b \widetilde{\nu}_{1}}{4} \frac{1-x^{2}}{1-2 i x_{0}+x^{2}} \frac{1}{\widetilde{y}^{+} \widetilde{y}^{-}} \frac{\widetilde{y}^{+} z^{-}+\widetilde{y}^{-} z^{+}}{\widetilde{u}}\left(e^{i \widetilde{u}}-1\right)\right|_{\eta=+1}+\left.\operatorname{idem}\right|_{\eta=-1},
$$


which is regular in $\mathcal{Z}$ but has a pole in $\mathcal{Y}$. The appearance of a pole in $\mathcal{Y}$ in $\widehat{H}^{(1)}$ can be traced back to the fact that the curvature deformation $\Psi$ that builds up the internal connection is a $\delta$-function in the particle sector (4.14). It follows that

$$
\widehat{V}^{(\widehat{G})(1) \pm}=-\frac{b \widetilde{\nu}_{1}}{2} \frac{1-x^{2}}{1-2 i x_{0}+x^{2}} \frac{z^{ \pm}}{\widetilde{u}}\left[e^{i \widetilde{u}}-\frac{e^{i \widetilde{u}}-1}{i \widetilde{u}}\right]+\left.\operatorname{idem}\right|_{\eta=-1},
$$

which is indeed real-analytic everywhere on $\mathcal{C}$. Moreover, in the Vasiliev gauge the manifest Lorentz covariance is restored, and we can write

$$
\begin{aligned}
\widehat{V}_{\alpha}^{(\widehat{G})} & =-\frac{b \widetilde{\nu}_{1}}{2} \frac{1-x^{2}}{1-2 i x_{0}+x^{2}} \frac{z_{\alpha}}{\widetilde{u}}\left[e^{i \widetilde{u}}-\frac{e^{i \widetilde{u}}-1}{i \widetilde{u}}\right]+\left.\operatorname{idem}\right|_{\eta=-1} \\
& =z_{\alpha} \int_{0}^{1} d t t \Phi(-t z, \bar{y}) e^{i t y^{\alpha} z_{\alpha}}
\end{aligned}
$$

in agreement with direct integration in the Vasiliev gauge, just as in the black hole case.

Vasiliev gauge beyond the linearized approximation. So far, we have set up a perturbative scheme that provides solutions in Vasiliev gauge up to first order. We will first discuss the prospects of extending the solutions to the Vasiliev gauge to all order. Then we will propose a correspondence to an alternative perturbative scheme based on normal order on the quasi-local branch of the theory, and that these two equivalent schemes are reproducing the deformed Fronsdal theory on-shell.

The singular nature of $\widehat{H}_{\mathrm{pt}}^{(1)}$ in $\mathcal{Y}$ raises the issue of whether the master fields in the Vasiliev gauge, which are elements in $\Omega\left(\mathcal{T}^{\prime}\right)$, can be mapped to $\Omega(\mathcal{T})$, as required by the assumptions made in section 2 in order to ensure the existence of invariants based on integrals over $\mathcal{B}$ and traces over the extended Weyl algebra $\mathcal{W}$. Two related issues are whether the gauge function $\widehat{G}$ is large, that is, affects the values of invariants, and whether it induces redefinitions of the inital data for the Weyl zero-form and spacetime one-form in the $L$-gauge, which may be related to those recently proposed by Vasiliev in order to obtain a quasi-local perturbation theory in terms of Fronsdal fields [95].

Let us outline the pending steps in somewhat more detail. We thus start from the initial family $\widehat{f}_{\nu, \tilde{\nu}}^{\prime}$ of exact solutions in holomorphic gauge, built from generalized Fock space operators using parameters $\nu$ and $\tilde{\nu}$ corrresponding to black hole modes and massless particle modes, respectively, which can be given equally well in Weyl and normal order. Letting $\widehat{S}$ denote Kontsevich gauge transformations, which act on horizontal sections of $\mathcal{C}$, and $\widehat{f}$ the families of master fields making up the exact solution spaces, we would like to establish the following sequence of large Kontsevich gauge transformations:

$$
\left.\left.\widehat{f}_{\nu, \tilde{\nu}}^{\prime} \stackrel{S_{0} \circ S_{L}}{\longrightarrow} \widehat{f}_{\nu, \tilde{\nu}}^{(L)} \stackrel{S_{H_{\mu}}}{\longrightarrow} \widehat{f}_{\nu, \tilde{\nu} ; \mu}^{(\widehat{G})}\right|_{\Omega\left(\mathcal{T}^{\prime}\right)} \stackrel{\left(S_{0}\right)^{-1}}{\longrightarrow} \widehat{f}_{\nu, \tilde{\nu} ; \mu}^{(\widehat{G})}\right|_{\Omega(\mathcal{T})}
$$

where

$S_{L}$ implements the gauge transformation with gauge function $L: \mathcal{X} \rightarrow G$;

$S_{0}$ denotes the map from Weyl to normal order; 
$S_{H_{\mu}}$ implements the gauge transformation that bring the solution to Vasiliev's gauge, which fixes the gauge function $H_{\mu}$ up to a homogenous solution parametrized by a large gauge function $M_{\mu}: \mathcal{X} \rightarrow G$.

If the map exists, the final configuration $\left.\widehat{f}_{\nu, \tilde{\nu} ; \mu}^{(\widehat{G})}\right|_{\Omega(\mathcal{T})}$ is thus a set of forms on $\mathcal{B}$ valued in $\mathcal{W}$, for which we can compute an on-shell action as a functional of asymptotic data and invariant quantities. Thus, denoting the on-shell action by $S[\widehat{f}]=\int_{\mathcal{B}} \operatorname{Tr} \mathcal{W} \widehat{\mathcal{L}}(\widehat{f})$, where $\widehat{f}$ collectively denotes the master fields, we have

$$
S\left[\left.\widehat{f}_{\nu, \tilde{\nu} ; \mu}^{(\widehat{G})}\right|_{\Omega(\mathcal{T})}\right]=\int_{\mathcal{B}} \operatorname{Tr}_{\mathcal{W}}\left(\widehat{\mathcal{L}} \circ\left(S_{0}\right)^{-1} \circ S_{H_{\mu}} \circ S_{0} \circ S_{L}\right)\left(\widehat{f}_{\nu, \tilde{\nu}}^{\prime}\right) .
$$

We note that the moduli (boundary states) entering via $M_{\mu}$ can be transferred from $H_{\mu}$ to $L$ by defining $\check{H}_{\mu}=\left(M_{\mu}\right)^{-1} \star H_{\mu}$ and $L_{\mu}:=L \star M_{\mu}$, which is thus a gauge function including a boundary state.

As for the existence of the map, a nontrivial compatibility condition arises already at the second order as follows: $\widehat{H}^{(1)}$ induces a second order correction $\widehat{\Phi}^{(2)}$ to the Weyl zero-form, whose $Z$-dependent piece cannot be corrected using any second-order initial data for $\Phi^{\prime}$. Thus, the $Z$-dependent part of $\left(S_{0}\right)^{-1}\left(\widehat{\Phi}^{(\widehat{G})(2)}\right)$ must be an element of $\Omega(\mathcal{T})$, that is, upon expanding it in the basis of $\mathcal{W}$, the resulting component fields should be elements of $L^{1}(\mathcal{Z})$. The simplest realization of this condition would be a Weyl zero-form that approaches a constant value at the point at infinity of $\mathcal{Z}$, but more generally it may turn out to be necessary to allow integrable divergencies. Proceeding to higher orders, it would also be interesting to examine to what extent the compatibility may actually fix the precise form of the Vasiliev gauge condition beyond leading order; if so, then this would provide an intrinsic method for fixing this apparent ambiguity currently plaguing the theory.

\section{Conclusions and outlook}

In what follows, we first summarize our results, after which we turn to omitted details, physical interpretations and prospects for future research.

Extending the methods of $[1,2]$, in this paper we have obtained a solution space to Vasiliev's four-dimensional higher spin gravity by superposing spherically-symmetric black hole and scalar particle modes. Interestingly enough, in the gauges we use we can observe that the scalar modes give rise to a backreaction in the form of black holes modes, that arises already at the second of classical perturbation theory. This effect, unusual from the point of view of ordinary gravitational theories, can be interpreted as a consequence of the non-locality of the vertices extracted from Vasiliev's equations at every perturbative order.

Our solution method combines large gauge functions with the Ansatz in eqs. (3.15)(3.17), based on separation of twistor space variables and auxiliary integral presentations of intial data. More precisely, our construction involves:

i) The large gauge function $\widehat{G}=L \star \widehat{H}$, where $L$ creates the asymptotic anti-de Sitter region, and $\widehat{H}$ restores Vasiliev's gauge, in which the asymptotic field configurations 
consist of unfolded (free) Fronsdal fields. We have determined the linearized contribution $\widehat{H}^{(1)}$, which is regular on twistor space away from the origin for black hole modes, and singular in the twistor fiber space for particle modes. We have also studied the field configurations to all orders in the $L$-gauge, reached from the holomorphic gauge via $L$ alone, and in a Kruskal-like gauge, reached via a further local $\operatorname{SL}(2, \mathbb{C})$-rotation of the $Z$ oscillators. In such gauges the Weyl zero-form is first-order exact, and the interpretation of its content in terms of black-hole and particle modes, barring all the subtleties mentioned in the Introduction, more transparent.

ii) Prior to switching on the gauge function, the field configurations are localized to twistor space in a holomorphic gauge, where they consist of an undeformed Weyl zero-form and a deformed twistor space connection, obeying a deformed oscillator algebra whose enveloping algebra is a subalgebra of the star product algebra $\widehat{\mathcal{A}}^{\prime}$ in (4.88).

iii) The algebra $\widehat{\mathcal{A}}^{\prime}$ is expanded over an algebra of projectors and twisted projectors in supersingleton spaces realized as functions on $\mathcal{Y}$ using a regular presentation that implements the normalization of states in compact weight spaces.

iv) In the holomorphic gauge, the Weyl zero-form is constant on $\mathcal{Z}$, while the deformed oscillator is a distribution on $\mathcal{Z}$ presented via a generalized Laplace transformation, whose exterior derivative reproduces the inner Klein operator in the two-form curvature.

The construction above remains to be completed in three main respects:

a) Extension as to include general particle and black hole modes in the deformed oscillator algebra, to which we see no obstacles.

b) Computation of the gauge fields to first order, and verification of the central on-massshell theorem.

c) Computation of $\widehat{H}$ and the spacetime gauge fields to higher orders, which requires an extension of Vasiliev's gauge, and the study of whether there exists a quasi-local branch of moduli space in which the spacetime gauge fields remain smooth at the origin for nontrivial particle modes.

More generally, we would like to apply the Ansatz to more general initial data, such as for example the generalized projectors built via the different inequivalent choices of the Cartan generators of $\mathfrak{s p}(4 ; \mathbb{C})$ listed in $[1]$, which will give rise to more general Type-D solution spaces with fluctuations. 
Concerning the interpretations of our results, and future directions, a number of remarks are in order:

Black hole microstates. It was observed in [87] that the Flato-Fronsdal theorem, which states that the massless particle spectrum is contained in the direct product of two supersingletons, has a natural generalization to the direct product of a supersingleton and an anti-supersingleton, which decomposes under $\mathfrak{s o}(2,3)$ into compact weights filling up the adjoint representation of the higher spin algebra. We would like to associate the latter states to the black hole sector. To this end, we begin by recalling from [87] that reflection of one of the two spaces in the direct product to the dual space, turns these two representations into operators on which the higher spin algebra acts in the twisted adjoint fashion, that is, one should think of them as being terms in a twisted-adjoint zero-form. Moreover, both of these representations admit two dual pairs of bases, with Lorentz covariant and compact bases, connected by means of harmonic expansion. In the former basis, all basis elements are operators that are polynomial in $\mathcal{Y}$. Thus, the aforementioned association requires that in the free theory limit, the generalized Type D sector of a Fronsdal field of Lorentz spin $s$ contains the (complexified) $\mathfrak{s o}(2,3)$ irrep with highest weight $(s-1, s-1)$; see [114]. Turning to the compact bases, indeed, the massless spectrum remains spanned by basis vectors that are real-analytic, with reference state given by the Gaussian element $\mathcal{P}_{1}$, while the reference state of the adjoint representation is given by the twisted projector $\widetilde{\mathcal{P}}_{1} \sim \delta^{2}\left(y-i \sigma_{0} \bar{y}\right)$.

We would like to stress that the particle and black hole spaces are isomorphic as complex vector spaces; indeed, the isomorphism is implemented by means of one-sided star product multiplication with the inner Klein operator $\kappa_{y}$, that is, by a twistor space Fourier transform. However, as far as the real structure as higher spin modules is concerned, there is a dichotomy, as it must be imposed using the twisted-adjoint reality condition. It follows that particle states are realized in terms of complex operators, whose complex conjugates are the realizations of anti-particles, while the black hole states form real vector spaces; this can be made more manifest by looking at the adjoint $\Psi$ fields, which are hermitian in the black hole sector and twisted-hermitian in the particle sector. Thus, the particle sector is a complex vector space with a positive definite sesquilinear form (which one can realize using a suitable extension of the supertrace operation to the extended Weyl algebra [107]), i.e. a Hilbert space, while the black hole sector is real vector space with positive definite bilinear form. The correspondence between star product algebras and free conformal fields $[19,115,117]$ suggests that the $\mathbb{Z}_{2}$ symmetry between the modules embedded into the oscillator algebra has a holographic counterpart, whereby the free conformal field theory at the boundary of anti-de Sitter spacetime, with its Hilbert space structure, can be mapped by a $\mathbb{Z}_{2}$ transformation to another theory that we propose to associate to the black holes (or, rather, to the black-hole microstates, as discussed in the Introduction), with its Euclidean structure.

Having obtained a classical moduli space consisting of particle and black hole modes, it would be desirable to provide it with a free energy (or on-shell action) expressible as a higher-spin gauge-invariant functional of the particle and black-hole deformation pa- 
rameters. From it, a number of physically interesting quantities can be obtained, such as holographic correlation functions obtained from multi-particle states, and derivation of thermodynamical relations for black holes from various ensembles constructed by including off-diagonal operators into $\Phi_{\mathrm{bh}}$ and multi-black hole solutions (for which we have reason to expect that there exist a "dilute gas" approximation at large distances $[1,18]$ ). We recall that, as noted above, the gauge invariant characterization of single black hole microstates is in terms of the parameters $\nu_{n}$, i.e. the eigenvalues of $\Psi_{\mathrm{bh}}$, which are indeed independent variables. On the other hand, the asymptotic charges $\mathcal{M}_{s}$ only form independent variables in the free theory, i.e. the physical characterizations of black hole microstates in terms of individual (linearized) Weyl tensors is reliable only asymptotically.

Large nature of $\widehat{\boldsymbol{H}}$. In terms of the gauge field equations, the role of $\widehat{H}_{\mathrm{bh}}^{(1)}$ is essentially to rotate the spin- $s$ fields from the $L$-gauge to the Fronsdal frame. At the level of the Fronsdal fields, this amounts to a generalized Weyl rescaling that mixes Fronsdal tensors of different ranks and gradients of the physical scalar. In other words, in the black hole sector, we can interpret both the $L$-gauge and the Vasiliev gauge as two different frames for Fronsdal fields, of which the Vasiliev frame is preferred as it is diagonalized.

In the particle sector, on the other hand, one simply cannot expand the master fields around $Y=0$, since there are inverse powers of $Y$ in the internal connection and hence in the generating function for the gauge fields. In other words, in the particle sector the linearized transformation from $L$-gauge to Vasiliev gauge has no interpretation in terms of the original Fronsdal field content. Instead, it would be interesting to introduce an enlarged class of functions on $Y$-space, and interpret the equations of motion in $L$-gauge directly in terms of the corresponding enlarged set of component fields, thus containing the original set of Fronsdal fields as a subset.

The identification of the asymptotic charges requires the large transformation connecting the holomorphic gauge to the standard Vasiliev gauge. At first order, we have found these transformations in this paper. Interestingly, in the particle sector, the gauge function $\widehat{H}^{(1)}$ inherits a singularity in $\mathcal{Y}$ from the twistor space connection, while the master fields in Vasiliev's gauge are completely regular. The origin of the singularity in $\widehat{H}^{(1)}$ is intimately related to the holomorphic gauge we start from, in which the two-form curvature has a $\delta$-function source in twistor space. It is expected that, extending Vasiliev's gauge to higher orders, $\widehat{H}$ induce a redefinition of the initial data for the zero-form and for the spacetime one-form, to be compared with those recently proposed by Vasiliev in order to rewrite the non-linearities encoded in the equations in terms of current interactions [95] (see also [96, 97] for recent holographic tests of the local second-order interaction terms found in [95]). We plan to assess how large the gauge transformation $\widehat{H}$ is, i.e., how far the $L$ - and Vasiliev gauge are from each other, by examining its effect on observables. As a first step, it is natural to examine zero-form charges, that is, decorated open Wilson lines in $\mathcal{Z}$ [106], which are known to contain higher spin amplitudes in their leading order.

Why the Vasiliev gauge? We have obtained different solution spaces to classical equation of motion in different gauges that are presumably far from each other. Each solution space may have its own set of classical observables (finite higher spin invariants); for 
example, the holomorphic gauge supports zero-form charges, but gives trivial results for observables that involve spacetime gauge fields. Thinking of the classical theory as a saddle point of a path integral adds one more condition, namely the finiteness of a classical action on-shell, which can then be interpreted as a free energy given as a function of the various classical observables in question. If the quantum theory in question is gravitational, then we have reason to expect on physical grounds that there exists a holographic dual in the form of a conformal field theory that has been deformed by operators with sources dual to the boundary conditions of the bulk theory. Thus, if the perturbative expansion of the conformal theory in these sources is an expansion around a free theory with higher spin symmetries, then the sources may correspond to Fronsdal fields in the bulk. Therefore, the existence of such a free energy functional would single out the Vasiliev gauge, as it is required for Fronsdal fields asymptotically. As for the higher spin black holes, it is within this version of the theory that we envisage a possible interpretation of them as gravitational fuzzballs, as discussed in the Introduction. We hope to make this proposal more concrete in a future publication.

As for the $L$-gauge and other gauges that one may surely think of as well, they may describe field configurations on correspondence space geometries that are no longer having the topology of asymptotically anti-de Sitter spacetime (as is the case for the Kruskallike gauge), and/or whose component field descriptions go beyond Fronsdal theory (as for the $L$-gauge, for instance), in the sense discussed above. Moreover, they may admit their proper set of classical observables. It is reasonable to expect that there may also exist suitable action principle and related free energy functionals, lending these solution spaces their physical interpretations. Finally, as for the holomorphic gauge, its physical interpretation may instead be in terms of various deformations of symplectic manifolds. We shall comment on generalized correspondence space geometries and related extensions of Vasiliev's equations towards the end.

Dual boundary conditions in Weyl and normal order. The boundary value formulation that we are trying to implement involves dual boundary conditions, connecting field configurations with finite star product and traces defined in Weyl order with corresponding ones that are real analytic at the origin of twistor space in normal order. A key check is whether the second order correction to the Weyl zero-form can be mapped back to Weyl order and expanded over the assumed class of fiber functions and zero-forms on the base manifold. This is a form of litmus test, since there is not ambiguity in the Vasiliev gauge at leading order, which means that there is no ambiguity in the $Z$-dependent piece of $\widehat{\Phi}^{(2)}$ in the Vasiliev gauge. Upon going to Weyl order, the latter should belong to $L^{1}(\mathcal{Z})$, but it need not be real analytic on $\mathcal{Z}$.

Comparison with quasi-local Fronsdal branch. In a similar fashion, starting from our solutions, it would be very interesting to see whether one could use the gauge freedom to eat up the black hole modes at every order in perturbation theory, while imposing the dual boundary conditions in Weyl and normal order, thereby being left with fully non-linear field configurations expressed only in terms of Fronsdal fields, that could be 
more directly compared with the non-linear results on higher spin gravity derived from holography [109-111].

Alternative perturbative scheme in normal order. We have presented so far a hybrid perturbative approach, combining Weyl and normal order and relying on the trace operation on the extended Weyl algebra. However, there also exists a perturbative approach formulated on-shell entirely within normal order [94] and relying on a different type of (super)trace operations for computing invariants [102]. The main differences between the two approachs are as follows: a) Concerning homotopy integrals, these arise in the hybrid approach in obtaning perturvative expansion for $\widehat{H}$, while they arise in the normal-ordered approach in obtaining the perturbative expansion of the master fields; b) Concerning the treatment of star products of operators in the Fock and anti-Fock spaces, these need to be regularized using the regular prescription following the hybrid approach, whereas it has been proposed in [102] that they are regularized by the homotopy integrals following the normal-ordered approach. We expect that the two approaches lead to master fields that look different as distributions in twistor space, but that they may nonetheless agree at the level of amplitides, as they rely on different trace operations in twistor space.

Comparison of Weyl- and normal-ordered approaches. Our method starts from a factorized internal Ansatz (which is tantamount to starting from total Weyl ordering in twistor space) in order to highlight the generalized projector algebras of special physical meaning that lie at the heart of our construction, and arrives at normal-ordered quantities after taking the star product explicitly, thereby mixing the $Y$ and $Z$ dependence. This procedure can be contrasted with the aforementioned normal-ordered approach to solving Vasiliev's equations in the sector of particle and black hole modes (see for example [3] for the latter), which is already large enough to provide a physically nontrival comparison. The precise relation between these two approaches - which in general also needs to take into account that the gauge-function method introduces other non-polynomial functions of the oscillators from the start - is also related to the problem of determining a proper restriction on the allowed class of functions, gauge transformations and field redefinitions [92-95, 102]. We expect there to be a correspondence between the two approaches only at the level of dual observables, including the on-shell action, but not necessarily at the level of the master fields - similarly to what happens evolving classical fields along either equal time slices or light-fronts.

Generalized higher spin geometries. The higher spin black holes are constructed on correspondence spaces with base manifolds of a definite topology using definite classes of fiber functions given by operators in Fock spaces (the Hilbert space of the harmonic oscillator) including the inner Klein operators introduced via the (non-dynamical) two-form $\widehat{J}$. As we have seen, there is an intricate interplay between the fiber algebra and the singularity structure on the base manifold. This suggests a higher spin landscape based on master field equations containing a dynamical two-form that can be formulated on correspondence spaces of more general topology and with more general noncommutative structure. Dynamical two-forms have been introduced using three-graded internal Frobenius algebras 
in [107], leading to a more general class of Frobenius-Chern-Simons theories [108], that in their turn can be embedded into a broader class of models based on homotopy associative algebras. As for the noncommutative structure of the base manifold, it arises naturally from the quantization of two-dimensional topological sigma models, with target spaces being differential Poisson manifolds that in their turn are special instances of homotopy Poisson manifolds. In particular, in [78] a class of differential Poisson sigma models with extended supersymmetries generated by the Kiling vectors spanning the fibers of the correspondence space has been constructed and proposed as a first quantized description of the Frobenius-Chern-Simons. The fermionic zero-modes of the sigma model generates a closed and central top-form on the fiber space that can be used to provide a more general definition of master fields useful for a globally defined formulation of Frobenius-Chern-Simons theory. We would like to stress that in the landscape of model that we envisage we expect new models whose Weyl zero-forms lend themselves to physical interpretations as density matrices of various quantum mechanical systems obeying nonlinear equtions of motion that one may propose as natural deformations of the von Neumann-Liouville equations derived from the Schrödinger equation. It would be interesting to make this relation clearer and to investigate whether there exists a more general framework for nonlinear quantum mechanics based on underlying topological field theories.

\section{Acknowledgments}

We are grateful to R. Aros, R. Bonezzi, N. Boulanger, D. De Filippi, V.E. Didenko, J. Raeymaekers, E. Sezgin, E.D. Skvortsov, C. Sleight, M. Taronna, M.A. Vasiliev and Y. Yin for stimulating discussions. The research of C. I. was supported by the funding from Grant Agency of Czech Republic, project number P201-12-G028, and by the Russian Science Foundation grant 14-42-00047 in association with the Lebedev Physical Institute in Moscow, that C. I. wishes to thank for kind hospitality at various stages of this work. The work of P. S. is supported by Fondecyt iRegular grant $N^{\circ} 1140296$, Conicyt grant DPI 20140115 and UNAB internal grant DI-1382-16/R.

\section{A Spinor conventions and $\boldsymbol{A d} S_{4}$ background}

We use the conventions of [87] in which $\mathrm{SO}(2,3)$ generators $M_{A B}$ with $A, B=0,1,2,3,0^{\prime}$ obey

$$
\left[M_{A B}, M_{C D}\right]=4 i \eta_{[C \mid[B} M_{A] \mid D]}, \quad\left(M_{A B}\right)^{\dagger}=M_{A B}
$$

which can be decomposed using $\eta_{A B}=\left(\eta_{a b} ;-1\right)$ with $a, b=0,1,2,3$ as

$$
\left[M_{a b}, M_{c d}\right]_{\star}=4 i \eta_{[c \mid[b} M_{a] \mid d]}, \quad\left[M_{a b}, P_{c}\right]_{\star}=2 i \eta_{c[b} P_{a]}, \quad\left[P_{a}, P_{b}\right]_{\star}=i \lambda^{2} M_{a b}
$$

where $M_{a b}$ generate the Lorentz subalgebra $\mathfrak{s o}(1,3)$, and $P_{a}=\lambda M_{0^{\prime} a}$ with $\lambda$ being the inverse $A d S_{4}$ radius related to the cosmological constant via $\Lambda=-3 \lambda^{2}$. Decomposing further under the maximal compact subalgebra, the $A d S_{4}$ energy generator $E=P_{0}=$ 
$\lambda M_{0^{\prime} 0}$ and the spatial $\mathfrak{s o}(3)$ rotations are generated by $M_{r s}$ with $r, s=1,2,3$. In terms of the oscillators $Y_{\underline{\alpha}}=\left(y_{\alpha}, \bar{y}_{\dot{\alpha}}\right)$ defined in (2.15), their realization is taken to be

$$
\begin{gathered}
M_{A B}=-\frac{1}{8}\left(\Gamma_{A B}\right)_{\underline{\alpha \beta}} Y^{\underline{\alpha}} \star Y^{\underline{\beta}}, \\
M_{a b}=-\frac{1}{8}\left[\left(\sigma_{a b}\right)^{\alpha \beta} y_{\alpha} \star y_{\beta}+\left(\bar{\sigma}_{a b}\right)^{\dot{\alpha} \dot{\beta}} \bar{y}_{\dot{\alpha}} \star \bar{y}_{\dot{\beta}}\right], \quad P_{a}=\frac{\lambda}{4}\left(\sigma_{a}\right)^{\alpha \dot{\beta}} y_{\alpha} \star \bar{y}_{\dot{\beta}},
\end{gathered}
$$

using Dirac matrices obeying $\left(\Gamma_{A}\right)_{\underline{\alpha}} \underline{\beta}\left(\Gamma_{B} C\right)_{\underline{\beta \gamma}}=\eta_{A B} C_{\underline{\alpha \gamma}}+\left(\Gamma_{A B} C\right)_{\underline{\alpha \gamma}}$, and van der Waerden symbols obeying

$$
\begin{aligned}
\left(\sigma^{a}\right)_{\alpha}^{\dot{\alpha}}\left(\bar{\sigma}^{b}\right)_{\dot{\alpha}}^{\beta} & =\eta^{a b} \delta_{\alpha}^{\beta}+\left(\sigma^{a b}\right)_{\alpha}{ }^{\beta}, & \left(\bar{\sigma}^{a}\right)_{\dot{\alpha}}{ }^{\alpha}\left(\sigma^{b}\right)_{\alpha}{ }^{\dot{\beta}} & =\eta^{a b} \delta_{\dot{\alpha}}^{\dot{\beta}}+\left(\bar{\sigma}^{a b}\right)_{\dot{\alpha}}^{\dot{\beta}}, \\
\frac{1}{2} \epsilon_{a b c d}\left(\sigma^{c d}\right)_{\alpha \beta} & =i\left(\sigma_{a b}\right)_{\alpha \beta}, & \frac{1}{2} \epsilon_{a b c d}\left(\bar{\sigma}^{c d}\right)_{\dot{\alpha} \dot{\beta}} & =-i\left(\bar{\sigma}_{a b}\right)_{\dot{\alpha} \dot{\beta}}, \\
\left(\left(\sigma^{a}\right)_{\alpha \dot{\beta}}\right)^{\dagger} & =\left(\bar{\sigma}^{a}\right)_{\dot{\alpha} \beta}=\left(\sigma^{a}\right)_{\beta \dot{\alpha}}, & \left(\left(\sigma^{a b}\right)_{\alpha \beta}\right)^{\dagger} & =\left(\bar{\sigma}^{a b}\right)_{\dot{\alpha} \dot{\beta}} .
\end{aligned}
$$

and raising and lowering spinor indices according to the conventions $A^{\alpha}=\epsilon^{\alpha \beta} A_{\beta}$ and $A_{\alpha}=A^{\beta} \epsilon_{\beta \alpha}$ where

$$
\epsilon^{\alpha \beta} \epsilon_{\gamma \delta}=2 \delta_{\gamma \delta}^{\alpha \beta}, \quad \epsilon^{\alpha \beta} \epsilon_{\alpha \gamma}=\delta_{\gamma}^{\beta}, \quad\left(\epsilon_{\alpha \beta}\right)^{\dagger}=\epsilon_{\dot{\alpha} \dot{\beta}} .
$$

The $\mathfrak{s o}(2,3)$-valued connection

$$
\begin{aligned}
\Omega & :=-i\left(\frac{1}{2} \omega^{a b} M_{a b}+e^{a} P_{a}\right) \\
& :=\frac{1}{2 i}\left(\frac{1}{2} \omega^{\alpha \beta} y_{\alpha} \star y_{\beta}+e^{\alpha \dot{\beta}} y_{\alpha} \star \bar{y}_{\dot{\beta}}+\frac{1}{2} \bar{\omega}^{\dot{\alpha} \dot{\beta}} \bar{y}_{\dot{\alpha}} \star \bar{y}_{\dot{\beta}}\right), \\
\omega^{\alpha \beta} & =-\frac{1}{4}\left(\sigma_{a b}\right)^{\alpha \beta} \omega^{a b}, \quad \omega_{a b}=\frac{1}{2}\left(\left(\sigma_{a b}\right)^{\alpha \beta} \omega_{\alpha \beta}+\left(\bar{\sigma}_{a b}\right)^{\dot{\alpha} \dot{\beta}} \bar{\omega}_{\dot{\alpha} \dot{\beta}}\right), \\
e^{\alpha \dot{\alpha}} & =\frac{\lambda}{2}\left(\sigma_{a}\right)^{\alpha \dot{\alpha}} e^{a}, \quad e_{a}=-\lambda^{-1}\left(\sigma_{a}\right)^{\alpha \dot{\alpha}} e_{\alpha \dot{\alpha}},
\end{aligned}
$$

and field strength

$$
\begin{aligned}
\mathcal{R} & :=d \Omega+\Omega \star \Omega \\
& :=\frac{1}{2 i}\left(\frac{1}{2} \mathcal{R}^{\alpha \beta} y_{\alpha} \star y_{\beta}+\mathcal{R}^{\alpha \dot{\beta}} y_{\alpha} \star \bar{y}_{\dot{\beta}}+\frac{1}{2} \overline{\mathcal{R}}^{a b} M_{a b}+\mathcal{R}^{a} P_{a}\right) \\
\mathcal{P}_{\dot{\alpha}}^{\alpha \beta} & =-\frac{1}{4}\left(\sigma_{a b}\right)^{\alpha \beta} \bar{y}_{\dot{\beta}} \mathcal{R}^{a b}, \quad \mathcal{R}_{a b}=\frac{1}{2}\left(\left(\sigma_{a b}\right)^{\alpha \beta} \mathcal{R}_{\alpha \beta}+\left(\bar{\sigma}_{a b}\right)^{\dot{\alpha} \dot{\beta}} \overline{\mathcal{R}}_{\dot{\alpha} \dot{\beta}}\right), \\
\mathcal{R}^{\alpha \dot{\alpha}} & =\frac{\lambda}{2}\left(\sigma_{a}\right)^{\alpha \dot{\alpha}} \mathcal{R}^{a}, \quad \mathcal{R}_{a}=-\lambda^{-1}\left(\sigma_{a}\right)^{\alpha \dot{\alpha}} \mathcal{R}_{\alpha \dot{\alpha}} .
\end{aligned}
$$

In these conventions, it follows that

$$
\begin{aligned}
\mathcal{R}_{\alpha \beta} & =d \omega_{\alpha \beta}-\omega_{\alpha}^{\gamma} \omega_{\gamma \beta}-e_{\alpha}^{\dot{\gamma}} \bar{e}_{\dot{\gamma} \beta}, & & \mathcal{R}_{\alpha \dot{\beta}}=d e_{\alpha \dot{\beta}}+\omega_{\alpha \gamma} \wedge e^{\gamma} \dot{\beta}+\bar{\omega}_{\dot{\beta} \dot{\delta}} \wedge e_{\alpha} \dot{\delta} \\
\mathcal{R}^{a b} & =R_{a b}+\lambda^{2} e^{a} \wedge e^{b}, & & R_{a b}:=d \omega^{a b}+\omega^{a}{ }_{c} \wedge \omega^{c b} \\
\mathcal{R}^{a} & =T^{a}:=d e^{a}+\omega^{a}{ }_{b} \wedge e^{b}, & &
\end{aligned}
$$


where $R_{a b}:=\frac{1}{2} e^{c} e^{d} R_{c d, a b}$ and $T_{a}:=e^{b} e^{c} T_{b c}^{a}$ are the Riemann and torsion two-forms. The metric $g_{\mu \nu}:=e_{\mu}^{a} e_{\nu}^{b} \eta_{a b}$. The $A d S_{4}$ vacuum solution $\Omega_{(0)}=e_{(0)}+\omega_{(0)}$ obeying $d \Omega_{(0)}+\Omega_{(0)} \star \Omega_{(0)}=0$, with Riemann tensor $R_{(0) \mu \nu, \rho \sigma}=-\lambda^{2}\left(g_{(0) \mu \rho} g_{(0) \nu \sigma}-g_{(0) \nu \rho} g_{(0) \mu \sigma}\right)$ and vanishing torsion, can be expressed as $\Omega_{(0)}=L^{-1} \star d L$ where the gauge function $L \in \mathrm{SO}(2,3) / \mathrm{SO}(1,3)$. The stereographic coordinates $x^{\mu}$ of eq. (5.9), are related to the coordinates $X^{A}$ of the five-dimensional embedding space with metric $d s^{2}=d X^{A} d X^{B} \eta_{A B}$, in which $A d S_{4}$ is embedded as the hyperboloid $X^{A} X^{B} \eta_{A B}=-\frac{1}{\lambda^{2}}$, as

$$
x^{\mu}=\frac{X^{\mu}}{1+\sqrt{1+\lambda^{2} X^{\mu} X_{\mu}}}, \quad X^{\mu}=\frac{2 x^{\mu}}{1-\lambda^{2} x^{2}}, \quad \mu=0,1,2,3 .
$$

The global spherical coordinates $(t, r, \theta, \phi)$ in which the metric reads

$$
d s^{2}=-\left(1+\lambda^{2} r^{2}\right) d t^{2}+\frac{d r^{2}}{1+\lambda^{2} r^{2}}+r^{2}\left(d \theta^{2}+\sin ^{2} \theta d \phi^{2}\right),
$$

are related locally to the embedding coordinates by

$$
\begin{aligned}
& X_{0}=\sqrt{\lambda^{-2}+r^{2}} \sin t, \quad X_{0^{\prime}}=\sqrt{\lambda^{-2}+r^{2}} \cos t, \\
& X_{1}=r \sin \theta \cos \phi, \quad X_{2}=r \sin \theta \sin \phi, \quad X_{3}=r \cos \theta \text {, }
\end{aligned}
$$

providing a one-to-one map if $t \in[0,2 \pi), r \in[0, \infty), \theta \in[0, \pi]$ and $\phi \in[0,2 \pi)$ defining the single cover of $A d S_{4}$. This manifold can be covered by two sets of stereographic coordinates, $x_{(i)}^{\mu}, i=N, S$, related by the inversion $x_{N}^{\mu}=-x_{S}^{\mu} /\left(\lambda x_{S}\right)^{2}$ in the overlap region $\lambda^{2}\left(x_{N}\right)^{2}, \lambda^{2}\left(x_{S}\right)^{2}<0$, and the transition function $T_{N}^{S}=\left(L_{N}\right)^{-1} \star L_{S} \in \mathrm{SO}(1,3)$. The map $x^{\mu} \rightarrow-x^{\mu} /(\lambda x)^{2}$ leaves the metric invariant, maps the future and past time-like cones into themselves and exchanges the two space-like regions $0<\lambda^{2} x^{2}<1$ and $\lambda^{2} x^{2}>1$ while leaving the boundary $\lambda^{2} x^{2}=1$ fixed. It follows that the single cover of $A d S_{4}$ is formally covered by taking $x^{\mu} \in \mathbb{R}^{1,3}$.

Petrov's invariant classification of spin-2 Weyl tensors [80-82] is based on their algebraic properties at any spacetime point. Generalized to the higher spin context and by making use of spinor language, it amounts to study the roots of the degree- $2 s$ polynomial $\Omega(\zeta):=C_{\alpha(2 s)} \zeta^{\alpha_{1}} \ldots \zeta^{\alpha_{2 s}}$, where $C_{\alpha(2 s)} \equiv C_{\alpha_{1} \alpha_{2} \ldots \alpha_{2 s}}=C_{\left(\alpha_{1} \alpha_{2} \ldots \alpha_{2 s}\right)}$ is the self-dual part of the Weyl tensor and $\zeta^{\alpha}$ an arbitrary non-vanishing two-component spinor. Factorizing the polynomial in terms of its roots defines a set of $2 s$ spinors which one refers to as principal spinors, viz. $\Omega(\zeta)=u_{\alpha_{1}}^{1} \zeta^{\alpha_{1}} \ldots u_{\alpha_{2 s}}^{2 s} \zeta^{\alpha_{2 s}}$, so $C_{\alpha(2 s)}=u_{\left(\alpha_{1}\right.}^{1} \ldots u_{\alpha_{2 s} s}^{2 s}$. If $\Omega(\zeta)$ has multiple roots, the corresponding principal spinors are collinear. The classification then amounts to distinguish how many different roots $\Omega(\zeta)$ has,i.e., how many non-collinear principal spinors enter the factorization of the spin- $s$ Weyl tensor. Clearly, this classification can be given in terms of the partitions $\left\{p_{1}, \ldots, p_{k}\right\}(k \leq 2 s)$ of $2 s$ in integers obeying $p_{1}+p_{2}+\ldots+p_{k}=2 s$ and $p_{i} \geqslant p_{i+1}$. In the spin-2 case, this singles out the familiar six different possibilities: $\{1,1,1,1\}$ (type I in Petrov's original terminology); $\{2,1,1\}$ (type II); $\{2,2\}$ (type D); $\{3,1\}$ (type III); and $\{4\}$ (type N) plus the trivial case of a vanishing Weyl tensor (type $\mathrm{O})$. The Type D case is related to gravitational field configurations surrounding isolated massive objects; for arbitrary spin-s, we refer to the type $\{s, s\}$ as generalized type $D$. 


\section{B Properties of inner Klein operators}

Working with the chiral integration domain $\mathcal{R}_{\mathbb{R}}$, it makes sense to define the following complex analytic delta functions $\left(M_{\alpha}^{\beta} \in \mathrm{GL}(2 ; \mathbb{C})\right)$ :

$$
\begin{array}{ll}
\delta^{2}(y):=\delta\left(y_{1}\right) \delta\left(y_{2}\right), & \delta^{2}(M y)=\frac{1}{\operatorname{det} M} \delta^{2}(y) \\
\delta^{2}(z):=\delta\left(z_{1}\right) \delta\left(z_{2}\right), & \delta^{2}(M z)=\frac{1}{\operatorname{det} M} \delta^{2}(z) .
\end{array}
$$

Their hermitian conjugates are defined by $\delta^{2}(\bar{y})=\left(\delta^{2}(y)\right)^{\dagger}$ and $\delta^{2}(\bar{z})=\left(\delta^{2}(z)\right)^{\dagger}$. By splitting $y_{\alpha}$ and $z_{\alpha}$ into a complexified Heisenberg algebras

$$
\begin{array}{ll}
{\left[\eta^{-}, \eta^{+}\right]_{\star}=1, \quad \eta^{ \pm}=v^{ \pm \alpha} y_{\alpha}, \quad v^{+\alpha} v_{\alpha}^{-}=-\frac{i}{2},} \\
{\left[\zeta^{-}, \zeta^{+}\right]_{\star}=1, \quad \zeta^{ \pm}=v^{ \pm \alpha} z_{\alpha},}
\end{array}
$$

one can define idempotent inner Kleinian operators

$$
\begin{array}{ll}
\kappa_{y}:=(-1)_{\star}^{N_{y}}, & N_{y}:=\eta^{+} \star \eta^{-}, \\
\kappa_{z}:=(-1)_{\star}^{N_{z}}, & N_{z}:=\zeta^{+} \star \zeta^{-},
\end{array}
$$

using the notation $(c \in \mathbb{C})$

$$
c_{\star}^{\widehat{P}}=\exp _{\star}(\widehat{P} \log c), \quad \exp _{\star} \widehat{P}=\sum_{n=0}^{\infty} \frac{1}{n !} \widehat{P}^{\star n}, \quad \widehat{P}^{\star n}=\underbrace{\widehat{P} \star \cdots \star \widehat{P}}_{n \text { times }},
$$

and representing $(-1)_{\star}^{N_{y}}$ as

$$
(-1)_{\star}^{N_{y}}=\lim _{\epsilon \rightarrow 0} \exp _{\star}\left(i(\pi+\epsilon) N_{y}\right),
$$

idem $(-1)_{\star}^{N_{z}}$. The broken $\operatorname{SL}(2, \mathbb{C})$-invariance is restored in the limit $\epsilon \rightarrow 0$ in Weyl order, viz.

$$
\kappa_{y}=2 \pi \delta^{2}(y), \quad \kappa_{z}=2 \pi \delta^{2}(z) .
$$

We also define

$$
\begin{aligned}
& \bar{\kappa}_{\bar{y}}:=\left(\kappa_{y}\right)^{\dagger}=(-1)_{\star}^{\bar{N}_{\bar{y}}}=2 \pi \delta^{2}(\bar{y}), \\
& \bar{\kappa}_{\bar{z}}:=\left(\kappa_{z}\right)^{\dagger}=(-1)_{\star}^{\bar{N}_{\bar{z}}}=2 \pi \delta^{2}(\bar{z}),
\end{aligned}
$$

using

$$
\begin{aligned}
& \bar{N}_{\bar{y}}:=\left(N_{y}\right)^{\dagger}=\bar{\eta}^{+} \star \bar{\eta}^{-}, \quad \bar{\eta}^{ \pm}:=\left(\eta^{\mp}\right)^{\dagger}=\bar{v}^{ \pm \dot{\alpha}} \bar{y}_{\dot{\alpha}}, \quad \bar{v}_{\dot{\alpha}}^{ \pm}:=\left(v_{\alpha}^{\mp}\right)^{\dagger}, \\
& \bar{N}_{\bar{z}}:=\left(N_{z}\right)^{\dagger}=\bar{\zeta}^{+} \star \bar{\zeta}^{-}, \quad \bar{\zeta}^{ \pm}:=\left(\zeta^{\mp}\right)^{\dagger}=\bar{v}^{ \pm \dot{\alpha}} \bar{z}_{\dot{\alpha}},,
\end{aligned}
$$

such that $\left[\bar{\eta}^{-}, \bar{\eta}^{+}\right]_{\star}=\left[\bar{\zeta}^{-}, \bar{\zeta}^{+}\right]_{\star}=1$. The inner Kleinian elements generate the involutive automorphisms

$$
\begin{array}{ll}
\pi_{y}(\widehat{F}):=\kappa_{y} \star \widehat{F} \star \kappa_{y}, & \pi_{z}(\widehat{F}):=\kappa_{z} \star \widehat{F} \star \kappa_{z}, \\
\bar{\pi}_{\bar{y}}(\widehat{F}):=\bar{\kappa}_{\bar{y}} \star \widehat{F} \star \bar{\kappa}_{\bar{y}}, & \pi_{z}(\widehat{F}):=\bar{\kappa}_{\bar{z}} \star \widehat{F} \star \bar{\kappa}_{\bar{z}},
\end{array}
$$


that act locally on symbols defined in Weyl order, but not on symbols defined in normal order that depends non-trivially on both $Y$ and $Z$. The inner automorphisms $\pi=\pi_{y} \pi_{z}$ and $\bar{\pi}=\bar{\pi}_{\bar{y}} \bar{\pi}_{\bar{z}}$, however, act locally on symbols defined both in Weyl and normal order, viz.

$$
\pi(\widehat{f}(y, \bar{y} ; z, \bar{z}))=\widehat{f}(-y, \bar{y} ;-z, \bar{z}) .
$$

This action is generated by conjugation by the elements

$$
\widehat{\kappa}=\kappa_{y} \star \kappa_{z}, \quad \widehat{\bar{\kappa}}=\kappa_{\bar{y}} \star \kappa_{\bar{z}} .
$$

Their Weyl-ordered symbols can be read off from

$$
\mathcal{O}_{\text {Normal }}(\widehat{\kappa})=\mathcal{O}_{\text {Weyl }}\left((2 \pi)^{2} \delta^{2}(y) \delta^{2}(z)\right), \quad \mathcal{O}_{\text {Normal }}(\widehat{\bar{\kappa}})=\mathcal{O}_{\text {Weyl }}\left((2 \pi)^{2} \delta^{2}(\bar{y}) \delta^{2}(\bar{z})\right),
$$

providing an example of the fact that one and the same operator can be factorized in one order and completely entangled in another order.

\section{Deformed oscillators with delta function Klein operator}

In this appendix, we recall the main steps of the solution of eqs. (3.21)-(3.25), that determine the connection $V_{\alpha}^{\prime}$ on $\mathcal{Z}$ within the Ansatz (3.15)-(3.17). Note that the deformed oscillator problem with a distributional deformation term can be reduced to an ordinary one, with a regular Gaussian source, upon changing ordering prescription, from the Weyl ordering used here to normal ordering on $\mathcal{Z}$. It is then possible to move back the soobtained solution to Weyl ordering and get the same result that we shall review here (see appendix $\mathrm{G}$ in [1] for a detailed proof).

\section{C.1 Problem setting}

As a consequence of the choice (3.15), and of the separation of $Y$ and $Z$ variables in (3.16)(3.17), the deformed oscillators obey

$$
\begin{aligned}
& {\left[\widehat{S}_{\alpha}^{\prime}, \widehat{S}_{\beta}^{\prime}\right]_{\star}=-2 i \epsilon_{\alpha \beta}\left(1-b \Psi \star \kappa_{z}\right),} \\
& {\left[\widehat{\bar{S}}_{\dot{\alpha}}^{\prime}, \widehat{\bar{S}}_{\dot{\beta}}^{\prime}\right]_{\star}=-2 i \epsilon_{\dot{\alpha} \dot{\beta}}\left(1-\bar{b} \bar{\Psi} \star \kappa_{z}\right) .}
\end{aligned}
$$

where the right-hand sides have a distributional deformation term. This problem was solved in [1] (and, in a different gauge, in [3]) for a constant deformation parameter. We can use the same solution method by replacing the constant deformation parameter by $\Psi$, as we shall describe next. 


\section{C.2 Integral equation}

The deformed oscillators $\left(\widehat{S}_{\alpha}^{\prime}, \widehat{\bar{S}}_{\dot{\alpha}}^{\prime}\right)$ can be obtained explicitly by employing the o-product method of [64], later refined in [66] (see also [67]) and adapted to a distributional deformation term in [1]. The method can be articulated in the following two steps:

i) We introduce a spin-frame $u_{\alpha}^{ \pm}$to split $\left(u^{\alpha+} u_{\alpha}^{-}=1\right)$

$$
\widehat{S}_{\alpha}^{\prime}(Y \mid z)=u_{\alpha}^{-} \widehat{S}^{\prime+}(Y, z)-u_{\alpha}^{+} \widehat{S}^{\prime-}(Y, z), \quad\left[\widehat{S}^{\prime-}, \widehat{S}^{\prime+}\right]_{\star}=-2 i\left(1-b \Psi \star \kappa_{z}\right),
$$

and represent $\left(\widehat{S}^{\prime \pm}, \widehat{\bar{S}}^{\prime \pm}\right)$ as $\left(z^{ \pm}:=u^{ \pm \alpha} z_{\alpha}, w_{z}:=z^{+} z^{-},\left[z^{-}, z^{+}\right]_{\star}=-2 i\right)$

$$
\begin{aligned}
\widehat{S}^{\prime \pm} & \equiv u^{ \pm \alpha} \widehat{S}_{\alpha}^{\prime}=4 \int_{-1}^{1} u^{ \pm \alpha} \frac{d t}{(t+1)^{2}} f^{ \pm}(\Psi \mid t) \star z_{\alpha} e^{i \frac{t-1}{t+1} w_{z}} \\
& =4 \int_{-1}^{1} \frac{d t}{(t+1)^{2}} f^{ \pm}(\Psi \mid t) \star z^{ \pm} e^{i \frac{t-1}{t+1} w_{z}} \\
& =-\left.4 i u^{ \pm \alpha} \frac{\partial}{\partial \rho^{\alpha}} \int_{-1}^{1} \frac{d t}{t+1} f^{ \pm}(\Psi \mid t) \star e^{\frac{i}{t+1}\left((t-1) w_{z}+\rho^{\beta} z_{\beta}\right)}\right|_{\rho^{\alpha}=0} \\
& =-\left.4 i \frac{\partial}{\partial \rho_{ \pm}} \int_{-1}^{1} \frac{d t}{t+1} f^{ \pm}(\Psi \mid t) \star e^{\frac{i}{t+1}\left((t-1) w_{z}+\rho^{+} z^{-}-\rho^{-} z^{+}\right)}\right|_{\rho^{ \pm}=0},
\end{aligned}
$$

where $\rho_{\alpha}$ are classical sources, $\epsilon_{\alpha \beta}=u_{\alpha}^{-} u_{\beta}^{+}-u_{\beta}^{-} u_{\alpha}^{+}$and $f^{ \pm}(\Psi \mid t)$ are star-functions of $\Psi$. The virtue of these generalized Laplace transforms is that they are closed under star-product, as can be seen from

$$
\begin{aligned}
& \frac{f^{ \pm}(\Psi \mid t)}{t+1} \star e^{\frac{i}{t+1}\left((t-1) w_{z}+\rho^{+} z^{-}-\rho^{-} z^{+}\right)} \star \frac{f^{ \pm}\left(\Psi \mid t^{\prime}\right)}{t^{\prime}+1} \star e^{\frac{i}{t^{\prime}+1}}\left(\left(t^{\prime}-1\right) w_{z}+\rho^{\prime+} z^{-}-\rho^{-} z^{+}\right) \\
& =\frac{f^{ \pm}(\Psi \mid t) \star f^{ \pm}\left(\Psi \mid t^{\prime}\right)}{2(\tilde{t}+1)} \star e^{\frac{i}{\tilde{t}+1}\left((\tilde{t}-1) w_{z}+\tilde{\rho}^{+} z^{-}-\tilde{\rho}^{-} z^{+}-\frac{i}{2} \rho^{+} \rho^{\prime}+\frac{i}{2} \rho^{-} \rho^{+}\right)} \\
& \tilde{t}:=t t^{\prime}, \quad \tilde{\rho}^{+}:=\rho^{\prime+}+t^{\prime} \rho^{+}, \quad \tilde{\rho}^{-}:=\rho^{-}+t \rho^{\prime-}
\end{aligned}
$$

where the induced map $\left(t, t^{\prime}\right) \mapsto t t^{\prime}$ sends $[-1,1] \times[-1,1]$ to $[-1,1]$, while the $Y$ dependent pieces behave as spectators. In particular,

$$
\begin{aligned}
& {\left[\frac{f^{-}(\Psi \mid t)}{(t+1)^{2}} \star z^{-} e^{i \frac{t-1}{t+1} w_{z}}, \frac{f^{+}\left(\Psi \mid t^{\prime}\right)}{\left(t^{\prime}+1\right)^{2}} \star z^{+} e^{i \frac{t^{\prime}-1}{t^{\prime}+1} w_{z}}\right]_{\star}} \\
& \quad=-\frac{i f^{-}(\Psi \mid t) \star f^{+}\left(\Psi \mid t^{\prime}\right)}{2(\tilde{t}+1)^{2}} \star\left(1+i \frac{\tilde{t}-1}{\tilde{t}+1} w_{z}\right) e^{i \frac{\tilde{t}-1}{\tilde{t}+1} w_{z}} .
\end{aligned}
$$

Thus, upon inserting (C.4) into (C.3), the latter turns into the integral equations

$$
4 \int_{-1}^{1} d t \int_{-1}^{1} d t^{\prime} \frac{f^{-}(\Psi \mid t) \star f^{+}\left(\Psi \mid t^{\prime}\right)}{\left(t t^{\prime}+1\right)^{2}} \star\left[1+i \frac{t t^{\prime}-1}{t t^{\prime}+1} w_{z}\right] e^{i \frac{t t^{\prime}-1}{t t^{\prime}+1} w_{z}}=1-b \Psi \star \kappa_{z},
$$

where we recall that $\kappa_{z}=2 \pi \delta^{2}(z)$; 
ii) Inserting $1=\int_{-1}^{1} d u \delta\left(u-t t^{\prime}\right)\left(t, t^{\prime} \in[-1,1]\right)$ into the left-hand side of (C.9), changing order of integration, and defining

$$
\left(h_{1} \circ h_{2}\right)(\Psi \mid u):=\int_{-1}^{1} d t \int_{-1}^{1} d t^{\prime} h_{1}(\Psi \mid t) \star h_{2}\left(\Psi \mid t^{\prime}\right) \delta\left(u-t t^{\prime}\right),
$$

which is a commutative and associative product ${ }^{27}$ on the space of functions on the unit interval, one arrives at

$$
4 \int_{-1}^{1} \frac{d u}{(u+1)^{2}} h(\Psi \mid u) \star\left[1+i \frac{u-1}{u+1} w_{z}\right] e^{i \frac{u-1}{u+1} w_{z}}=1-2 \pi b \Psi \star \delta^{2}(z),
$$

where $h(\Psi \mid u):=\left(f^{-} \circ f^{+}\right)(\Psi \mid u)$. Next, using the delta function sequence

$$
\lim _{\varepsilon \rightarrow 0} \frac{1}{\varepsilon} e^{-i \sigma \frac{1}{\varepsilon} w_{z}}=\sigma \kappa_{z}
$$

we find the unique solution

$$
h(\Psi \mid t)=\delta(t-1)-\frac{b \Psi}{2} .
$$

Thus, eq. (C.9) is equivalent to the o-product equation

$$
\left(f^{-} \circ f^{+}\right)(\Psi \mid u)=\delta(u-1)-\frac{b \Psi}{2},
$$

with the following solution space, as we shall show in the next subsection:

$$
\begin{aligned}
f^{ \pm} & =g^{\circ( \pm 1)} \circ f, & f(\Psi \mid t) & =\delta(t-1)+j(\Psi \mid t), \\
j(\Psi \mid t) & =q(\Psi \mid t)+\sum_{k=0}^{\infty} \lambda_{k}(\Psi) p_{k}(t), & q(\Psi \mid t) & =-\frac{b \Psi}{4} \star_{1} F_{1}\left[\frac{1}{2} ; 2 ;-\frac{b \Psi}{2} \log t^{2}\right],
\end{aligned}
$$

where $g$ is a gauge artifact (and we use the notation $g^{\circ(+1)}=g$ and $g^{\circ(-1)} \circ g=1$ ); $p_{k}(t):=\frac{(-1)^{k}}{k !} \delta^{(k)}(t)$ act as projectors in the o-product algebra; and $\lambda_{k}$ are given by (C.41) and (C.44).

The presentation (C.12) is compatible with $\kappa_{z} \star f(z)=f(-z) \star \kappa_{z}, \kappa_{z} \star \kappa_{z}=1, \tau\left(\kappa_{z}\right)=-\kappa_{z}$ and $\kappa_{y} \star \kappa_{z}=\widehat{\kappa}$. The fact that $g$ contains gauge artifacts follows by using holomorphic gauge parameters of the form

$$
\epsilon(Y, z)=\int_{-1}^{1} \frac{d t}{1-t^{2}} \breve{\epsilon}(\Psi \mid t) \star e^{i \frac{t-1}{t+1} w_{z}},
$$

which induce

$$
\delta_{\epsilon} f^{ \pm}= \pm \frac{1}{2} \breve{\epsilon} \circ f^{ \pm}
$$

\footnotetext{
${ }^{27}$ For this generalization of the o-product composition to accommodate functions of oscillators, it is of course crucial that the $Y$-dependence comes through one and the same function, $\Psi$ in this case.
} 


\section{C.3 Solution using symbol calculus}

In order to solve eq. (C.14), i.e.

$$
\left(f^{-} \circ f^{+}\right)(\Psi \mid u)=\delta(u-1)-\frac{b \Psi}{2},
$$

one begins by observing that the o-product algebra decomposes into even and odd functions on the interval $[-1,1]$, viz.

$$
f^{(\pi)} \circ g^{\left(\pi^{\prime}\right)}=\delta_{\pi \pi^{\prime}} f^{(\pi)} \circ g^{\left(\pi^{\prime}\right)}, \quad f^{(\pi)}(-s)=\pi f^{(\pi)}(s), \quad \pi, \pi^{\prime}= \pm 1 .
$$

Therefore (C.19) separates into the following two independent equations:

$$
\begin{aligned}
& \left(f^{-(+)} \circ f^{+(+)}\right)(\Psi \mid u)=I_{0}^{(+)}(u)-\frac{b \Psi}{2}, \\
& \left(f^{-(-)} \circ f^{+(-)}\right)(\Psi \mid u)=I_{0}^{(-)}(u),
\end{aligned}
$$

where

$$
I_{0}^{( \pm)}:=\frac{1}{2}[\delta(u-1) \pm \delta(u+1)]
$$

acts as the identity in the even and odd o-product subalgebras. Equations (C.21) and (C.22) can be cast into algebraic equations by expanding $(t \in[-1,1])$

$$
\begin{aligned}
f^{ \pm(\pi)}(\Psi \mid t) & =m^{ \pm(\pi)}(\Psi \mid t)+\sum_{k=0}^{\infty} \lambda_{k}^{ \pm}(\Psi) p_{k}^{(\pi)}(t), \\
m^{ \pm(\pi)}(\Psi \mid t) & =\sum_{k=0}^{\infty} \mu_{k}^{ \pm} I_{k}^{(\pi)}(t) \Psi^{\star k}
\end{aligned}
$$

in terms of $(k \geqslant 1)$

$$
\begin{aligned}
I_{k}^{(\pi)}(u) & :=[\operatorname{sign}(u)]^{\frac{1}{2}(1-\pi)} \int_{-1}^{1} d s_{1} \cdots \int_{-1}^{1} d s_{k} \delta\left(u-s_{1} \cdots s_{k}\right) \\
& =[\operatorname{sign}(u)]^{\frac{1}{2}(1-\pi)} \frac{\left(\log \frac{1}{u^{2}}\right)^{k-1}}{(k-1) !},
\end{aligned}
$$

which obey $(k, l \geqslant 0)$

$$
I_{k}^{(\pi)} \circ I_{l}^{(\pi)}=I_{k+l}^{(\pi)},
$$

and of the o-product projectors $(k \geqslant 0)$

$$
p_{k}^{(\pi)}(s):=\frac{(-1)^{k}}{k !} \delta^{(k)}(s), \quad \pi=(-1)^{k},
$$

obeying

$$
p_{k}^{(\pi)} \circ f=L_{k}[f] p_{k}^{(\pi)}, \quad L_{k}[f](\Psi)=\int_{-1}^{1} d s s^{k} f(\Psi \mid s) .
$$

The property (C.27) implies that upon defining the symbols $(\xi \in \mathbb{C})$

$$
\widetilde{m}^{ \pm(\pi)}(\Psi \mid \xi):=\sum_{k=0}^{\infty} \mu_{k}^{ \pm(\pi)}(\Psi) \xi^{k},
$$


the o-product $m_{-}^{(\pi)} \circ m_{+}^{(\pi)}$ is mapped to the algebraic product $\widetilde{m}_{-}^{(\pi)} \star \widetilde{m}_{+}^{(\pi)}$. Thus, substituting (C.25) into (C.21) and (C.22), and using (C.27) and (C.29), one is left with the algebraic equations

$$
\begin{aligned}
& \widetilde{m}^{-(+)}(\Psi \mid \xi) \star \widetilde{m}^{+(+)}(\Psi \mid \xi)=1-\frac{b \Psi}{2} \xi \\
& \widetilde{m}^{-(-)}(\Psi \mid \xi) \star \widetilde{m}^{+(-)}(\Psi \mid \xi)=1,
\end{aligned}
$$

and the following condition on the coefficients of the projectors in the expansion (C.25):

$$
\lambda_{k}^{-(\pi)} \star L_{n}\left[m^{+(\pi)}\right]+\lambda_{k}^{+(\pi)} \star L_{n}\left[m^{-(\pi)}\right]+\lambda_{k}^{-(\pi)} \star \lambda_{k}^{+(\pi)}=0 .
$$

The solution space to eqs. (C.31) and (C.32) is parameterized by an undetermined function $\widetilde{g}_{\sigma}^{(\pi)}$ as follows: ${ }^{28}$

$$
\widetilde{m}^{ \pm(+)}(\Psi \mid \xi)=\left(\widetilde{g}^{(+)}(\Psi \mid \xi)\right)^{\star( \pm 1)} \star \sqrt{1-\frac{b \Psi}{2} \xi}, \quad \widetilde{m}^{ \pm(-)}(\Psi \mid \xi)=\left(\widetilde{g}^{(-)}(\Psi \mid \xi)\right)^{\star( \pm 1)} .
$$

Likewise, the solution space to (C.33) contains an undetermined set of coefficients, say $\lambda_{k}^{+(\pi)}$. One can show that the undetermined quantities are gauge artifacts. A natural gauge choice is to work with the symmetric solutions

$$
f^{ \pm}=f \quad \Rightarrow \quad \mu_{k}^{ \pm(\pi)}=\mu_{k}^{(\pi)}, \quad \lambda_{k}^{ \pm(\pi)}=\lambda_{k}^{(\pi)} ;
$$

henceforth we shall drop the \pm referring to the spin-frame. Thus

$$
\widetilde{m}^{(+)}(\Psi \mid \xi)=\widetilde{\varepsilon}^{(+)}(\xi) \star \sqrt{1-\frac{b \Psi}{2} \xi}, \quad \widetilde{m}^{(-)}(\Psi \mid \xi)=\widetilde{\varepsilon}^{(-)}(\xi),
$$

where $\left(\widetilde{\varepsilon}^{( \pm)}(\xi)\right)^{\star 2}=1$, and

$$
\lambda_{k}^{(\pi)} \star\left(\lambda_{k}^{(\pi)}+2 L_{k}\left[m^{(\pi)}\right]\right)=0 .
$$

It follows that

$$
\left.m^{(+)}=\varepsilon^{(+)} \circ\left(I_{0}^{(+)}+q^{(+)}\right)\right), \quad m^{(-)}=\varepsilon^{(-)} \circ I_{0}^{(-)},
$$

where $q^{(+)}(\Psi \mid s)$ has the symbol

$$
\widetilde{q}^{(+)}(\Psi \mid \xi)=\sum_{k=1}^{\infty} \mu_{k}^{(\pi)} \xi^{k}=\sqrt{1-\frac{b \Psi}{2} \xi}-1,
$$

corresponding to the confluent hypergeometric function

$$
q^{(+)}(\Psi \mid s)=\sum_{k=1}^{\infty}\left(\begin{array}{c}
\frac{1}{2} \\
k
\end{array}\right)\left(-\frac{b}{2}\right)^{k}(\Psi)^{\star k} \frac{\left(\log \frac{1}{s^{2}}\right)^{k-1}}{(k-1) !}=-\frac{b \Psi}{4} \star{ }_{1} F_{1}\left[\frac{1}{2} ; 2 ; \frac{b \Psi}{2} \log \frac{1}{s^{2}}\right] .
$$

\footnotetext{
${ }^{28}$ Note that, differently from the Lorentz-covariant solutions in $[64,66,67]$, the algebraic equations involve the product of two different functions rather than the square of a single one.
} 
As for the coefficients of the projector $p_{k}$, we have

$$
\lambda_{k}^{(\pi)}=-2 \theta_{k} L_{k}\left[m^{(\pi)}\right], \quad \theta_{k} \in\{0,1\} .
$$

Requiring that $\widehat{S}_{\alpha}^{\prime}=z_{\alpha}$ for $\Psi=0$ and $\theta_{k}=0$, that is

$$
\left.f^{ \pm}(0 \mid s)\right|_{\theta_{k}=0}=\delta(s-1)=I_{0}^{(+)}(s)+I_{0}^{(-)}(s),
$$

implies that

$$
\varepsilon^{( \pm)}=1, \quad \mu_{0}^{(\pi)}=1
$$

From (C.41) and

$$
\begin{gathered}
L_{k}[m]=L_{k}\left[\delta(s-1)+q^{(+)}\right]=1+L_{k}\left[q^{(+)}\right], \\
L_{k}\left[q^{(+)}\right]=-\frac{1+(-1)^{k}}{2}\left[1-\left(1-\frac{b \Psi}{1+k}\right)^{\star 1 / 2}\right],
\end{gathered}
$$

it follows that $\lambda_{k}^{(\pi)}$ are $\Psi$-dependent only for even $k$.

\section{C.4 Non-trivial vacuum connections on $\mathcal{Z}$}

For $\Psi=0$, even though the deformation is absent from (C.1)-(C.2), there still exist nontrivial vacuum solutions, as the coefficients $\lambda_{k \star}$ of o-product projectors $p_{k}$ need not vanish, but rather they reduce to $\left.\lambda_{k \star}\right|_{\Psi=0}=-2 \theta_{k}$, giving rise to a non-trivial flat connection on $\mathcal{Z}$ [1]; see also [67] for similar, $\mathrm{SO}(p, 4-p)$-invariant, non-trivial vacua. In this paper, for simplicity, we shall take $\theta_{k}=0$ for all $k$, deferring the study of the non-trivial vacua to a future work. With this choice, insertion of (C.40) and (C.25) into (C.4) in the symmetric gauge (C.35), yields (3.30).

Open Access. This article is distributed under the terms of the Creative Commons Attribution License (CC-BY 4.0), which permits any use, distribution and reproduction in any medium, provided the original author(s) and source are credited.

\section{References}

[1] C. Iazeolla and P. Sundell, Families of exact solutions to Vasiliev's $4 D$ equations with spherical, cylindrical and biaxial symmetry, JHEP 12 (2011) 084 [arXiv:1107.1217] [INSPIRE].

[2] C. Iazeolla and P. Sundell, Biaxially symmetric solutions to $4 D$ higher-spin gravity, J. Phys. A 46 (2013) 214004 [arXiv:1208.4077] [INSPIRE].

[3] V.E. Didenko and M.A. Vasiliev, Static BPS black hole in 4 d higher-spin gauge theory, Phys. Lett. B 682 (2009) 305 [Erratum ibid. B 722 (2013) 389] [arXiv:0906. 3898] [INSPIRE].

[4] M.A. Vasiliev, Consistent equation for interacting gauge fields of all spins in (3+1)-dimensions, Phys. Lett. B 243 (1990) 378 [INSPIRE].

[5] M.A. Vasiliev, Equations of motion of interacting massless fields of all spins as a free differential algebra, Phys. Lett. B 209 (1988) 491 [INSPIRE]. 
[6] M.A. Vasiliev, Consistent equations for interacting massless fields of all spins in the first order in curvatures, Annals Phys. 190 (1989) 59 [INSPIRE].

[7] M.A. Vasiliev, Properties of equations of motion of interacting gauge fields of all spins in (3+1)-dimensions, Class. Quant. Grav. 8 (1991) 1387 [InSPIRE].

[8] M.A. Vasiliev, More on equations of motion for interacting massless fields of all spins in (3+1)-dimensions, Phys. Lett. B 285 (1992) 225 [INSPIRE].

[9] M.A. Vasiliev, Higher spin gauge theories: Star product and AdS space, hep-th/9910096 [INSPIRE].

[10] M.A. Vasiliev, Higher spin gauge theories in four-dimensions, three-dimensions and two-dimensions, Int. J. Mod. Phys. D 5 (1996) 763 [hep-th/9611024] [INSPIRE].

[11] X. Bekaert, S. Cnockaert, C. Iazeolla and M.A. Vasiliev, Nonlinear higher spin theories in various dimensions, hep-th/0503128 [INSPIRE].

[12] C. Iazeolla, On the algebraic structure of higher-spin field equations and new exact solutions, arXiv:0807.0406 [INSPIRE].

[13] V.E. Didenko and E.D. Skvortsov, Elements of Vasiliev theory, arXiv:1401.2975 [INSPIRE].

[14] R. Rahman and M. Taronna, From higher spins to strings: a primer, arXiv:1512.07932 [INSPIRE].

[15] N. Boulanger and P. Sundell, An action principle for Vasiliev's four-dimensional higher-spin gravity, J. Phys. A 44 (2011) 495402 [arXiv:1102.2219] [INSPIRE].

[16] N. Boulanger, N. Colombo and P. Sundell, A minimal BV action for Vasiliev's four-dimensional higher spin gravity, JHEP 10 (2012) 043 [arXiv:1205.3339] [INSPIRE].

[17] R. Bonezzi, N. Boulanger, E. Sezgin and P. Sundell, An action for matter coupled higher spin gravity in three dimensions, JHEP 05 (2016) 003 [arXiv: 1512.02209] [INSPIRE].

[18] N. Colombo and P. Sundell, Twistor space observables and quasi-amplitudes in $4 D$ higher spin gravity, JHEP 11 (2011) 042 [arXiv:1012.0813] [INSPIRE].

[19] N. Colombo and P. Sundell, Higher spin gravity amplitudes from zero-form charges, arXiv: 1208.3880 [INSPIRE].

[20] M. Flato and C. Fronsdal, One massless particle equals two Dirac singletons: elementary particles in a curved space. 6, Lett. Math. Phys. 2 (1978) 421 [INSPIRE].

[21] B. Sundborg, Stringy gravity, interacting tensionless strings and massless higher spins, Nucl. Phys. Proc. Suppl. 102 (2001) 113 [hep-th/0103247] [INSPIRE].

[22] J. Engquist and P. Sundell, Brane partons and singleton strings, Nucl. Phys. B 752 (2006) 206 [hep-th/0508124] [INSPIRE].

[23] C.-M. Chang, S. Minwalla, T. Sharma and X. Yin, ABJ triality: from higher spin fields to strings, J. Phys. A 46 (2013) 214009 [arXiv:1207.4485] [INSPIRE].

[24] M.R. Gaberdiel and R. Gopakumar, Higher spins \& strings, JHEP 11 (2014) 044 [arXiv: 1406.6103] [INSPIRE].

[25] M.R. Gaberdiel and R. Gopakumar, Stringy symmetries and the higher spin square, J. Phys. A 48 (2015) 185402 [arXiv:1501.07236] [InSPIRE].

[26] M.R. Gaberdiel and R. Gopakumar, String theory as a higher spin theory, JHEP 09 (2016) 085 [arXiv: 1512.07237] [INSPIRE]. 
[27] E. Sezgin and P. Sundell, Massless higher spins and holography, Nucl. Phys. B 644 (2002) 303 [Erratum ibid. B 660 (2003) 403] [hep-th/0205131] [INSPIRE].

[28] I.R. Klebanov and A.M. Polyakov, AdS dual of the critical $O(N)$ vector model, Phys. Lett. B 550 (2002) 213 [hep-th/0210114] [INSPIRE].

[29] E. Sezgin and P. Sundell, Holography in $4 D$ (super) higher spin theories and a test via cubic scalar couplings, JHEP 07 (2005) 044 [hep-th/0305040] [INSPIRE].

[30] S. Giombi and X. Yin, Higher spin gauge theory and holography: the three-point functions, JHEP 09 (2010) 115 [arXiv:0912.3462] [INSPIRE].

[31] S. Giombi and X. Yin, Higher spins in AdS and twistorial holography, JHEP 04 (2011) 086 [arXiv: 1004.3736] [INSPIRE].

[32] S. Giombi, S. Minwalla, S. Prakash, S.P. Trivedi, S.R. Wadia and X. Yin, Chern-Simons theory with vector fermion matter, Eur. Phys. J. C 72 (2012) 2112 [arXiv:1110.4386] [INSPIRE].

[33] M.R. Douglas, L. Mazzucato and S.S. Razamat, Holographic dual of free field theory, Phys. Rev. D 83 (2011) 071701 [arXiv:1011.4926] [InSPIRE].

[34] R. de Mello Koch, A. Jevicki, K. Jin and J.P. Rodrigues, $A d S_{4} / C F T_{3}$ construction from collective fields, Phys. Rev. D 83 (2011) 025006 [arXiv: 1008.0633] [INSPIRE].

[35] D. Das, S.R. Das, A. Jevicki and Q. Ye, Bi-local Construction of $\operatorname{Sp}(2 N) / d S$ Higher Spin Correspondence, JHEP 01 (2013) 107 [arXiv: 1205.5776] [INSPIRE].

[36] D. Anninos, T. Hartman and A. Strominger, Higher spin realization of the $d S / C F T$ correspondence, Class. Quant. Grav. 34 (2017) 015009 [arXiv:1108.5735] [INSPIRE].

[37] M. Henneaux and S.-J. Rey, Nonlinear $W_{\infty}$ as asymptotic symmetry of three-dimensional higher spin anti-de Sitter gravity, JHEP 12 (2010) 007 [arXiv: 1008.4579] [INSPIRE].

[38] A. Campoleoni, S. Fredenhagen, S. Pfenninger and S. Theisen, Asymptotic symmetries of three-dimensional gravity coupled to higher-spin fields, JHEP 11 (2010) 007 [arXiv: 1008.4744] [INSPIRE].

[39] M.R. Gaberdiel and R. Gopakumar, An AdS $S_{3}$ dual for minimal model CFTs, Phys. Rev. D 83 (2011) 066007 [arXiv:1011.2986] [INSPIRE].

[40] M.R. Gaberdiel and T. Hartman, Symmetries of holographic minimal models, JHEP 05 (2011) 031 [arXiv: 1101.2910] [inSPIRE].

[41] M.R. Gaberdiel, R. Gopakumar, W. Li and C. Peng, Higher Spins and Yangian Symmetries, JHEP 04 (2017) 152 [arXiv:1702.05100] [INSPIRE].

[42] M.A. Vasiliev, Holography, unfolding and higher-spin theory, J. Phys. A 46 (2013) 214013 [arXiv:1203.5554] [INSPIRE].

[43] A.K.H. Bengtsson, I. Bengtsson and L. Brink, Cubic interaction terms for arbitrary spin, Nucl. Phys. B 227 (1983) 31 [inSPIRE].

[44] F.A. Berends, G.J.H. Burgers and H. van Dam, On the theoretical problems in constructing interactions involving higher spin massless particles, Nucl. Phys. B 260 (1985) 295 [INSPIRE].

[45] F.A. Berends, G.J.H. Burgers and H. van Dam, Explicit construction of conserved currents for massless fields of arbitrary spin, Nucl. Phys. B 271 (1986) 429 [INSPIRE]. 
[46] E.S. Fradkin and M.A. Vasiliev, On the gravitational interaction of massless higher spin fields, Phys. Lett. B 189 (1987) 89 [INSPIRE].

[47] E.S. Fradkin and M.A. Vasiliev, Cubic interaction in extended theories of massless higher spin fields, Nucl. Phys. B 291 (1987) 141 [inSPIRE].

[48] E.S. Fradkin and R.R. Metsaev, A cubic interaction of totally symmetric massless representations of the Lorentz group in arbitrary dimensions, Class. Quant. Grav. 8 (1991) L89 [INSPIRE].

[49] R.R. Metsaev, Poincaré invariant dynamics of massless higher spins: Fourth order analysis on mass shell, Mod. Phys. Lett. A 6 (1991) 359 [inSPIRE].

[50] R.R. Metsaev, Cubic interaction vertices for fermionic and bosonic arbitrary spin fields, Nucl. Phys. B 859 (2012) 13 [arXiv:0712.3526] [INSPIRE].

[51] R. Manvelyan, K. Mkrtchyan and W. Rühl, General trilinear interaction for arbitrary even higher spin gauge fields, Nucl. Phys. B 836 (2010) 204 [arXiv: 1003.2877] [InSPIRE].

[52] N. Boulanger, S. Leclercq and P. Sundell, On the uniqueness of minimal coupling in higher-spin gauge theory, JHEP 08 (2008) 056 [arXiv:0805.2764] [INSPIRE].

[53] M.A. Vasiliev, Cubic vertices for symmetric higher-spin gauge fields in $(A) d S_{d}$, Nucl. Phys. B 862 (2012) 341 [arXiv:1108.5921] [INSPIRE].

[54] M. Taronna, Higher-spin interactions: four-point functions and beyond, JHEP 04 (2012) 029 [arXiv: 1107.5843] [INSPIRE].

[55] E. Joung, L. Lopez and M. Taronna, Solving the Noether procedure for cubic interactions of higher spins in (A)dS, J. Phys. A 46 (2013) 214020 [arXiv:1207.5520] [INSPIRE].

[56] N. Boulanger, D. Ponomarev and E.D. Skvortsov, Non-abelian cubic vertices for higher-spin fields in anti-de Sitter space, JHEP 05 (2013) 008 [arXiv:1211.6979] [INSPIRE].

[57] E. Joung and M. Taronna, Cubic-interaction-induced deformations of higher-spin symmetries, JHEP 03 (2014) 103 [arXiv:1311.0242] [INSPIRE].

[58] D. Francia, G.L. Monaco and K. Mkrtchyan, Cubic interactions of Maxwell-like higher spins, JHEP 04 (2017) 068 [arXiv: 1611.00292] [INSPIRE].

[59] X. Bekaert, N. Boulanger and P. Sundell, How higher-spin gravity surpasses the spin two barrier: no-go theorems versus yes-go examples, Rev. Mod. Phys. 84 (2012) 987 [arXiv: 1007.0435] [INSPIRE].

[60] S.S. Gubser, I.R. Klebanov and A.M. Polyakov, Gauge theory correlators from noncritical string theory, Phys. Lett. B 428 (1998) 105 [hep-th/9802109] [INSPIRE].

[61] E. Witten, Anti-de Sitter space and holography, Adv. Theor. Math. Phys. 2 (1998) 253 [hep-th/9802150] [INSPIRE].

[62] M. Kontsevich, Deformation quantization of Poisson manifolds. 1, Lett. Math. Phys. 66 (2003) 157 [q-alg/9709040].

[63] V.E. Didenko, A.S. Matveev and M.A. Vasiliev, BTZ black hole as solution of 3D higher spin gauge theory, Theor. Math. Phys. 153 (2007) 1487 [hep-th/0612161] [InSPIRE].

[64] S.F. Prokushkin and M.A. Vasiliev, Higher spin gauge interactions for massive matter fields in 3D AdS space-time, Nucl. Phys. B 545 (1999) 385 [hep-th/9806236] [INSPIRE]. 
[65] P. Kraus and E. Perlmutter, Probing higher spin black holes, JHEP 02 (2013) 096 [arXiv:1209.4937] [INSPIRE].

[66] E. Sezgin and P. Sundell, An exact solution of $4 D$ higher-spin gauge theory, Nucl. Phys. B 762 (2007) 1 [hep-th/0508158] [INSPIRE].

[67] C. Iazeolla, E. Sezgin and P. Sundell, Real forms of complex higher spin field equations and new exact solutions, Nucl. Phys. B 791 (2008) 231 [arXiv:0706.2983] [InSPIRE].

[68] M. Mars, A space-time characterization of the Kerr metric, Class. Quant. Grav. 16 (1999) 2507 [gr-qc/9904070] [inSPIRE].

[69] O. Lunin and S.D. Mathur, AdS/CFT duality and the black hole information paradox, Nucl. Phys. B 623 (2002) 342 [hep-th/0109154] [INSPIRE].

[70] O. Lunin and S.D. Mathur, Statistical interpretation of Bekenstein entropy for systems with a stretched horizon, Phys. Rev. Lett. 88 (2002) 211303 [hep-th/0202072] [INSPIRE].

[71] S.D. Mathur, A proposal to resolve the black hole information paradox, Int. J. Mod. Phys. D 11 (2002) 1537 [hep-th/0205192] [INSPIRE].

[72] S.D. Mathur, The fuzzball proposal for black holes: An elementary review, Fortsch. Phys. 53 (2005) 793 [hep-th/0502050] [INSPIRE].

[73] K. Skenderis and M. Taylor, The fuzzball proposal for black holes, Phys. Rept. 467 (2008) 117 [arXiv: 0804.0552] [INSPIRE].

[74] C.-S. Chu and P.-M. Ho, Poisson algebra of differential forms, Int. J. Mod. Phys. A 12 (1997) 5573 [q-alg/9612031] [INSPIRE].

[75] E.J. Beggs and S. Majid, Semiclassical differential structures, math/0306273 [INSPIRE].

[76] A. Tagliaferro, The star product for differential forms on symplectic manifolds, arXiv: 0809.4717 [INSPIRE].

[77] S. McCurdy and B. Zumino, Covariant star product for exterior differential forms on symplectic manifolds, AIP Conf. Proc. 1200 (2010) 204 [arXiv: 0910.0459] [inSPIRE].

[78] C. Arias, P. Sundell and A. Torres-Gomez, Differential Poisson $\sigma$-models with extended supersymmetry, arXiv:1607.00727 [INSPIRE].

[79] F.A. Berezin and M.A. Shubin, The Schrödinger equation, Moscow University Press, Moscow, (1983).

[80] A.Z. Petrov, The classification of spaces defining gravitational fields, Gen. Rel. Grav. 32 (2000) 1661 [INSPIRE].

[81] R. Penrose and W. Rindler, Spinors and Space-Time, Cambridge University Press, (1986).

[82] R. Penrose and W. Rindler, Spinors And Space-time. Vol. 2: Spinor And Twistor Methods In Space-time Geometry, Cambridge University Press, (1986).

[83] E. Sezgin and P. Sundell, On curvature expansion of higher spin gauge theory, Class. Quant. Grav. 18 (2001) 3241 [hep-th/0012168] [INSPIRE].

[84] E. Sezgin and P. Sundell, Analysis of higher spin field equations in four-dimensions, JHEP 07 (2002) 055 [hep-th/0205132] [INSPIRE].

[85] M.A. Vasiliev, Algebraic aspects of the higher spin problem, Phys. Lett. B 257 (1991) 111 [INSPIRE]. 
[86] K.I. Bolotin and M.A. Vasiliev, Star product and massless free field dynamics in $A d S_{4}$, Phys. Lett. B 479 (2000) 421 [hep-th/0001031] [INSPIRE].

[87] C. Iazeolla and P. Sundell, A Fiber Approach to Harmonic Analysis of Unfolded Higher-Spin Field Equations, JHEP 10 (2008) 022 [arXiv:0806.1942] [INSPIRE].

[88] E. Sezgin and P. Sundell, Geometry and Observables in Vasiliev's Higher Spin Gravity, JHEP 07 (2012) 121 [arXiv:1103.2360] [InSPIRE].

[89] S.S. Gubser and W. Song, An axial gauge ansatz for higher spin theories, JHEP 11 (2014) 036 [arXiv: 1405.7045] [INSPIRE].

[90] J. Bourdier and N. Drukker, On classical solutions of 4 d supersymmetric higher spin theory, JHEP 04 (2015) 097 [arXiv: 1411.7037] [INSPIRE].

[91] P. Sundell and Y. Yin, New classes of bi-axially symmetric solutions to four-dimensional Vasiliev higher spin gravity, JHEP 01 (2017) 043 [arXiv: 1610.03449] [INSPIRE].

[92] N. Boulanger, P. Kessel, E.D. Skvortsov and M. Taronna, Higher spin interactions in four-dimensions: Vasiliev versus Fronsdal, J. Phys. A 49 (2016) 095402 [arXiv: 1508.04139] [INSPIRE].

[93] E.D. Skvortsov and M. Taronna, On locality, holography and unfolding, JHEP 11 (2015) 044 [arXiv: 1508.04764] [INSPIRE].

[94] M.A. Vasiliev, Star-product functions in higher-spin theory and locality, JHEP 06 (2015) 031 [arXiv: 1502.02271] [INSPIRE].

[95] M.A. Vasiliev, Current interactions and holography from the 0-form sector of nonlinear higher-spin equations, arXiv:1605.02662 [INSPIRE].

[96] E. Sezgin, E.D. Skvortsov and Y. Zhu, Chern-Simons matter theories and higher spin gravity, JHEP 07 (2017) 133 [arXiv:1705.03197] [INSPIRE].

[97] V.E. Didenko and M.A. Vasiliev, Test of the local form of higher-spin equations via $A d S / C F T$, arXiv: 1705.03440 [INSPIRE].

[98] M. Taronna, A note on field redefinitions and higher-spin equations, J. Phys. A 50 (2017) 075401 [arXiv: 1607.04718] [INSPIRE].

[99] C. Sleight and M. Taronna, Higher spin gauge theories and bulk locality: a no-go result, arXiv: 1704.07859 [INSPIRE].

[100] P. Breitenlohner and D.Z. Freedman, Stability in gauged extended supergravity, Annals Phys. 144 (1982) 249 [InSPIRE].

[101] C. Iazeolla and J. Raeymaekers, On big crunch solutions in Prokushkin-Vasiliev theory, JHEP 01 (2016) 177 [arXiv: 1510.08835] [INSPIRE].

[102] M.A. Vasiliev, Invariant functionals in higher-spin theory, Nucl. Phys. B 916 (2017) 219 [arXiv: 1504.07289] [INSPIRE].

[103] V.E. Didenko, N.G. Misuna and M.A. Vasiliev, Charges in nonlinear higher-spin theory, JHEP 03 (2017) 164 [arXiv:1512.07626] [INSPIRE].

[104] J. Ambjørn, Y.M. Makeenko, J. Nishimura and R.J. Szabo, Lattice gauge fields and discrete noncommutative Yang-Mills theory, JHEP 05 (2000) 023 [hep-th/0004147] [INSPIRE].

[105] D.J. Gross, A. Hashimoto and N. Itzhaki, Observables of noncommutative gauge theories, Adv. Theor. Math. Phys. 4 (2000) 893 [hep-th/0008075] [INSPIRE]. 
[106] R. Bonezzi, N. Boulanger, D. De Filippi and P. Sundell, Noncommutative Wilson lines in higher-spin theory and correlation functions of conserved currents for free conformal fields, arXiv: 1705.03928 [INSPIRE].

[107] N. Boulanger, E. Sezgin and P. Sundell, $4 D$ higher spin gravity with dynamical two-form as a Frobenius- Chern-Simons gauge theory, arXiv:1505.04957 [INSPIRE].

[108] R. Bonezzi, N. Boulanger, E. Sezgin and P. Sundell, Frobenius-Chern-Simons gauge theory, J. Phys. A 50 (2017) 055401 [arXiv:1607.00726] [INSPIRE].

[109] X. Bekaert, J. Erdmenger, D. Ponomarev and C. Sleight, Towards holographic higher-spin interactions: Four-point functions and higher-spin exchange, JHEP 03 (2015) 170 [arXiv:1412.0016] [INSPIRE].

[110] X. Bekaert, J. Erdmenger, D. Ponomarev and C. Sleight, Quartic AdS interactions in higher-spin gravity from conformal field theory, JHEP 11 (2015) 149 [arXiv:1508.04292] [INSPIRE].

[111] C. Sleight and M. Taronna, Higher spin interactions from conformal field theory: The complete cubic couplings, Phys. Rev. Lett. 116 (2016) 181602 [arXiv:1603.00022] [INSPIRE].

[112] C. Sleight and M. Taronna, Higher-spin algebras, holography and flat space, JHEP 02 (2017) 095 [arXiv: 1609.00991] [INSPIRE].

[113] C. Sleight and M. Taronna, Spinning Witten diagrams, JHEP 06 (2017) 100 [arXiv: 1702.08619] [INSPIRE].

[114] V.E. Didenko, A.S. Matveev and M.A. Vasiliev, Unfolded description of AdS $S_{4}$ Kerr black hole, Phys. Lett. B 665 (2008) 284 [arXiv:0801.2213] [INSPIRE].

[115] V.E. Didenko and E.D. Skvortsov, Exact higher-spin symmetry in CFT: all correlators in unbroken Vasiliev theory, JHEP 04 (2013) 158 [arXiv:1210.7963] [INSPIRE].

[116] M.A. Vasiliev, On the local frame in nonlinear higher-spin equations, arXiv:1707.03735 [INSPIRE].

[117] O.A. Gelfond and M.A. Vasiliev, Operator algebra of free conformal currents via twistors, Nucl. Phys. B 876 (2013) 871 [arXiv:1301.3123] [INSPIRE]. 